University of Louisville

ThinkIR: The University of Louisville's Institutional Repository

\title{
The interaction of homoserine lactones and paraoxonase 2 modulates cell death signaling and cell proliferation.
}

\author{
Aaron Mackallan Neely \\ University of Louisville
}

Follow this and additional works at: https://ir.library.louisville.edu/etd

Part of the Medical Pharmacology Commons, and the Medical Toxicology Commons

\footnotetext{
Recommended Citation

Neely, Aaron Mackallan, "The interaction of homoserine lactones and paraoxonase 2 modulates cell death signaling and cell proliferation." (2017). Electronic Theses and Dissertations. Paper 2735.

https://doi.org/10.18297/etd/2735

This Doctoral Dissertation is brought to you for free and open access by ThinkIR: The University of Louisville's Institutional Repository. It has been accepted for inclusion in Electronic Theses and Dissertations by an authorized administrator of ThinkIR: The University of Louisville's Institutional Repository. This title appears here courtesy of the author, who has retained all other copyrights. For more information, please contact thinkir@louisville.edu.
} 
THE INTERACTION OF HOMOSERINE LACTONES AND PARAOXONASE 2 MODULATES CELL DEATH SIGNALING AND CELL PROLIFERATION

By

Aaron Mackallan Neely

B.S., Paul Quinn College, Dallas, TX, 2007

M.S., University of North Texas, Denton, TX, 2012

M.S., University of Louisville, Louisville, KY, 2016

A Dissertation

Submitted to the Graduate Faculty of the University of Louisville School of Medicine in Partial Fulfillment of the Requirements for the Degree of

Doctor of Philosophy in Pharmacology and Toxicology

Department of Pharmacology and Toxicology

University of Louisville

Louisville, Kentucky

May, 2017 
Copyright 2016 by Aaron Mackallan Neely

All rights reserved 

THE INTERACTION OF HOMOSERINE LACTONES AND PARAOXONASE 2 MODULATES CELL DEATH SIGNALING AND CELL PROLIFERATION

\author{
By
}

Aaron Mackallan Neely

B.S., Paul Quinn College, Dallas, TX, 2007

M.S., University of North Texas, Denton, TX, 2012

M.S., University of Louisville, Louisville, KY, 2016

A Dissertation Approved on

February, 23rd, 2017

by the following Dissertation Committee:

Chi Li, Ph.D.

Leah J. Siskind, Ph.D.

John W. Eaton, Ph.D.

Robert A. Mitchell, Ph.D.

Thomas C. Mitchell, Ph.D. 


\section{DEDICATION}

This dissertation is dedicated to my dear aunt, Sr. Jacinta Neely O.S.B and my dearly departed uncle Alpheus Neely Jr. for their unconditional love, support and encouragement. They taught me how to maximize limited resources to obtain a desired end goal. They encouraged me to transcend beyond socioeconomic limitation and exceed the expectations of others, whether negative or positive. Growing up, Uncle Junior instilled in me a sense of pride and self-worth. Aunt Jacinta showed me that I could be more than my environment allowed and imparted in me valuable lessons such as "Procrastination is the thief of time" and "Your writing is an accurate reflection of who you are". Aunt Jacinta has been a source of spiritual guidance and discipline and Uncle Junior was the only father figure I knew.

To my mother, who was and still is an amazing embodiment of resilience, perseverance and faith! Thank you for your unconditional love, encouragement and support! I love you "mummy"!

To my loved ones who fought bravely and endured the battle against cancer:

Brother Henry Neely O.S.B, Alpheus Neely Jr., Stella Sawyer and Harvey Lester Newton. R.I.P. 


\section{ACKNOWLEDGEMENTS}

Foremost, I would like to thank our Almighty God for giving me this opportunity to further my education and for giving me guidance, favor and good health.

To my mentor Dr. Chi Li, I extend my deepest and sincerest gratitude for his acceptance, tutelage, and endless support. I am appreciative of his guidance, training and encouragement throughout my research. Without his incredible patience, this process would have been an overwhelming undertaking.

I am indebted to Dr. David Hein for his advice, direction and timely wisdom, Dr. Leah Siskind for her support and endless encouragement and Dr. Christopher States for his stern support, for demanding and expecting my absolute best, and his unrelenting confidence in my abilities. Thank you!

My thanks and appreciation is extended to my research committee; Drs. Leah Siskind, John Eaton, Thomas Mitchell and Robert Mitchell, for their enthusiasm, advice and constructive input during this process. Special thanks also go out to, the brothers of Kappa Alpha Psi fraternity Inc. (The Bahamas Alumni Chapter, Leonard Sands, Von Wilson and Dr. Carlton Watson), Cindy and Randy Albury, Lucia Kinteh, Shanice Hudson, Renee' Ettienne, Richard Rankin, Mike Revelett, Brent Gettlefinger, Nicole Phillip-Felix and Denicia Briggs for their support, encouragement and well wishes. I would like to extend thanks to past and 
present members of Chi Li's Laboratory for their continued support and assistance.

Finally, I would like to thank my dear mother (Angela Neely), father (Nathaniel Knowles), aunts (Sr. Jacinta Neely O.S.B and Marie Newton), dearly departed uncle (Alpheus Neely Jr.), sisters (Anastasia Carey and Amanda Johnson) and brother (Adam Johnson) for their unconditional love and support. I would not have been able to complete this dissertation without their continued prayer, love and encouragement. To Mrs. Pam Ferguson and the Ferguson Family, Sr. Cecilia Albury O.S.B, Raja Muhammad and the St. Joseph's Parish Family (Nassau, Bahamas) thank you! 


\section{ABSTRACT}

\section{THE INTERACTION OF HOMOSERINE LACTONES AND PARAOXONASE 2 MODULATES CELL DEATH SIGNALING AND CELL PROLIFERATION}

\section{Aaron Mackallan Neely}

\section{February $23^{\text {rd }}, 2017$}

Pseudomonas aeruginosa produces N-(3-oxododecanoyl)-homoserine lactone (C12) as a quorum-sensing molecule that functions to facilitate bacteria-bacteria communication. $\mathrm{C} 12$ has also been reported to affect many aspects of human host cell physiology, including evoking cell death in various types of cells. However, the signaling pathway(s) leading to $\mathrm{C} 12$-triggerred cell death remains unclear. To clarify cell death signaling induced by $\mathrm{C} 12$, we examined mouse embryonic fibroblasts (MEFs) deficient in one or more caspases. Our data indicate that, unlike most apoptotic inducers, C12 evokes a novel form of apoptosis in cells, probably through the direct induction of mitochondrial membrane permeabilization. Importantly, C12 induces apoptosis in human tumor cells and inhibits tumor growth in animals independent of both pro- and anti-apoptotic $\mathrm{Bcl}-2$ proteins. Previous studies indicate that $\mathrm{C} 12$ requires the 
lactonase/arylesterase paraoxonase 2 (PON2) to exert its cytotoxicity on MEFs. PON2 is known to function as a lactonase to cleave $\mathrm{C} 12$. We found that PON2 was overexpressed in tissues from non-small cell lung carcinoma (NSCLC) patients and oncogenically transformed human bronchia/tracheal epithelial (NHBE) cells. Reducing PON2 expression in NSCLC cell lines as well as several non-transformed cell lines rendered them resistant to C12. However, PON2 expression is only important for the proliferation of NSCLC cells but not that of their untransformed counterparts, indicating that PON2 mediates apoptosis independently of its function to modulate cell proliferation. Overall, our results reveal a unique mitochondrial apoptotic signaling pathway triggered by C12/PON2 interaction and PON2 plays distinct roles in apoptosis signaling and cell proliferation. 


\section{TABLE OF CONTENTS}

PAGE

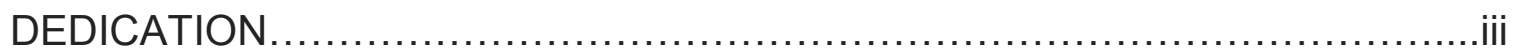

ACKNOWLEDGEMENTS .........................................................

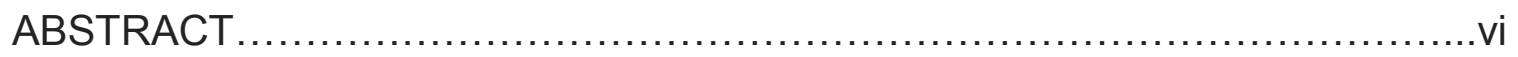

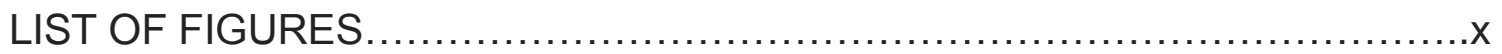

CHAPTER I: INTRODUCTION ............................................

Programmed cell death Apoptosis...........................2

Regulation of Apoptosis...................................

Biological Significance of Apoptosis..........................

The pathways of Apoptosis...............................6

The Intrinsic pathway of apoptosis......................6

The Extrinsic pathway of apoptosis..................10

The Mixed pathway of apoptosis.....................12

Caspases...................................................

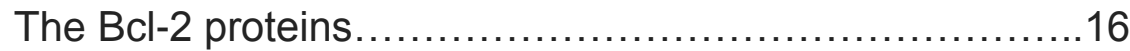

Other cell death modalities-Autophagy and Necrosis.........17

$\mathrm{N}$-(3-oxododecanoyl)-homoserine lactone (C12) ...........18

Paraoxonase 2 (PON2) ...................................20

Hypothesis and Significance of the projects................21 
CHAPTER II: N-(3-OXO-ACYL) HOMOSERINE LACTONE INDUCES APOPTOSIS EXLUSIVLEY THROUGH A MITOCHONDIAL

PATHWAY ......................................................

Introduction....................................................

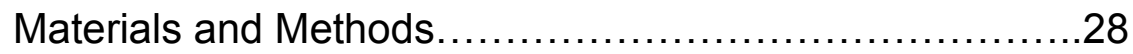

Results................................................ 35

Discussion.....................................................

CHAPTER III: N-(3-OXO-ACYL) HOMOSERINE LACTONE INHIBITS TUMOR GROWTH INDEPENDENT OF BCL-2 PROTEINS................................................. 74

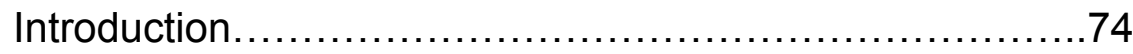

Materials and Methods.....................................77

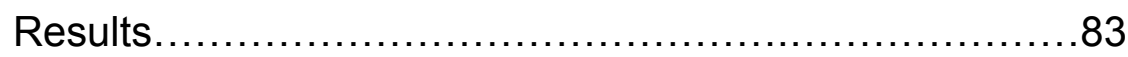

Discussion............................................... 120

CHAPTER IV: PARAOXONASE IS ESSENTAIL FOR THE PROLIFERATIONOF NON-SMALL CELL LUNG CANCER CELLS....................................................123

Introduction ............................................ 123

Materials and Methods....................................125

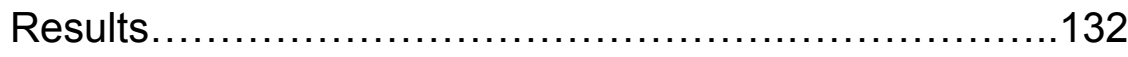

Discussion................................................

CHAPTER V: CONCLUSION AND FUTURE DIRECTIONS..............159

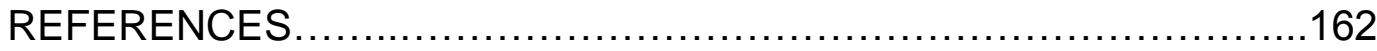

APPENDIX: List of Abbreviations.......................................181

CURRICULUM VITAE ................................................ 


\section{LIST OF FIGURES}

PAGE

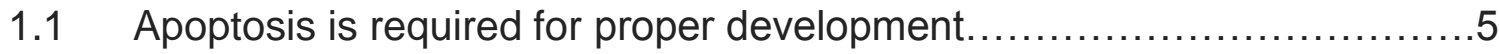

1.2 The intrinsic (mitochondrial) pathway of apoptosis ........................9

1.3 The extrinsic (death receptor) pathway of apoptosis.....................11

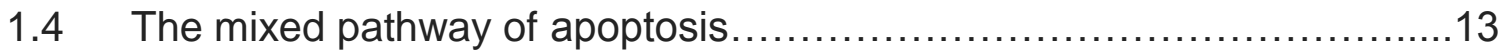

2.1 Caspase-3 and Caspase-7 were required for $\mathrm{C} 12$ induced cell death ....37

2.2 Caspase-3 and Caspase-7 are not required for mitochondrial

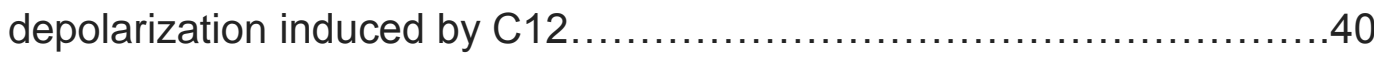

2.3 C12-induced mitochondrial outer membrane permeabilization occurs upstream of caspase $3 / 7$ activation....................................4

2.4 Caspase-8 activation is not involved in C12 induced apoptosis............46

2.5 Caspase-8 activation is not involved in C12-induced tumor cell death....49

2.6 C12 induced mitochondrial membrane potential depolarization is

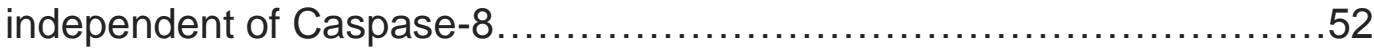

2.7 C12 induced apoptosis is dependent on caspase $-9 \ldots \ldots \ldots \ldots \ldots \ldots \ldots \ldots 5$

2.8 C12 induced mitochondrial potential decrease is independent of

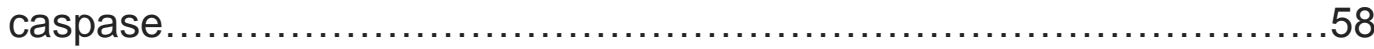

2.9 C12 induced caspase 9 activation occurs downstream of mitochondrial membrane permeabilization...........................................60 
2.10 C12 depolarizes mitochondrial membrane potential without affecting

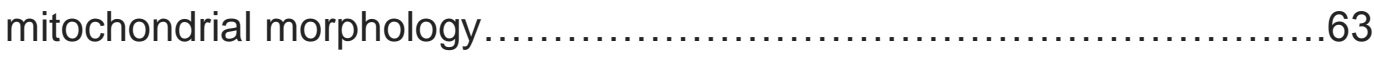

2.11 Lysosomal membrane permeabilization is not involved in cell death

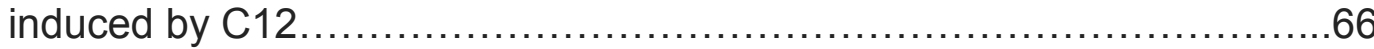

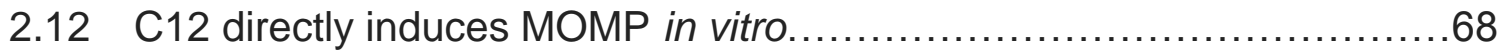

3.1 C12 inhibits LLC tumor growth and induces tumor cell apoptosis in vivo in a dose dependent fashion....

3.2 Administrating $\mathrm{C} 12$ to mice does not affect body weight and organ weight.

3.3 C12 functions synergistically with ABT-737 to induce tumor cell death...92

3.4 C12 induces tumor cell apoptosis independent of anti-apoptotic protein $\mathrm{Bcl}-2$ 96

3.5 Anti-apoptotic $\mathrm{Bcl}-2$ proteins $\mathrm{Mcl}-1$ and $\mathrm{Bcl}-\mathrm{xL}$ do not affect $\mathrm{C} 12$ cytotoxicity. 98

3.6 $\mathrm{C} 12$ induced cytochrome $\mathrm{C}$ release in tumor cells is independent of $\mathrm{Bcl}-$ 2 . 100

3.7 $\mathrm{C} 12$ induced cytochrome $\mathrm{C}$ release in tumor cells is independent of $\mathrm{Mcl}-$ 1 . 102

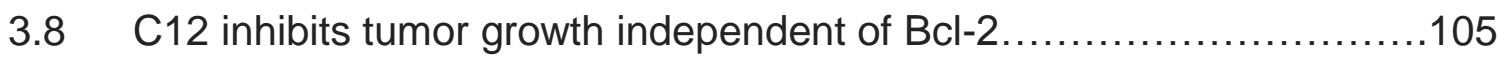

3.9 C12 induced tumor cell apoptosis is independent of Bak or Bax.........108

3.10 Etoposide induces tumor cell death and inhibits xenografted tumor growth dependent of Bak and Bax. 110

3.11 C12 triggered cytochrome $\mathrm{C}$ release in tumor cells is independent of Bak or Bax....

3.12 C12 inhibits xenografted tumor growth independent of Bak and Bax....115

3.13 $\mathrm{C} 12$ induced tumor cell apoptosis is independent of $\mathrm{BH}-3$ only proteins Noxa and Puma.

4.1 PON2 expression is enhanced in human lung tumor tissues and oncogenically transformed HBE cells.

4.2 PON2 blocks chemotherapeutic drug-triggered apoptosis. 136 
4.3 PON2 is required in C12's cytotoxicity on NSCLC cells

4.4 Murine PON2 sensitizes human lung tumor cells with reduced PON2

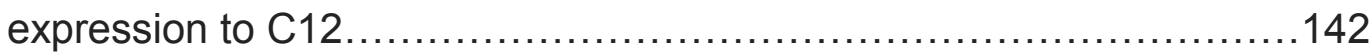

4.5 PON2 is essential for C12-triggered cell death in HEK-293T and HBE

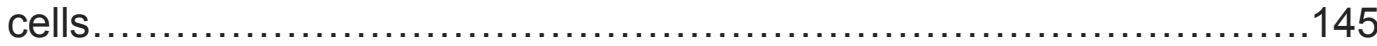

4.6 PON2 is essential for human lung tumor cell proliferation but not nontransformed cell proliferation............................................. 148

4.7 Deficiency in PON2 expression induces G1 cell cycle arrest of A549

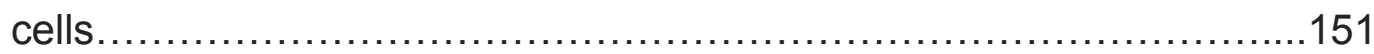

4.8 PON2 interacts with components of the mitochondrial electron transport

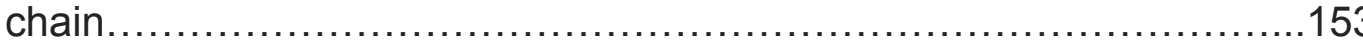




\section{CHAPTER I}

\section{INTRODUCTION}

Tissue differentiation and homeostasis are tightly regulated by the BcL-2 regulated cellular suicide program, apoptosis $(1,2)$. Many human tumor cells acquire resistance to conventional chemotherapeutic drugs that depend on BcL-2 proteins. These neoplastic cells have been found to have an increased ratio of anti-apoptotic to pro-apoptotic Bcl-2 proteins. Thus, the discovery of novel drugs that are capable of overcoming apparent tumor cell BcL-2 protein dependent resistance to cell death is a major challenge. A promising anti-tumor approach is the identification of small molecules (as well as their cellular targets) that preferentially trigger tumor cell apoptosis, independent of tumor cell $\mathrm{Bcl}-2$ protein profile $(3,4)$. While several candidate compounds have been identified, most if not all of these drugs could only induce apoptosis independent of either antiapoptotic or pro-apoptotic Bcl-2 proteins; but not both (4-6). Conversely, the quorum-sensing molecule $\mathrm{N}$-(3-oxododecanoyl)-homoserine lactone (C12) preferentially induces transformed cell apoptosis in vitro and inhibits transplanted tumor growth in vivo independent of both anti- and pro-apoptotic Bcl-2 proteins, thereby making it an ideal candidate drug. Our preliminary data indicates that C12 triggers Bcl-2 protein-independent apoptosis, likely mediated by Paraoxonase 2 (Pon2). PON2 is known to function as a lactonase to cleave $\mathrm{C} 12$. 
Previous studies indicate that $\mathrm{C} 12$ requires the lactonase / arylesterase PON2 to exert its cytotoxicity $(7,8)$

\subsection{Programmed cell death-Apoptosis}

The word Apoptosis derives from Greek origin meaning "dropping off or falling off," and initially referred to the process by which leaves fell from trees or petals from flowers. Apoptosis is a biological phenomenon that consists of a programmed sequence of biochemical events that culminate in the selective elimination of damaged, infected and potentially neoplastic cells from the bodies of multicellular organisms $(9,10)$. Apoptosis is one of the most ubiquitously employed mechanisms by which the body disposes of cell debris or damaged cells without eliciting localized inflammation; due to leakage of cellular contents(11). This mode of cell death is a vital component of normal tissue development, disease progression and maintenance of tissue homeostasis. Additionally, it serves as a means of defense against the development and advancement of cancer (12). The biochemical events that result in the occurrence of Apoptosis also elicit a large number of morphological changes in cells including cell shrinkage, blebbing of the membrane, condensation and fragmentation of nuclear material and the formation of apoptotic bodies.

\subsection{Regulation of apoptosis}

Apoptosis is under stringent genetic control and can be activated by stimuli from multiple sources. It is initiated in response to specific developmental signals or in 
the presence of various stimuli including the reduction of essential growth factors, the activation of Tumor Necrosis Factor receptors (TNFR), DNA damage, loss of cellular attachment, decreases in the local concentration of tissue morphogens and major alterations in homeostatic state of the cell $(13,14)$.

Excess or limited apoptosis can disrupt tissue homeostasis of multicellular organisms. When apoptosis occurs more frequently than cell proliferation does, neurodegenerative disorders are exacerbated. Further to this, the dysregulation of apoptosis has been implicated in the ontogeny and progression of many disease states including many cancers and neurodegenerative disorders such as Alzheimer's, Huntington's and Parkinson's diseases (15-17). Conversely, insufficient apoptosis can precipitate cancer development and progression (18, 19). Thus, tight regulation between apoptosis and cell proliferation is imperative for the viability of all multicellular organisms.

\subsection{Biological significance of apoptosis}

Apoptosis has long been recognized as a critical regulatory component of the development process. The role of apoptosis in development has been investigated and well established in three organisms: Nematodes (Caenorhabditis elegans), Fruit Flies (Drosophila melanogaster) and mouse (Mus musculus). Apoptosis has been found to drive hemaphroditic development during embryogenesis in C. elegans and promote the completion of development in $D$. melanogaster(20). Studies have indicated that the inhibition of apoptosis elicits developmental impediments, disorders and/or death $(12,21)$. 
In early mammalian development, apoptosis is instrumental in the formation of synapses between neurons in the brain and the spinal cord as it facilitates the removal of excess neuron cells (22). Additionally, apoptosis assists in tissue remodeling, molding and shaping of the body and organs and the detailing/separation of extremities (fingers and toes) by the removal of excess tissues. Inhibition or insufficient apoptotic signaling elicits malformed limbs and digits joined by soft tissue (Figure 1.1) (23).
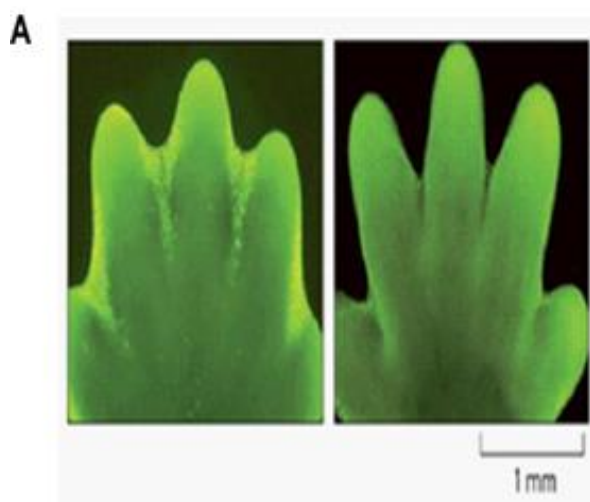

Figure 17-35. Molecular Biology of the Cell, 4th Edition

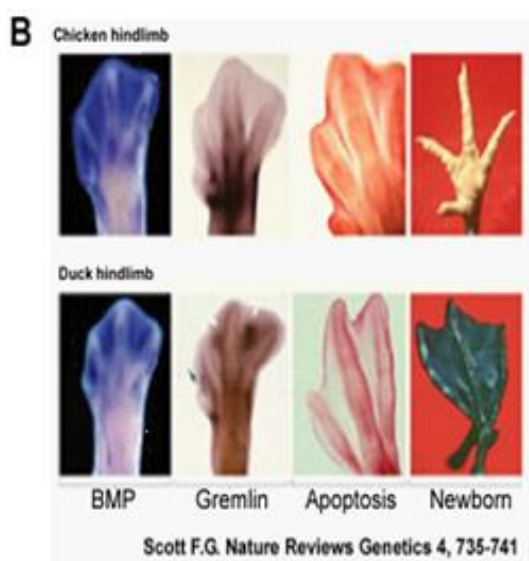


Figure 1.1. Apoptosis is required for proper development. (A) Cells between the digits undergo apoptosis to facilitate proper finger and toe formation. (B) Insufficient apoptosis elicits malformed digits that are joined by soft tissues yielding the persistence of interdigital web as seen in the duck (23). 
Apoptosis is also vital in the removal of those cells that may have deleterious effects on the organism. These include cells that are capable of participating in an autoimmune response as well as virally infected; and thus cytotoxic, T cells (24). Apoptosis facilitates the maintenance of tissue homeostasis. In the average adult, billions of cells die daily via apoptosis and are then replaced with new cells that originate from the body's stem cell populations (25). Apoptosis regulates the constant internal environment and normal tissue homeostasis. Under healthy and normal conditions, the rate of cell proliferation is approximately the same as the rate of apoptosis. This paradigm is essential for ensuring the viability of multicellular organisms. If this balance is disrupted, such that the occurrence of cell death is more frequent than that of cell replacement, the probability of the onset of neurodegenerative disorders is amplified. Moreover, inadequate apoptosis may elicit cancer development (26).

\subsection{The pathways of apoptosis}

Apoptosis typically occurs through one of three signaling pathways, namely the mitochondrial (intrinsic), the death receptor (extrinsic) and the mixed pathways.

\subsubsection{The intrinsic pathway of apoptosis}

Intracellular death signals are translocated to the mitochondria where they induce the mitochondria dependent intrinsic pathway of apoptosis. These signals activate the pro-apoptotic $\mathrm{Bcl}-2$ proteins, which in turn lead to the formation of permeation channels on the outer mitochondrial membrane (OMM). These 
permeation channels facilitate the release of apoptogenic proteins (Diablo/Smac and cytochrome c) from the mitochondrial inter-membrane space (IMS) into the cytosol. Upon release into the cytosol, Diablo/Smac and cytochrome c induce a cascade of caspase reactions that culminate in the occurrence of apoptosis (27). Cytochrome $\mathrm{c}$ activates apoptotic protease factor 1 (apaf1), which promotes the transition of procaspases to their active caspase form. Diablo/Smac promotes apoptosis through direct interaction with inhibitors of apoptosis proteins (IAPs), thereby impeding their ability to inactivate caspases (Figure 1.2) $(28,29)$. 


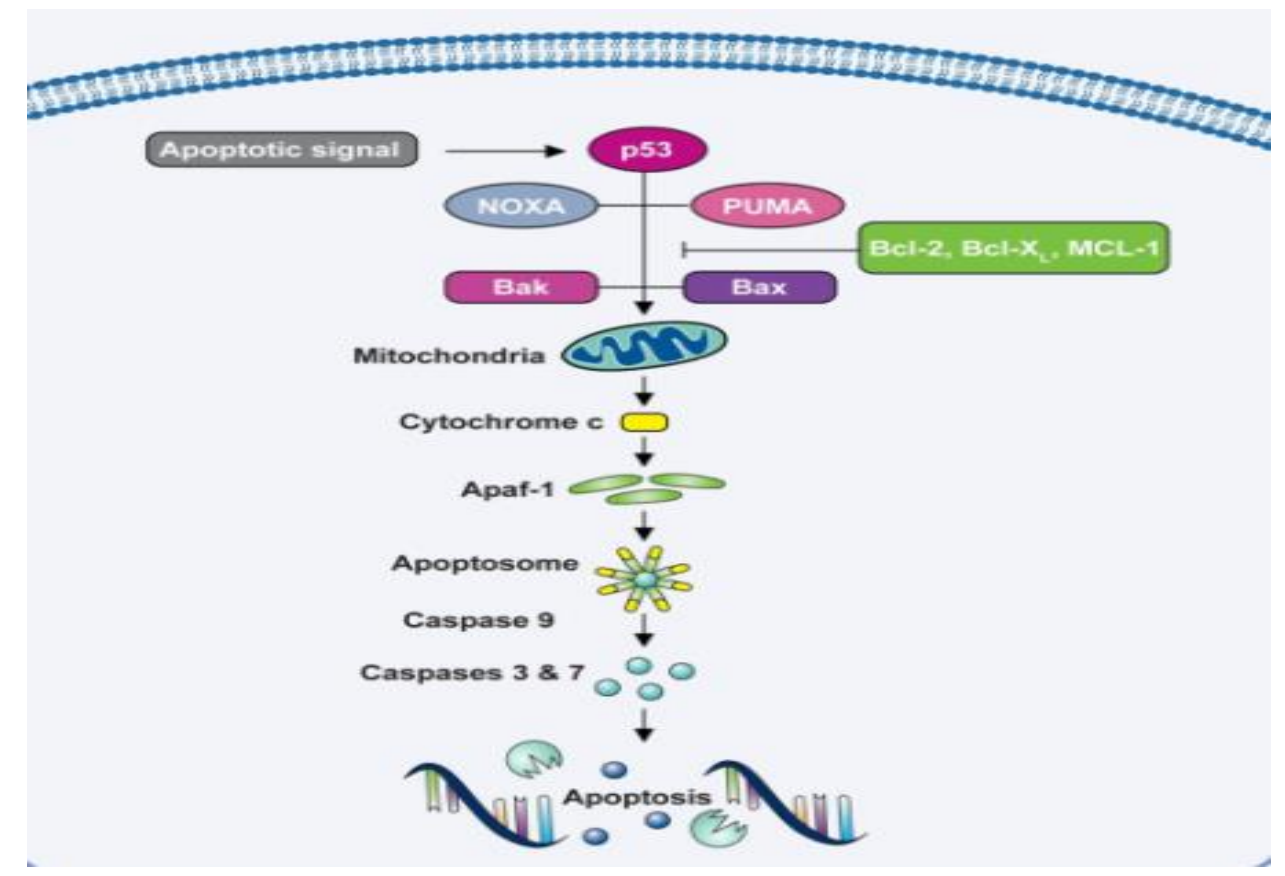


Figure1.2. The intrinsic (mitochondrial) pathway of apoptosis. Apoptotic stimuli; such as DNA damage, elicits p53 activation. Activated p53 activates proapoptotic $\mathrm{Bcl}-2$ proteins which in turn forms permeation channels on the OMM. These permeation channels facilitate the transport of cytochrome $\mathrm{C}$ and Diablo/Smac into the cytosol. While in the cytosol, cytochrome $\mathrm{C}$ initiates a caspase cascade that culminates in the occurrence of apoptosis while Diablo/Smac interacts with IAPs impeding their ability to inhibit caspases thereby promoting apoptosis. The figure is from Dr. Mohd Saquib Khan at Pondicherry University. 


\subsubsection{The extrinsic pathway of apoptosis}

In contrast to the intrinsic apoptotic pathway, death signals for extrinsic apoptotic pathways are initiated from the outside of the cell. Induction of the extrinsic apoptotic pathways does not involve the mitochondria, involvement of the Bcl-2 protein family or the release of cytochrome $\mathrm{c}$ forms the mitochondrial IMS to the cytosol. In this pathway, specific death ligands such as tumor necrosis factor (TNF), Fas Ligand (FasL) or TNF-related apoptosis-inducing ligand (TRAIL) binds to their respective specific transmembrane death receptors including FAS, tumor necrosis factor receptor 1 (TNFR1), p75, DR4(30).

The binding of death ligands to their respective specific death receptor promotes the collection and recruitment of the adaptor protein Fas-associated death domain (FADD) and the inactive forms of the initiator caspases 8 and 10 (procaspases 8 and 10), thus allowing for the formation of the death-inducing signaling complex (DISC). The DISC transports the procaspase molecules in close proximity, thereby ensuring access for their autocatalysis and eventual release into the cytosol (31-35). Caspase 8 or 10 will then activate the effector caspases $3 / 7$ in a cascade precipitated by caspase-mediated reactions that will culminate in the occurrence of apoptosis. 


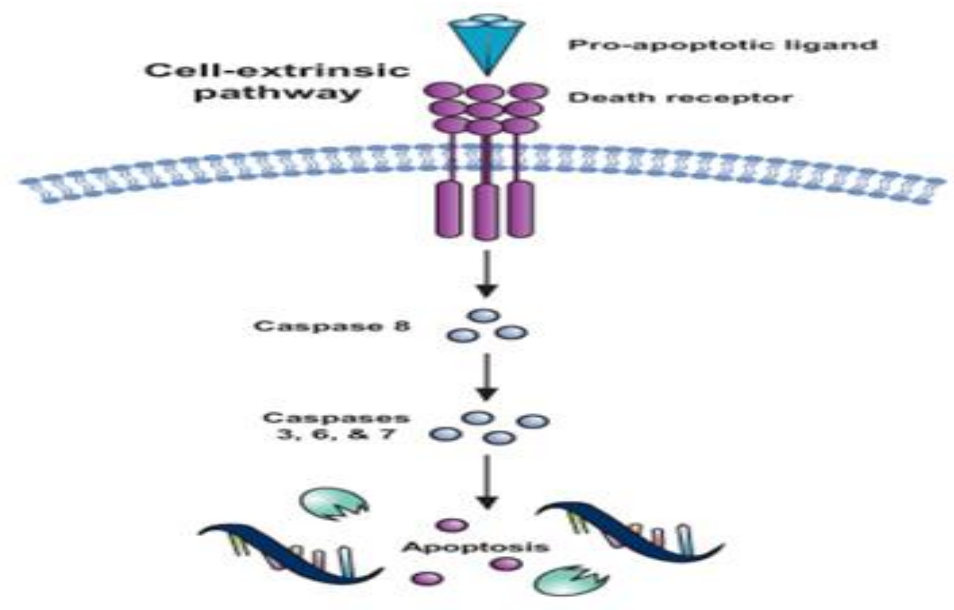

Figure 1.3. The extrinsic (death receptor) pathway of apoptosis. Extrinsic apoptotic signaling is generated extracellularly with the binding of death ligands to specific transmembrane death receptors. Activation of death receptors elicit clustering and recruitment of the adaptor molecule FADD, procaspase 8, 10 resulting in the formation of the DISC. DISC formation activates caspase 8, 10. Caspase 8, 10 then activates caspase $3 / 6 / 7$ and leads to apoptosis. The figure is from Dr. Mohd Saquib Khan at Pondicherry University. 


\subsubsection{The mixed pathway of apoptosis}

There exist certain instances where the induction of apoptotic signaling cascades from external sources can also elicit the activation of both intrinsic and extrinsic apoptotic pathways. This phenomenon is known as the mixed apoptotic pathway and entails death stimuli that are generated extracellularly causing the activation of pro caspase 8 , pro caspase 10 , and formation of the DISC; in the same manner as observed in the extrinsic apoptotic pathway. The DISC formation will ultimately lead to autocatalysis and the eventual activation of caspase 8 and 10 (31-35). When caspase 8 is activated, it can enter one of two routes: (i) it can activate the effector caspases 3, 6 and 7, thereby eliciting apoptosis via the extrinsic pathway or (ii) it can enter the intrinsic apoptotic pathway via interaction with the Bcl-2 protein family. In this instance, caspase 8 will cleave the inactive pro-apoptotic BH3-only protein "Bid" into its truncated and active form tBid. The tBid then activates the Bcl-2 proteins Bax and Bak at the OMM. Bak and Bax then undergo conformational changes as well as oligomerization thereby yielding the formation of permeation pores on the OMM. Permeation pore formation elicits the release of cytochrome $\mathrm{c}$ and Diablo/Smac from the mitochondrial IMS into the cytosol. Once in the cytosol, cytochrome $\mathrm{c}$ and Diablo/Smac exert their proapoptotic effects in the same fashion as they do in the intrinsic apoptotic pathway $(27,36)$. 


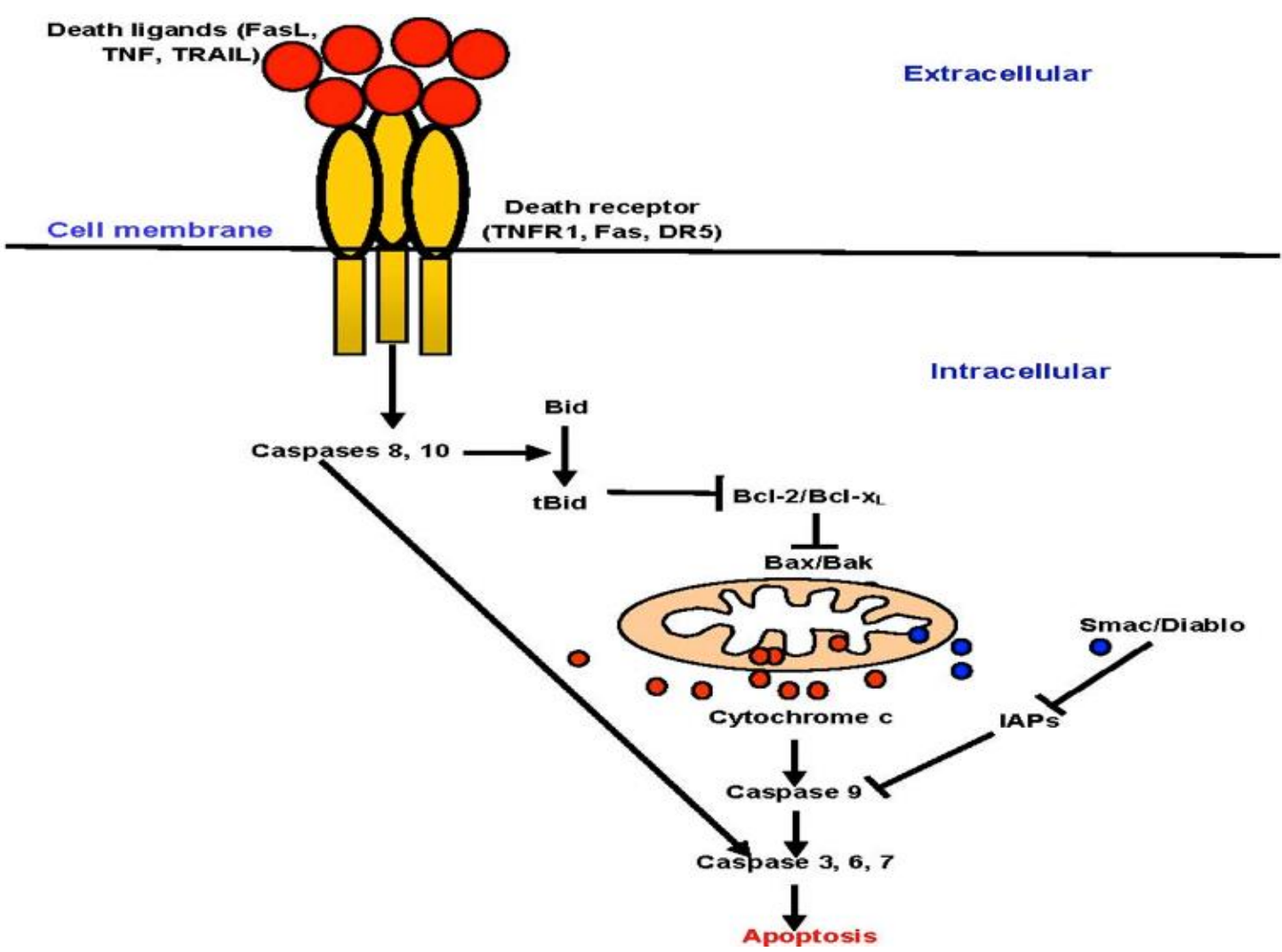


Figure 1.4. The mixed pathway of apoptosis. Extracellular stimuli lead to the formation of DISC and the subsequent activation of caspases 8 and 10. Activated caspase 8 and 10 can cleave effector caspases to elicit cell death via the extrinsic pathway or can enter the intrinsic pathway by interacting with $\mathrm{Bcl}-2$ proteins. Caspase 8 converts Bid into tBid, which then interacts with Bax/Bak inducing their oligomerization and formation of permeation pores on the OMM, which facilitates the transport of apoptogenic proteins such as cytochrome $\mathrm{c}$ and Diablo/Smac out of the mitochondria into the cytosol. 


\subsection{Capases}

The caspases and the $\mathrm{Bcl}-2$ protein family are primary regulators of Apoptosis. The caspases are a group of cysteine proteases, which cleave proteins at sites that are proximal to aspartic acid residues(37). Caspase activation is typically considered the molecular hallmark of apoptosis(38). Caspases exist in their inactive forms (pro caspase), and become activated during apoptosis by proapoptotic proteins(39).

While 12 caspases have been identified to date, not all play a role in the regulation of apoptosis. For instance, caspase 1 is instrumental in the regulation of biological processes that are unrelated to cell death including red blood cell and skeletal muscle myoblast maturation. In contrast, caspase 14 is critical in skin cell development(40).

There are two classes of caspases involved in apoptosis namely the initiator (apical) and the effector (executioner) caspases. The initiator caspases include caspases 2, 8, 9 and 10 and are activated in response to upstream apoptotic stimuli. The initiator caspases cleave and process the effector caspases 3, 6 and 7. Upon activation, the effector caspases trigger cell death by degrading critical intracellular structural proteins. 


\subsection{The $\mathrm{Bcl}-2$ proteins}

The Bcl-2 family of proteins is a major regulator of the intrinsic pathway of apoptosis. Their names derived from B-cell lymphoma/leukemia 2, and are the second member of a collection of proteins initially discovered during chromosomal translocations involving chromosomes 14 and 18 in human follicular lymphomas (41). There are approximately 20 different members of the Bcl-2 protein family. Bcl-2 protein family members all share one to four homologous $\mathrm{Bcl}-2$ homology $(\mathrm{BH})$ domains that are important for homo and hetero-dimeric interactions among different family members. Bcl-2 proteins are either pro-apoptotic or anti-apoptotic $(1,2,13)$ depending on the amount of $\mathrm{BH}$ domains and their ability to regulate apoptosis(42).

Pro-apoptotic $\mathrm{Bcl}-2$ proteins are important for the initiation and stimulation of the intrinsic apoptotic pathway. These proteins are divided into two structurally and functionally distinct categories: multi-domain and BH3-only Bcl-2 proteins(43). Multi-domain pro-apoptotic Bcl-2 proteins Bak, Bax and Bok share three $\mathrm{BH}$ domains (BH1-BH3). They are responsible for the formation of permeation channels on the $\mathrm{OMM}$ that disturbs mitochondrial membrane integrity during apoptosis. This facilitates the release of apoptogenic proteins from the mitochondria into the cytosol (44). Bak and Bax are ubiquitously expressed in all tissues while Bok is present only in reproductive cells (44). Bax and Bak are localized in different subcellular compartments of healthy cells. In the presence of death stimuli Bax undergoes conformational changes at both the amino- and 
carboxyl- termini eliciting its translocation from the cytosol to the $\mathrm{OMM}$ and the eventual formation of large oligomeric complexes. Bak is localized solely in the mitochondria and upon apoptotic stimuli, will undergo conformational changes elicit the formation of oligomeric complexes $(45,46)$. The oligomeric form of Bax and Bak form permeation pores on the OMM (47). These permeation channels mediate the release of pro-apoptotic proteins cytochrome $\mathrm{c}$ and Smac/Diablo from the IMS of the mitochondria to the cytosol where they exert their effects.

\subsection{Other cell death modalities - Autophagy and Necrosis}

Autophagy is a tightly regulated, ordered cell death process. It is self-degradative and essential for the maintenance of the balance of sources of energy at critical stages of development as well as in response to nutrient deprivation(48).

Autophagy is also responsible for the removal of damaged organelles, misfolded and aggregated proteins(49) and the elimination of intracellular pathogens(50).

The deregulation of autophagy has been implicated in non-apoptotic cell death. Autophagy can be selective or non-selective in the removal of specific organelles, ribosomes and protein aggregates(51). Furthermore, autophagy promotes cellular senescence and cell surface antigen presentation. It protects against genomic instability and is critical in the prevention of necrosis(50). Thus, autophagy has a key role in the prevention of diseases including cancer, neurodegeneration, liver diseases, autoimmune diseases, cardiomyopathy and infections. Increased endocytosis, vacuolation, membrane blebbing and nuclear condensation are all characteristic morphological hallmarks of autophagy. This 
type of cell death is categorized as a defensive reaction that can elicit cell death or cell survival(50).

Another modality of cell death is necrosis. Necrosis is the irreversible loss of plasma membrane integrity(52). It is a form of cell injury that results in the premature death of cells in living tissue by autolysis. This type of cell death lacks the features of apoptosis and autophagy, and typically is considered uncontrolled $(52,53)$. Necrosis is typically connected to immoderate cell loss in human pathologies and can lead to local inflammation (54-56), thought to occur through the liberation of factors from dead cells that alert the innate immune system ( 53 , $54,57)$. Necrosis is signaled by irreversible cytoplasmic alterations (condensation, fragmentation and loss of structure) and nuclear changes (pyknosis, karyolysis and karyorhexis) $(52,53)$. Infection, toxins and trauma can result in the unregulated digestion of cell components leading to necrosis.

1.8. $\mathrm{N}$-(3-oxododecanoyl)-homoserine lactone (C12)

The gram-negative opportunistic bacterium Pseudomonas aeruginosa produces $\mathrm{N}$-(3-oxododecanoyl)-homoserine lactone (C12) as a quorum-sensing molecule used to facilitate bacteria-bacteria communication (6). Quorum sensing is a bacterial communication system that releases and detects small diffusible autoinducers $(7,58)$. This system is responsible for the regulation of bacterial gene expression in response to changes in cell population density(58). Gram positive and gram-negative bacteria employ quorum-sensing communication to 
regulate a variety of physiological functions including: symbiosis, antibiotic production, conjugation, motility and biofilm formation(58). Mounting data has shown that $\mathrm{C} 12$ is involved in the regulation of bacterial virulence genes and also interacts with eukaryotic cells $(59,60)$. As a small, lipid-soluble and diffusible molecule, $\mathrm{C} 12$ readily enters cells of multiple tissues in the lungs of cystic fibrosis patients including fibroblasts, epithelial cells, leukocytes, and endothelial cells (8). Additionally C12 alters many aspects of eukaryotic cell physiology including the inhibition of the secretion of proinflammatory cytokines (61-64), activation of p53, and inhibition of events commonly associated with cell death (65-68). C12 has been shown to induce apoptosis in multiple types of cancer cells $(66,69-71)$. C12 induces apoptosis by inhibiting phosphatidylinositide3-kinases, arresting Akt/PKB pathway and attenuating STAT3 activity in breast carcinoma cells (7, 66). In pancreatic carcinoma cells, $\mathrm{C} 12$ induces apoptotic signaling and inhibits cell migration $(7,70)$. In colorectal cancer cells, $\mathrm{C} 12$ reduces the expression of thymidylate synthase while enhancing the activity of otherwise conventional chemotherapeutic agents including 5-fluorouracil (5-FU) (69).

Comparative SAR analysis has indicated that long acyl side chains with a 3-oxo substitution are essential for C12's anti-cancer effect (69). However, the exact signaling pathway(s) leading to C12-triggered cell death remains unclear. Our preliminary studies indicate that $\mathrm{C} 12$-triggered tumor cell apoptosis occurs by selectively activating the mitochondria-specific intrinsic pathway through a novel mechanism that is independent of activities of both anti- and pro-apoptotic Bcl-2 
proteins in human tumor cells. We also find that $\mathrm{C} 12$ induces apoptosis preferentially in oncogenically transformed but not in non-transformed human bronchial epithelial cells. Importantly, we discovered that $\mathrm{C} 12$ cytotoxicity is mediated through the lactonase activity of paraoxonase 2 (PON2).

\subsection{Paraoxonase 2 (PON2)}

PON2 is a ubiquitously expressed mammalian protein with anti-oxidant properties and lactonase/arylesterase activities $(72,73)$. PON2 belongs to the Paraoxonase family of proteins and rapidly hydrolyzes $\mathrm{C} 12$ to $\mathrm{C} 12-$ acid(carboxylic acid), which becomes trapped and accumulates within human bronchial epithelial cells, particularly in mitochondria (74-77). PON2 is upregulated in many types of cancer; including lung cancer, enabling cancer cells to resist conventional therapeutic drugs $(78,79)$. PON2 and PON3 appear to promote chemotherapeutic resistance and cell survival.

PON2 expression also prevents oxidation and inflammation, but the detailed mechanisms remain unclear. This membrane-bound protein's expression is markedly elevated in several human non-small cell lung carcinoma (NSCLC) cell lines. Mutations in the Pon2 gene may be associated with vascular disease and a number of phenotypes related to diabetes.

It has been accepted that oxidative stress derived from the mitochondria, plays an integral role in apoptosis thereby eliciting premature aging and cancer. Recent studies have suggested that various types of cancer take advantage of protection 
derived from increased expression of antioxidant paraoxonase proteins. Microarray studies have suggested an overexpression of PON2 in solid tumors such as hepatocellular carcinomas and prostate carcinomas. Other studies have identified PON2 as a member of a small category of upregulated genes that were correlative with poor prognosis. Studies have shown that PON2 levels are increased in some tumors at the protein level. Investigators observed moderate PON2 overexpression in pancreas, liver, kidney and lung tumors and more than 10-fold upregulation of PON2 in thymus tumors and non-Hodgkins lymphomas. cDNA arrays have shown that PON2 is $\sim 2-4$-fold overexpressed in urinary bladder tumors, liver and kidney tissues in comparison to normal tissues.

\subsection{Hypothesis and Significance of the project}

\subsubsection{Hypothesis and Specific Aims}

N-(3-oxododecanoyl)-homoserine lactone (C12) is produced by Pseudomonas aeruginosa to function as a quorum-sensing molecule for bacteria-bacteria communication. As a small, lipid-soluble, diffusible molecule, $\mathrm{C} 12$ readily enters various types of cells in the lungs of infected patients, including epithelial cells, endothelial cells, fibroblasts and leukocytes, and its concentration is believed to reach high micromolar levels in or adjacent to biofilms formed in the lung airways. C12 has been reported to alter many aspects of human airway epithelial cell physiology, including inhibition of proinflammatory cytokine secretion and activation of events commonly linked with apoptotic cell death. However, the signaling pathway of C12-triggered apoptosis is still unclear. 


\section{Our overall hypothesis is that $\mathrm{C} 12$ triggers a novel form of apoptosis in}

mammalian cells. We proposed the following specific aims to investigate our hypothesis.

Specific aim 1. Investigate the detailed molecular mechanism of apoptosis induced by $\mathrm{C} 12$.

C12 is known to evoke apoptotic cell death in mammalian cells. However, the signaling pathway(s) leading to C12-triggered cell death is (are) still not completely known. Particularly, the potential interplay between extrinsic and intrinsic pathways and "initiator" vs "effector" caspases in C12-activated apoptotic signaling remains unclear. To clarify cell death signaling induced by $\mathrm{C} 12$, we systematically examined mouse embryonic fibroblasts (MEFs) deficient in "initiator" caspases or "effector" caspases.

Specific aim 2. Explore the mechanism of how $\mathrm{C} 12$ induces tumor cell apoptosis and inhibits tumor growth.

C12 has been reported to induce apoptosis in various types of tumor cells. However, the detailed molecular mechanism of $\mathrm{C} 12$-triggerred tumor cell apoptosis is still unclear. In addition, it is completely unknown whether $\mathrm{C} 12$ possesses any potential therapeutic effects in vivo. To gain insights into the mechanism of C12-evoked tumor cell apoptosis, we evaluated the cytotoxic effects of $\mathrm{C} 12$ on tumor cells in vitro, the inhibitory effects of $\mathrm{C} 12$ on tumor 
growth in vivo. In addition, the role of pro- and anti-apoptotic Bcl-2 in C12induced tumor cell apoptosis was systematically examined.

Specific aim 3. Investigate the prospective role of PON2 in C12 cytotoxicity and elucidate the mechanism of PON2 regulation of lung tumor cell proliferation. In nontransformed fibroblast cells, overexpression of Paraoxonase 2 (PON2) promotes cytotoxicity of $\mathrm{C} 12$, but the role of endogenous PON2 in C12-evoked apoptotic signaling was unclear. To further investigate the mechanism of $\mathrm{C} 12$ triggered apoptosis in tumor cells, we studied the involvement of endogenous PON2 in C12 cytotoxicity by stably reducing PON2 expression in human nonsmall cell lung cancer (NSCLC) cells. We also investigated the role of PON2 in proliferation of NSCLC cells.

\subsubsection{Significance of Project}

The data acquired from this project elucidate a novel form of apoptosis induced by $\mathrm{C} 12$ in tumor cells through inducing mitochondrial membrane permeabilization independent of both pro- and anti-apoptotic Bcl-2 proteins. The distinctive proapoptotic feature of $\mathrm{C} 12$ has not been observed in any other apoptosis paradigms, which might be attributed to the ability of $\mathrm{C} 12$ or its metabolite(s) to directly permeabilize mitochondria (within minutes) by forming permeation channels without the involvement of Bcl-2 proteins.

Furthermore, our studies show that expression of PON2 is increased in NSCLC patient tissues, which renders NSCLC cells sensitive to C12 but resistant to 
conventional therapeutic drugs. Importantly, PON2 expression in NSCLC cells is essential for their rapid proliferation. Therefore, $\mathrm{C} 12$ or compounds derived from C12 could trigger rapid and $\mathrm{Bcl}-2$ protein-independent apoptosis in PON2overespressing NSCLC tumors that are resistant to traditional therapeutic drugs, whereas normal tissues are spared due to their lower PON2 expression. Overall, C12 or C12 derivatives may be developed into useful therapies for NSCLC tumors resistant to classical anti-cancer drugs due to dysregulated $\mathrm{Bcl}-2$ protein network and upregulated PON2 expression. 


\section{CHAPTER II}

\section{N-(3-OXO-ACYL)-HOMOSERINE LACTONE INDUCES APOPTOSIS EXCLUSIVELY THROUGH A MITOCHONDRIAL PATHWAY}

\subsection{INTRODUCTION}

The gram-negative aerobic bacterium Pseudomonas aeruginosa is ubiquitously present in soil, water, and vegetation, and is infectious to patients with cystic fibrosis, cancer, burns or compromised immune systems (80). Pseudomonas aeruginosa often infects the lungs of patients with cystic fibrosis, where they form biofilms and eventually become antibiotic resistant (81). Like many other bacteria, Pseudomonas aeruginosa controls these virulence properties by communicating with each other through quorum-sensing, a system of response and stimuli by which the bacteria constitutively generates, releases, detects and responds to small diffusible autoinducers (58). The quorum-sensing molecule $\mathrm{N}$-(3-oxododecanoyl)-L-homoserine lactone (C12) is produced by Pseudomonas aeruginosa and regulates bacterial Lasl/rhll and lasR/rhlR gene networks in addition to influencing bacterial intercellular communication (82, 83). As a small, lipid-soluble and diffusible molecule, $\mathrm{C} 12$ readily enters various types of cells in the lungs of infected patients, including epithelial cells, endothelial cells, fibroblasts and leukocytes $(6,59,84)$, where its concentration is believed to reach high micromolar levels in or adjacent to biofilms formed in the lung airways $(65,85,86)$. C12 has been reported to alter many aspects of 
human airway epithelial cell physiology, including inhibition of proinflammatory cytokine secretion and activation of events commonly linked with apoptotic cell death; including plasma membrane blebbing, cell shrinkage, and nuclear condensation and fragmentation, caspase activation and mitochondrial membrane permeabilization $(65,66,69,87,88)$.

Upon C12 exposure, the activation of diverse signaling pathways associated with apoptosis is observed in different mammalian cells. In breast carcinoma cells, C12 partially inhibits the Akt/PKB pathway, and the JAK/STAT pathway likely mediates pro-apoptotic activities of C12 (66). Similarly, C12 suppresses Akt activation by blocking Akt phosphorylation in undifferentiated intestinal epithelial cells, and overexpression of constitutively active Akt is capable of partially reducing $\mathrm{C} 12$ cytotoxicity (89).

Signaling from the endoplasmic reticulum $(E R)$ has also been implicated in apoptosis caused by C12. ER stress-responsive proteins inositol-requiring enzyme $1 \alpha(\operatorname{IRE} 1 \alpha)$ and X-Box Binding Protein 1 (XBP1) are required for C12induced killing of mouse embryonic fibroblasts (MEF) (90). Furthermore, apoptosis-like morphological changes in the ER and mitochondria are observed in bone marrow-derived macrophages upon C12 treatment (91), and phosphorylation of mitogen-activated protein kinase p38 and eukaryotic translation initiation factor $2 \alpha(\mathrm{elF} 2 \alpha)$ are two biochemical markers indicative of cellular responses to $\mathrm{C} 12(91)$. 
Caspase activation is commonly observed in C12-exposed cells. Caspases are classified into two major categories: "initiator" caspases and "effector" caspases (92), both of which are activated by C12. Serving as a marker for C12-triggered apoptosis, activation of the effector caspase-3 has been detected in airway epithelial cells (65), fibroblasts $(68,93)$, intestinal epithelial cells (89), breast carcinoma cells (66), lymphoma cells (94), lung carcinoma cells (7), colorectal carcinoma cells (95), and leukocytes $(91,96,97)$. Activation of the "initiator" caspases caspase- $8(65,94,97,98)$ and caspase- $9(65,91)$ has also been reported. These data have suggested that both the extrinsic pathway initiated at the plasma membrane and the intrinsic pathway initiated in mitochondria may be activated.

However, the potential interplay between extrinsic and intrinsic pathways and "initiator" vs "effector" caspases in C12-activated apoptotic signaling remain unclear. In this aim, MEFs deficient in one or more caspases were investigated to elucidate the cell death signaling induced by $\mathrm{C} 12$. Our data indicate that $\mathrm{C} 12$ selectively triggers an apoptotic-signaling pathway in which $\mathrm{C} 12$ appears to act by permeabilizing mitochondria, leading to activation of apoptosis. 


\subsection{MATERIALS AND METHODS}

\subsubsection{Reagents}

N-(3-oxododecanoyl)-homoserine lactone (C12), desferrioxamine (DFO), hydrogen peroxide $\left(\mathrm{H}_{2} \mathrm{O}_{2}\right)$, cycloheximide, etoposide and actinomycin D were purchased from Sigma (St. Louis, MO). Propidium iodide (PI) was obtained from Thermo (Waltham, MA). Recombinant human TNF- $\alpha$ was purchased from Peprotech (Rocky Hill, NJ). The Smac mimetic TL-32711 was purchased from Active Biochem (Maplewood, NJ). Unless otherwise stated, all reagents were dissolved in dimethyl sulfoxide (DMSO). Dulbecco's Modified Eagle's Medium (DMEM), penicillin/streptomycin, trypsin, and L-glutamine were obtained from Mediatech (Manassas, VA), and fetal bovine serum (FBS) was purchased from Gemini (Broderick, CA). Caspase-Glo assay 3/7 kit was purchased from Promega (Madison, WI). Antibodies (Abs) used for western blot analysis were anti- $\beta$-actin mAb (Sigma), anti-full-length-caspase-3 pAb; anti-full-lengthcaspase-7 pAb; anti- full-length-caspase-8 pAb; anti- full-length-caspase-9 pAb; anti-cleaved-caspase-3 pAb; anti-cleaved-caspase-7 pAb; anti-PARP pAb (Cell signaling; Danvers, MA),), anti-cytochrome c mAb for western blot (Santa Cruz; Dallas, TX), anti-cytochrome c mAb for immunofluorescence staining (BD Transduction Laboratory; San Jose, CA), anti-Tom40 pAb (Santa Cruz), antiTom20 pAb (a gift from Dr. Brian Wattenberg), peroxidase-conjugated goat antirabbit IgG (Thermo) and peroxidase-conjugated goat anti-mouse IgG (Thermo). 


\subsubsection{Cell lines and cell culture}

Immortalized mouse embryonic fibroblasts (MEFs) deficient in the expression of caspase-8 and their wild-type counterparts were provided by Professor David Vaux (Walter and Eliza Hall Institute of Medical Research, Parkville, VIC Australia). MEFs lacking caspase-9 and their wild-type counterparts were obtained from Professor Jerry Adams (Walter and Eliza Hall Institute of Medical Research). MEFs lacking caspase-3, caspase-7, caspase-3 and caspase-7, or their wild-type counterparts were obtained from Professor Richard Flavell (Yale University). Human colorectal carcinoma HCT116 and human pancreatic carcinoma Panc-1 were purchased from ATCC (Manassas, VA). To generate HCT116 and Panc-1 cells with reduced caspase-8 expression or vector control, cells were first infected by caspase-8 shRNA lentiviral particles or control shRNA lentiviral particles with $10 \mu \mathrm{g} / \mathrm{ml}$ polybrene (Santa Cruz). Stable cells were obtained by culturing cells in the medium with $5 \mu \mathrm{g} / \mathrm{ml}$ puromycin. All of the cell lines were grown and maintained in DMEM supplemented with $10 \%$ FBS, 100 units $/ \mathrm{ml}$ penicillin, and $100 \mu \mathrm{g} / \mathrm{ml}$ streptomycin. Cells were all cultured in a $5 \% \mathrm{CO}_{2}$ humidified incubator at $37^{\circ} \mathrm{C}$.

\subsubsection{Cell Viability/Death Assays}

MEF cell lines were plated in a 48-well tissue culture plate with 20,000 cells in each well and cultured for 24 hours. Following treatment with different concentrations of $\mathrm{C} 12$, cells were harvested in the presence of $1.0 \mu \mathrm{g} / \mathrm{ml}$ propidium iodide $(\mathrm{PI})$. Cell viability was measured by $\mathrm{PI}$ exclusion using flow 
cytometry (FACScalibur, Beckon Dickinson; San Jose, CA). The percentage of cell death was determined as 100 minus the cell viability measurement.

\subsubsection{Caspase-3/7 activity}

Caspase-3/7 activities were measured using a Caspase-Glo assay kit (Promega, Madison, WI)(59). In this assay, the proluminescent substrate containing the amino acid sequence Asp-Glu-Val-Asp (DEVD) is cleaved by activated caspase-3/7, resulting in the release of a luciferase substrate (aminoluciferin) and the production of luminescent signal. 24 hours before the treatment, cells were plated in white-walled 96-well plates. At the indicated time points following treatment with various agents, cells were mixed with CellTiterGlo reagent, and luminescence was quantified by a Gemini EM microplate spectrofluorometer (Molecular Devices; Sunnyvale, CA) according to the manufacturer's protocol. Data were presented as relative luminescence units (RLUs).

\subsubsection{Western blot analysis}

Equal amounts of proteins $(30 \mu \mathrm{g})$ were separated on a $4-12 \%$ Bis-Tris gel (BioRad; Hercules, CA) and transferred onto PVDF membrane (Millipore; Billerica, MA). The membrane was incubated with appropriate primary or secondary antibodies either overnight at $4^{\circ} \mathrm{C}$ or at room temperature for 3 hours in $1 \mathrm{X}$ phosphate-buffered saline (PBS) containing 5\% (w/v) nonfat dry milk (Bio-Rad) and $0.2 \%(\mathrm{v} / \mathrm{v})$ Tween 20. Protein levels were detected using the enhanced 
chemiluminescent detection system (Pierce; Rockford, IL) as described previously (99).

2.2.6. Measuring $\Delta \psi$ mito using imaging microscopy of $\mathrm{JC} 1$

For imaging experiments to measure mitochondrial membrane potential ( $\Delta \psi$ mito), cells were incubated with growth media containing the $\Delta \psi$ mito probe $\mathrm{JC} 1(10 \mu \mathrm{M})$ for 10 minutes at room temperature, and then washed three times with Ringer's solution to remove the extra dye. JC1-loaded cells were placed onto a chamber on the stage of a Nikon Diaphot inverted microscope. Cells were maintained at room temperature during the course of the experiments. Treatments were made by diluting stock solutions into Ringer's solution at the concentrations stated in the text. Fluorescence imaging evaluation of $\Delta \psi$ mito was carried out using equipment and methods that have been reported previously $(93,95)$. Briefly, a Nikon Diaphot inverted microscope with a Fluor $20 \mathrm{X}$ objective ( 0.75 numerical aperture) was used. A charge coupled device camera acquired JC-1 emission images (green: 510-540 nm; red: 580-620 nm) during excitation at $490+/-5 \mathrm{~nm}$ using filter wheels (Lambda-10, Sutter Instruments, Novato, CA). Axon Imaging Workbench 5.1 (Axon Instruments, Foster City, CA) controlled filters and collection of data. Images were corrected for background using regions without cells. The data were calculated with JC1 fluorescence ratios normalized to minimal $\mathrm{JC} 1$ ratios obtained at the beginning of the experiment and maximal JC1 ratios acquired following $5 \mu \mathrm{M} \mathrm{FCCP}$ treatment. Normalized JC1 Ratio $=($ Ratio at a given time - minimal Ratio $) /$ 
(maximal ratio - minimal Ratio) ${ }^{*} 100 \%$. Normalized JC1 ratios at plateau (before FCCP treatment) from at least three different experiments have been averaged for summary graphs.

\subsubsection{Immunofluorescence microscopy}

MEFs plated onto cover glasses 24 hours earlier were rinsed with Ringer's solution and incubated for 4 hours with either vehicle (DMSO) or $50 \mu \mathrm{M} \mathrm{C12} \mathrm{in}$ Ringer's solution. The immunofluorescence staining of cytochrome $\mathrm{c}$ and Tom20 were carried out as described previously (95). Images were captured using a Nikon Eclipse Ti confocal microscope (Nikon; Melville, NY) equipped with a PlanApo 60x, 1.42 NA oil immersion objective. To minimize variability for quantitative assessment, the same microscope settings were used across vehicle control and C12-treated samples for three individual experiments. Four to nine fields of view were captured to acquire a sample size of at least 100 cells for each individual experiment. Intracellular distribution of cytochrome c and TOM20 was analyzed using ImageJ $(\mathrm{NIH})$. Cytochrome c release from mitochondria was quantified by determining the standard deviation (SD) of the mean pixel intensity of cytochrome $c$ of each cell - this is named as the punctate/diffuse index (100-102). Briefly, when cytochrome $\mathrm{c}$ is highly localized within mitochondria, it will display a punctate distribution pattern, leading to high SD of the average pixel intensity, but when cytochrome $\mathrm{c}$ is distributed throughout the cell, the SD will be low. Therefore, a decrease in the SD 
(punctate/diffuse index) is an index of cytochrome c redistribution from mitochondria to the cytosol.

\subsubsection{Mitochondrial morphology}

To image mitochondrial morphology and membrane potential using confocal microscopy, cells were transfected with mitochondrial matrix-targeted GFP plasmid (Clontech; Madison, WI) using an Amaxa nucleofector device (Lonza; Allendale, NJ), seeded onto $35 \mathrm{~mm}$ glass coverslips and placed in a tissue culture incubator. After 16-24 hours coverslips were mounted in a recording chamber positioned on the stage of an inverted microscope (IX71; Olympus America Inc., Center Valley, PA). Cells were visualized using a PlanApo 60x, 1.42 NA oil immersion objective and confocal time series images acquired using a VT-Infinity 3 (VisiTech International; Sunderland, UK). The chamber was perfused with Hank's Balanced Salt Solution containing $20 \mathrm{nM}$ mitochondrial membrane potential probe tetramethylrhodamine, ethyl ester (TMRE) at room temperature. After a 15 minute equilibration period, GFP and TMRE were alternately excited using the 488 and $568 \mathrm{~nm}$ lines respectively of a KryponArgon laser. The emitted fluorescence was filtered using a dual bandpass filter set (VisiTech International) and collected and using HCImage software (Hamamatsu Corporation; Sewickley, PA). Mitochondrial morphology was analyzed using ImageJ to determine the mean area/perimeter ratio and inverse circularity as the measurement of interconnectivity and elongation respectively, as described previously. 
2.2.9. Detection of the release of cytochrome $\mathrm{c}$ from mitochondria Mitochondria were purified from MEFs as described previously (25). Isolated mitochondria were resuspended in buffer containing 12 mM HEPES $(\mathrm{pH} 7.5)$, $1.7 \mathrm{mM}$ Tris- $\mathrm{HCl}$ (pH 7.5), $100 \mathrm{mM} \mathrm{KCl,} 140 \mathrm{mM}$ mannitol, $23 \mathrm{mM}$ sucrose, 2 $\mathrm{mM} \mathrm{KH} \mathrm{PO}_{4}, 1 \mathrm{mM} \mathrm{MgCl}$, $0.67 \mathrm{mM}$ EGTA, and $0.6 \mathrm{mM}$ EDTA supplemented with protease inhibitors (Complete; Roche Diagnostics, Indianapolis, IN). After one hour incubation with $\mathrm{C} 12$ at $30^{\circ} \mathrm{C}$, mitochondrial vesicles were centrifuged at $10,000 \times \mathrm{g}$ for $10 \mathrm{~min}$ and then dissolved in $1 \times$ SDS-PAGE loading buffer. Proteins in the supernatant and pellet fractions were detected by Western blotting.

2.2.10. Statistical analysis

All experiments were performed in triplicate at least three times. Results are presented as mean \pm standard deviation. Statistical analysis was performed using Student's two tail t-test. A p value $<0.05$ was considered significant. 


\subsection{RESULTS}

\subsubsection{Apoptosis is the major form of cell death caused by $\mathrm{C} 12$.}

In multicellular organisms, cell death is a highly heterogeneous process in which several distinct, in some cases partially overlapping, cell signaling cascades can be activated (103). Although C12's ability to trigger the events commonly linked to apoptosis has been reported $(65,66,90,93,96)$, it is unclear whether other cell death signaling is also involved. Caspase-3 and caspase-7 are two highly related "effector" caspases, and mouse embryonic fibroblasts (MEFs) deficient in expression of both caspases are markedly resistant to both mitochondrial and death receptor-mediated apoptosis (104). To further characterize $\mathrm{C} 12$-induced signaling leading to cell death, we first investigated whether caspase- 3 and caspase- 7 were essential to mediate cytotoxic effects of $\mathrm{C} 12$. Cytotoxicity of $\mathrm{C} 12$ was examined in MEFs lacking caspase-3 (caspase-3-KO), caspase-7 (caspase-7-KO), or both (caspase-3/7DKO) as well as their wild-type (WT) counterparts (Figure 2.1A). C12 induced significant cell death in WT, caspase-3-KO and caspase-7-KO MEFs, whereas caspase-3/7-DKO MEFs were completely resistant to C12 exposure (Figure 2.1B). Moreover, less cell death was detected in caspase-3-KO or caspase-7KO MEFs than their wild-type counterparts (Figure 2.1B). These data indicate that both caspase- 3 and caspase- 7 are involved in apoptosis initiated by $\mathrm{C} 12$; caspase-3 appeared to play a more prominent role than caspase-7. Importantly, cleavage of caspase-3 and caspase-7, one of the hallmarks of apoptosis, was detected in WT MEFs treated with C12 (Figure 2.1C). Furthermore, a known substrate of caspase-3 and caspase-7 PARP was also 
cleaved in WT MEFs upon C12 exposure, but not in their caspase-3/7-DKO counterparts. Overall, the essential role of caspase- 3 and caspase- 7 indicates that cell death induced by $\mathrm{C} 12$ is largely attributed to apoptosis. 
A

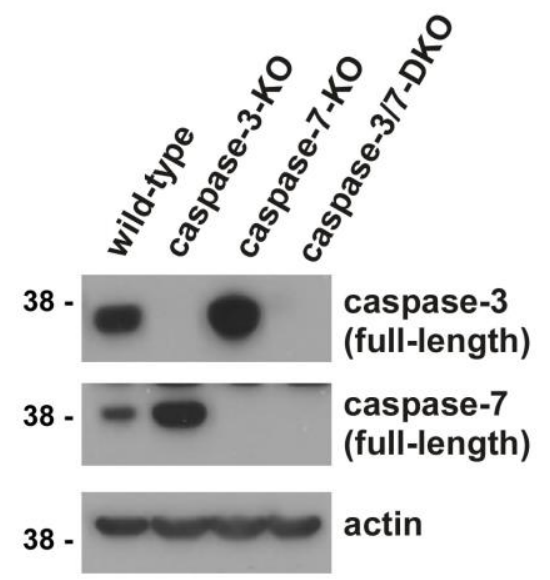

B

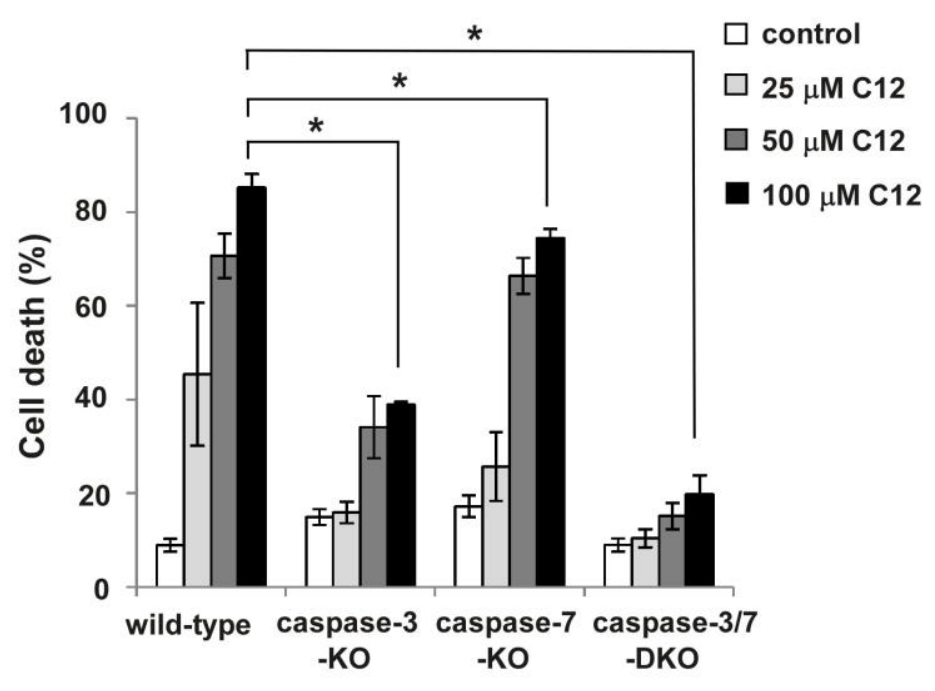

C

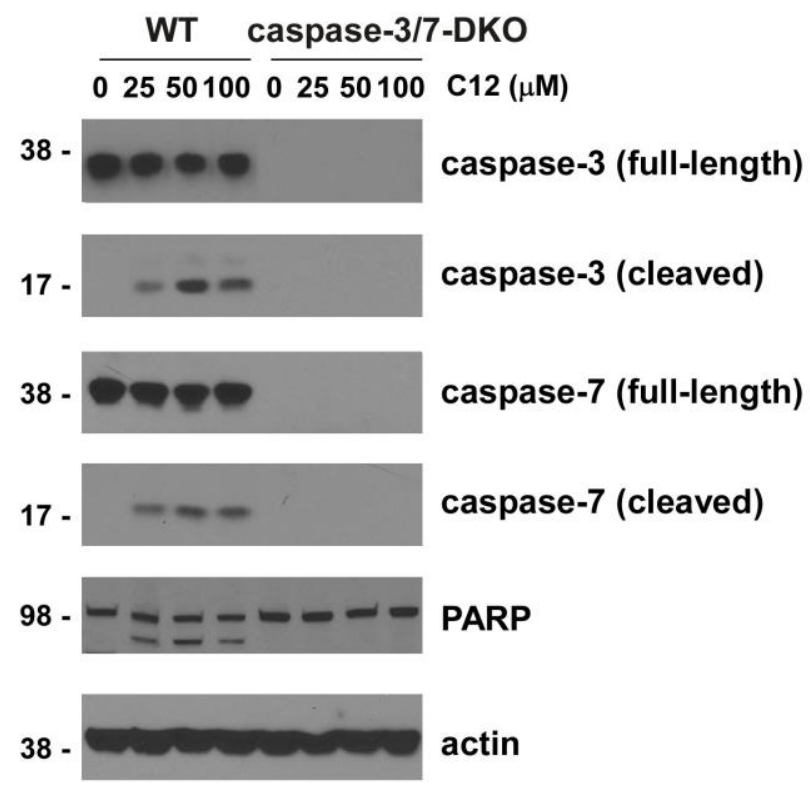


Figure 2.1. Caspase-3 and caspase-7 are required for $\mathrm{C} 12$-induced cell death. (A) The expression of caspase-3 and caspase-7 in the indicated MEFs was examined by western blots. The molecular weight markers were labeled on the left (kD). (B) MEFs were treated with $\mathrm{C} 12$ for 48 hours, and cell viability was measured by propidium iodide exclusion. The data are presented as means \pm standard deviations of three independent experiments. Asterisks indicate $\mathrm{P}<$ $0.01\left(^{*}\right)$; Student's unpaired t test. ns, no significance. (C) Activation of caspase3 and caspase- 7 was determined by western blot. Whole cell extracts of MEFs were acquired following 16 hour-treatment of $\mathrm{C} 12$. The molecular weight markers were labeled on the left $(k D)$. 
2.3.2. $\mathrm{C} 12$-induced MOMP is independent of caspase-3 and caspase-7. Mitochondrial outer membrane permeabilization (MOMP) has been recognized to be a "no-return" step in both intrinsic and extrinsic apoptotic pathways (105, 106). To further explore C12-initiated apoptotic signaling, we studied the involvement of caspase- 3 and caspase- 7 in two key events of MOMP: depolarization of mitochondrial membrane potential ( $\Delta \psi$ mito $)$ and release of cytochrome $\mathrm{c}$ from mitochondria into the cytosol. Depolarization of $\Delta \psi$ mito was evaluated by determining the changes in fluorescence with the voltagedependent dye $\mathrm{JC} 1$ being released from mitochondria into the cytosol and nucleus. Within minutes of $\mathrm{C} 12$ exposure, mitochondrial $\Delta \psi$ mito in WT MEFs became almost completely depolarized to a level close to the complete depolarization induced by the ionophore FCCP (Figure 2.2A), consistent with studies in other cell types $(65,95,98)$. Importantly, C12 triggered similar depolarization of $\Delta \psi$ mito in both WT and caspase-3/7-DKO MEFs (Figure 2.2B), indicating that MOMP induced by $\mathrm{C} 12$ occurs upstream of "effector" caspase activation. 
A
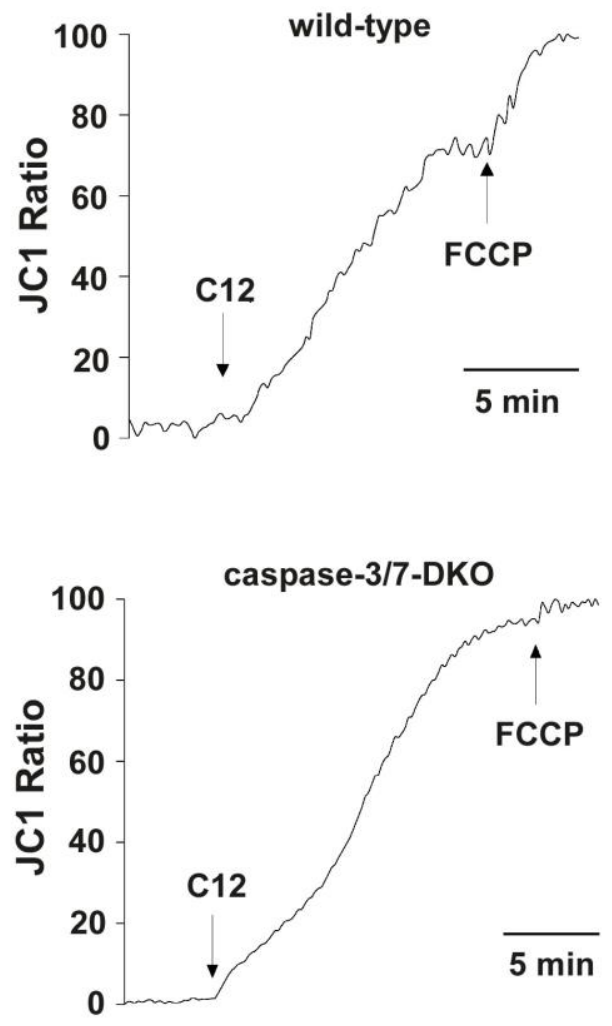

B

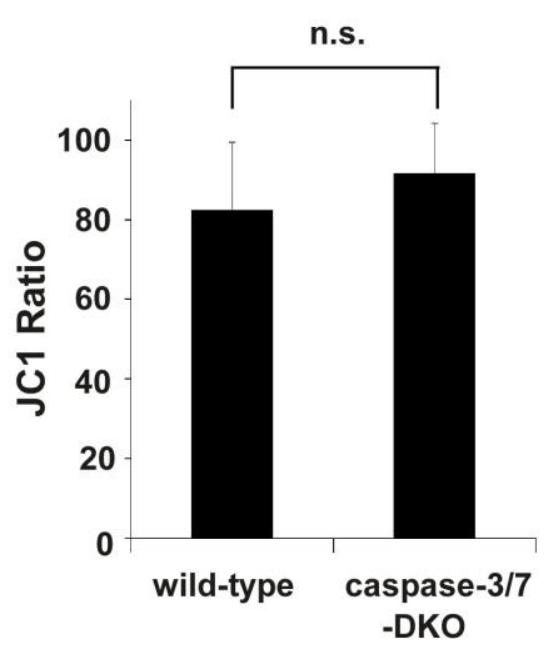


Figure 2.2. Caspase-3 and caspase-7 are not required for mitochondrial depolarization induced by $\mathrm{C} 12$.

(A) MEFs from WT and caspase-3/7-DKO mice were loaded with the mitochondrial potential dye JC-1, and fluorescence was measured using imaging microscopy during sequential treatment with $50 \mu \mathrm{M} \mathrm{C12}$ and $10 \mu \mathrm{M}$ FCCP. Typical results from three independent experiments are shown. (B) Addition of $\mathrm{C} 12$ caused equivalent depolarization of mitochondrial potential in WT and caspase-3/7-DKO MEFs. All data are shown as means \pm standard deviations of three independent experiments. Student's unpaired $t$ test. ns, no significance. 
2.3.3. C12-induced MOMP occurs independent and upstream of caspase-3/7 activation.

Immunofluorescence studies were used to evaluate C12-evoked redistribution of cytochrome $\mathrm{c}$ from mitochondria to the cytosol and nuclei. As shown in representative images of both WT and caspase-3/7-DKO MEFs under control conditions, cytochrome $\mathrm{c}$ and the mitochondrial outer membrane protein Tom20 exhibited overlapping punctate and perinuclear distribution in cells that is typical of mitochondrial morphology (Figure 2.3A). In addition, nuclei displayed characteristic oval shape with smooth boundaries that largely excluded cytochrome $\mathrm{c}$ and Tom20. Upon C12 treatment, distribution of cytochrome c was diffuse in the cytosol and nuclei in both WT and caspase-3/7-DKO MEFs, whereas Tom20 maintained its characteristic mitochondrial distribution, demonstrating that mitochondria in WT and caspase-3/7-DKO MEFs were permeabilized with cytochrome $\mathrm{c}$ released into the cytosol and diffused into the nuclei. The redistribution of cytochrome $\mathrm{c}$ was quantitated by calculating the punctate/diffuse index. These quantitative measurements showed that $\mathrm{C} 12$ caused equivalent cytochrome $\mathrm{c}$ release in WT and caspase-3/7-DKO MEFs (Figure 2.3B), while Tom20 was still localized in mitochondria in the presence of C12 (Figure 2.3C). Overall, these results provide more evidence that $\mathrm{C} 12$ triggers MOMP independent of caspase-3 and caspase-7 activation. 
A

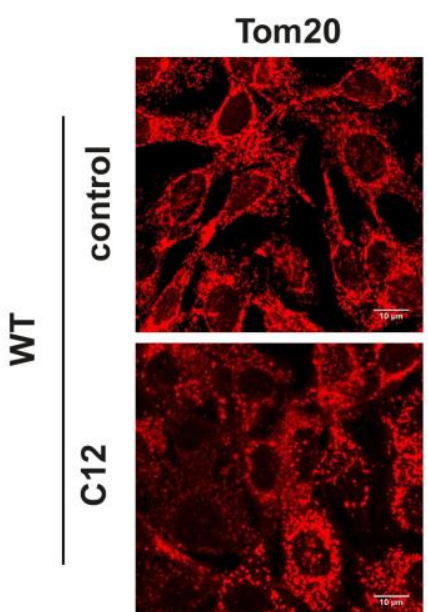

cytochrome c
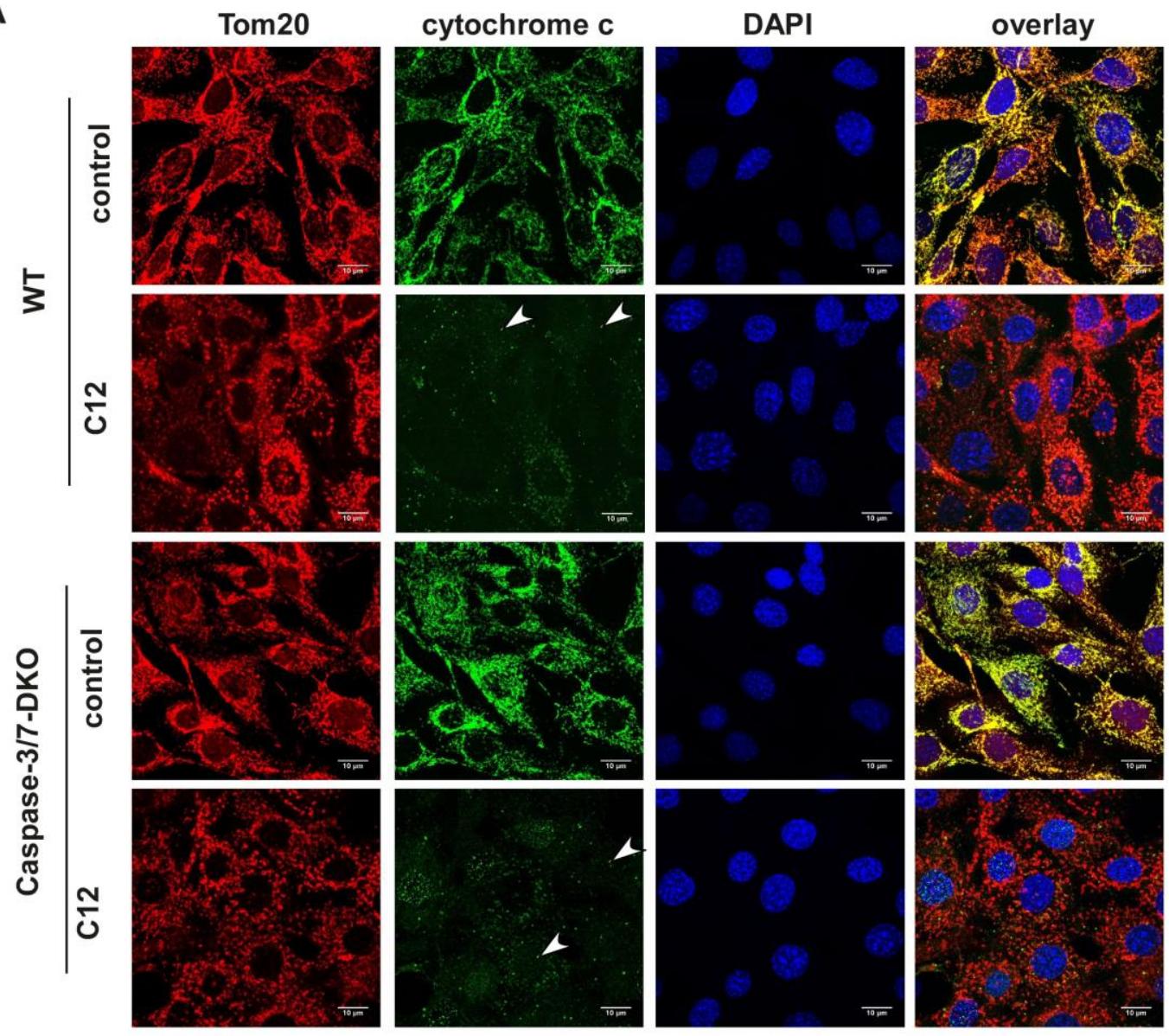

B
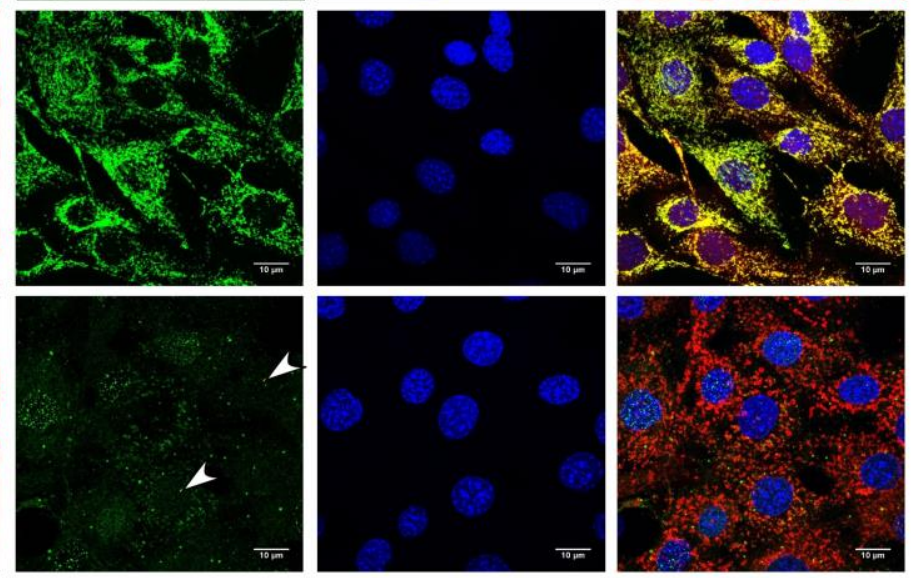

C
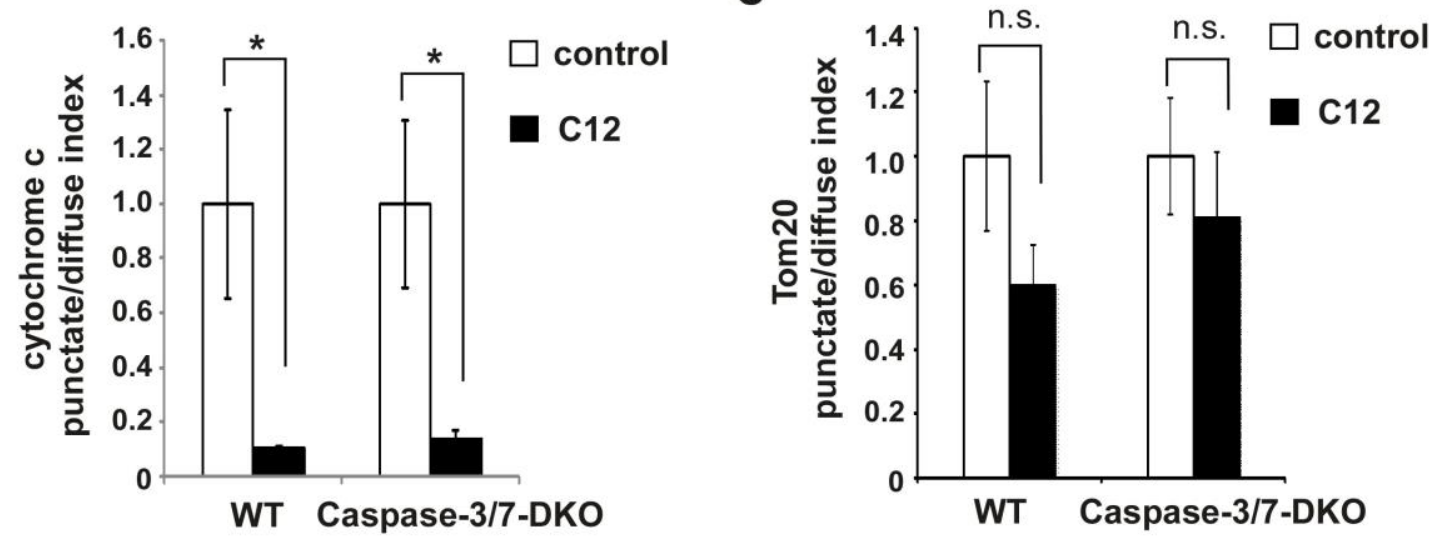
Figure 2.3. C12-induced MOMP occurs upstream of caspase-3/7 activation. (A) Representative confocal images of MEFs treated with either DMSO (control) or $50 \mu \mathrm{M} \mathrm{C} 12$ for 4 hours (typical of three independent experiments. The mitochondrial marker Tom20 is shown in red, cytochrome c in green, and DAPI in blue. Following treatment with $\mathrm{C} 12$, staining of cytochrome $\mathrm{c}$ became diffuse and lost its co-localization with Tom20. Arrowheads indicate cells in which cytochrome $\mathrm{c}$ redistributed from mitochondria to the cytosol and nuclei. (B) Cytochrome $\mathrm{c}$ is released from both WT and caspase-3/7-DKO MEFs to similar degrees upon $\mathrm{C} 12$ exposure. Cytochrome $\mathrm{c}$ release from mitochondrial was quantified by calculating the punctate/diffuse index, defined as the standard deviation of the mean pixel intensity of cytochrome c staining for each cell. Data represent means \pm standard deviations of three independent experiments with at least 100 cells in each experiment. Asterisks indicate $\mathrm{P}<0.05\left({ }^{*}\right)$, Student's unpaired t test. (C) The intracellular distribution of Tom20 was evaluated by determining the punctate/diffuse index. Summary data show the means \pm standard deviations of the punctuate/diffuse index calculated for more than 100 cells in 3 independent experiments. Student's unpaired t test. ns, no significance. 
2.3.4 C12 induces apoptosis independent of caspase-8 in MEFs.

The "initiator" caspase-8 mediates extrinsic pathway initiated at the plasma membrane, whereas the "initiator" caspase-9 is responsible for the mitochondria-dependent intrinsic pathway (92). Activation of extrinsic and intrinsic apoptosis pathways has been reported in cells treated with $\mathrm{C} 12$, but it is not clear to what degree each pathway contributes to cell death $(65,94,97$, 107). To systematically investigate which apoptotic pathway is involved, we first examined MEFs deficient in caspase-8 and their wild-type counterparts (Figure 2.4A). Consistent with a previous report (108), MEFs lacking caspase-8 were resistant to the combined treatment of recombinant human TNF- $\alpha$ protein and the Smac mimetic TL-32711 (Figure 2.4B). Upon exposure with C12, similar levels of cell death (Figure 2.4C) and activated caspase-3/7 (Figure 2.4D) were detected in both WT and caspase-8-KO MEFs. Moreover, C12 triggers activation of caspase- 3 and caspase -7 regardless of caspase- 8 expression (Figure 2.4E). While more caspase-3 was cleaved in WT MEFs exposed to $\mathrm{C} 12$, higher levels of caspase-7 activation were detected in $\mathrm{C} 12$ treated caspase-8-KO MEFs. Similar levels of PARP cleavage was observed in both WT and caspase-8-KO MEFs, which is in agreement with the cell death data (Figure 2.4C). 
A

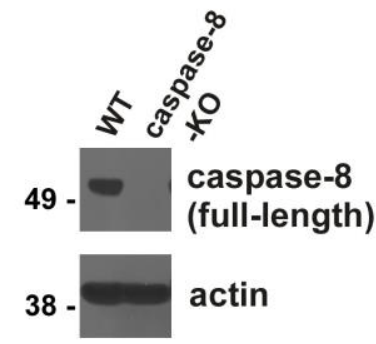

B

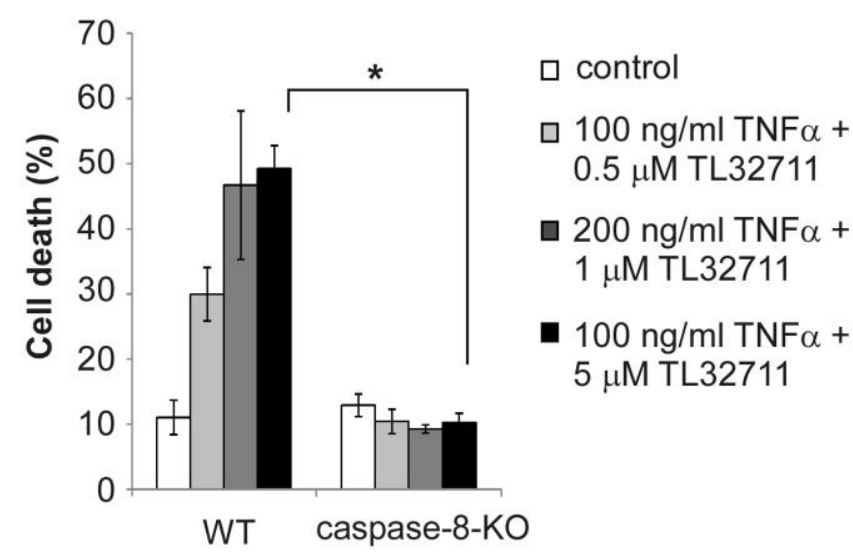

C

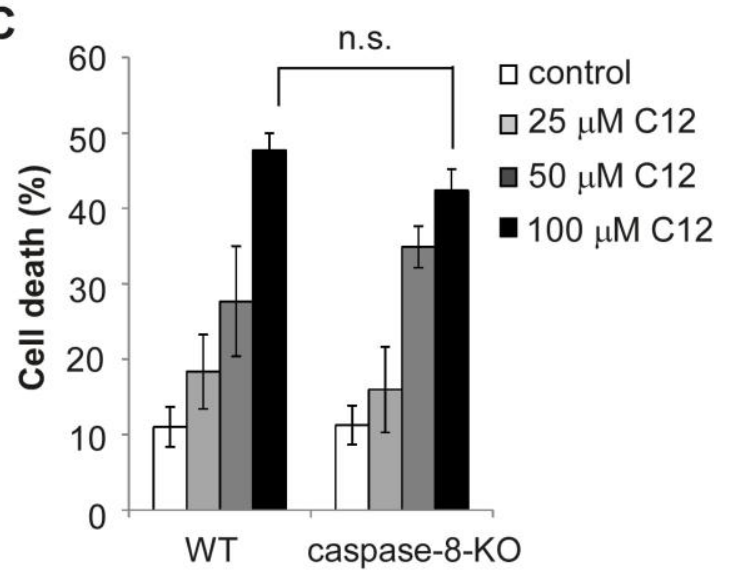

D

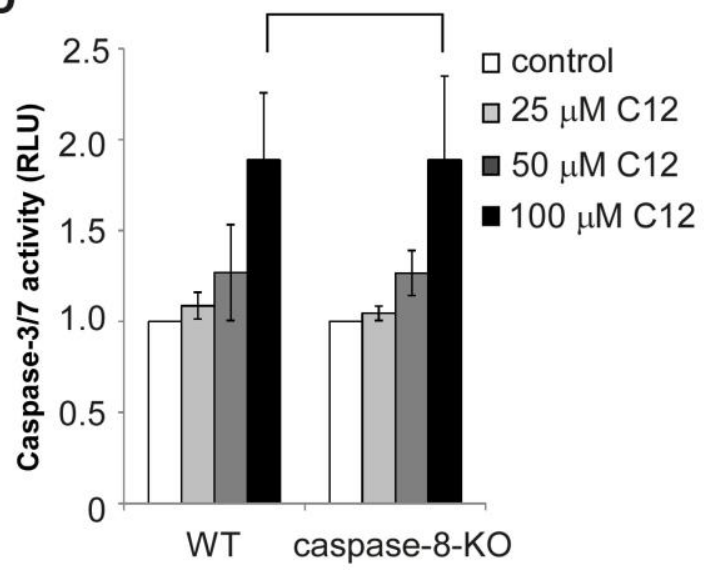

E

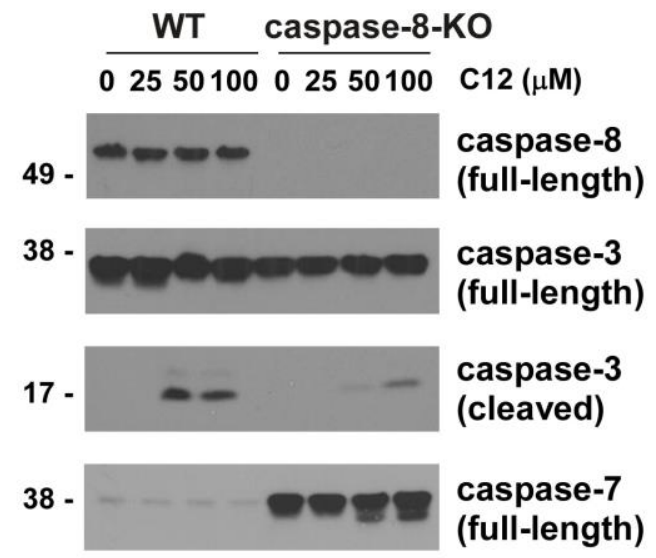

17

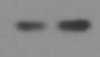

caspase-7 (cleaved)

98

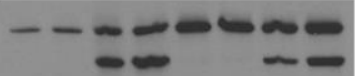

PARP

38 -

actin 
Figure 2.4. Caspase-8 activation is not involved in C12-induced apoptosis.

(A) Caspase-8 expression in MEFs was evaluated by western blot. (B) WT and caspase-8-KO MEFs were cultured with the indicated combination of recombinant human TNF- $\alpha$ and the Smac mimetic TL-32711. Cell viability was determined following 24 hour-exposure by measuring propidium iodide DNA dye exclusion. (C) The cytotoxicity of C12 on WT and caspase-8-KO MEFs was assessed 24 hours after treatment. (D) Caspase-3/7 activities were evaluated 24 hours after $\mathrm{C} 12$ treatment. (E) Upon the treatment of $\mathrm{C} 12$ for 12 hours, activation of caspase-3 and caspase-7 in WT and caspase-8-KO MEFs was evaluated by western blot. The molecular weight markers were labeled on the left $(k D)$. All data are shown as means \pm standard deviations of three independent experiments. Asterisks indicate $P<0.01\left({ }^{*}\right)$; Student's unpaired $t$ test. ns, no significance. 
2.3.5. Caspase-8 is not involved in C12-evoked human tumor cell death. To further validate the role of caspase-8 in C12-indcued cell death, we stably reduced caspase-8 expression in human colorectal carcinoma HCT116 and human pancreatic carcinoma Panc-1 by shRNA (Figures 2.5A and 2.5D). As expect, HCT116 and Panc-1 cells with reduced caspase-8 expression exhibited resistance to the combined treatment of TNF- $\alpha$ and the protein synthesis inhibitor cycloheximide (Figures 2.5B and 2.5E). In contrast, C12 caused similar levels of cell death in both HCT116 and Panc- 1 cells regardless of caspase-8 expression (Figures $2.5 \mathrm{C}$ and $2.5 \mathrm{~F}$ ). These data provide more evidence that C12 induces apoptosis independent of capspase-8. 
A

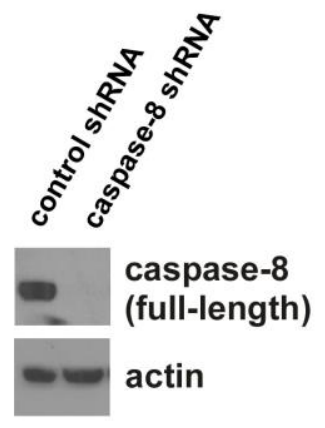

HCT116

C

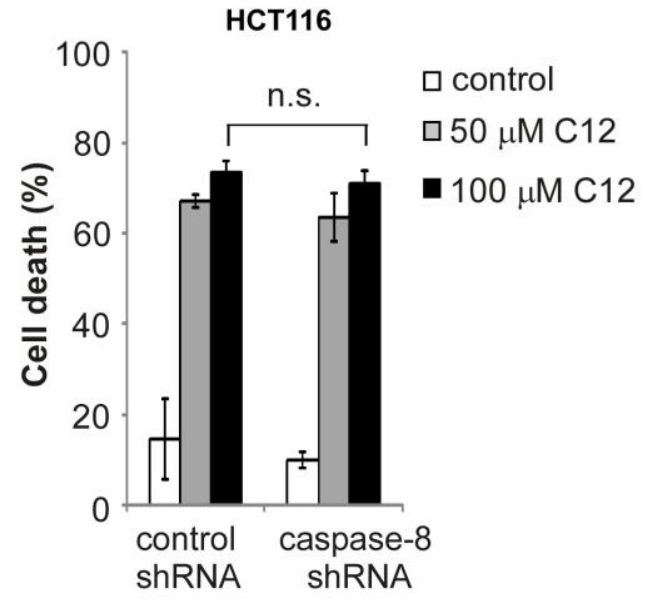

E

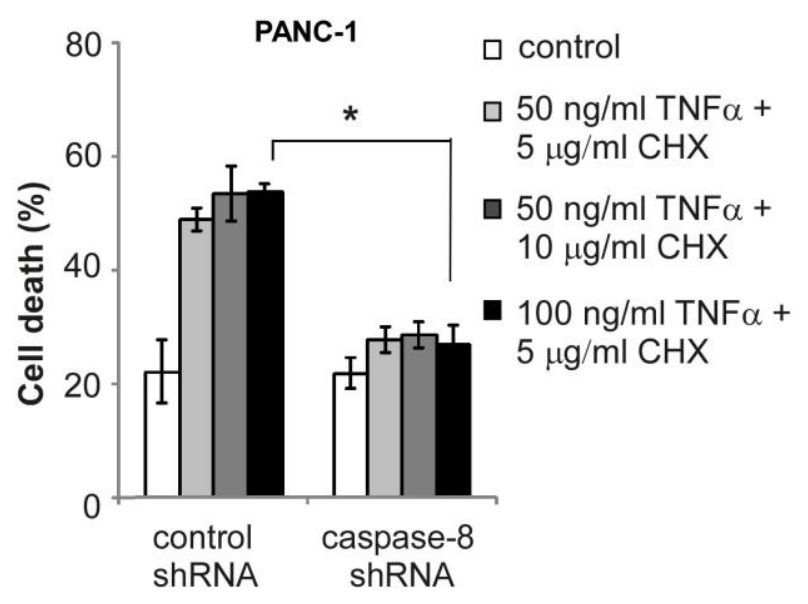

B

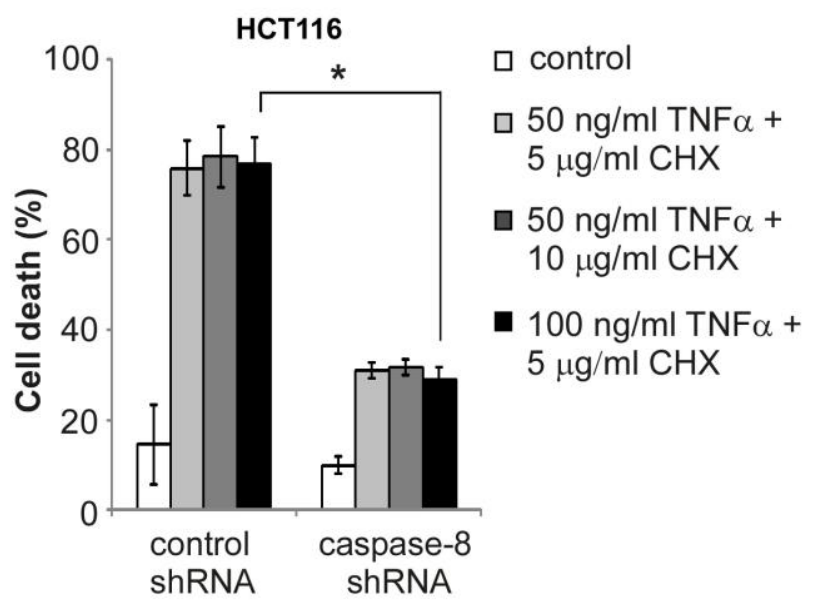

F

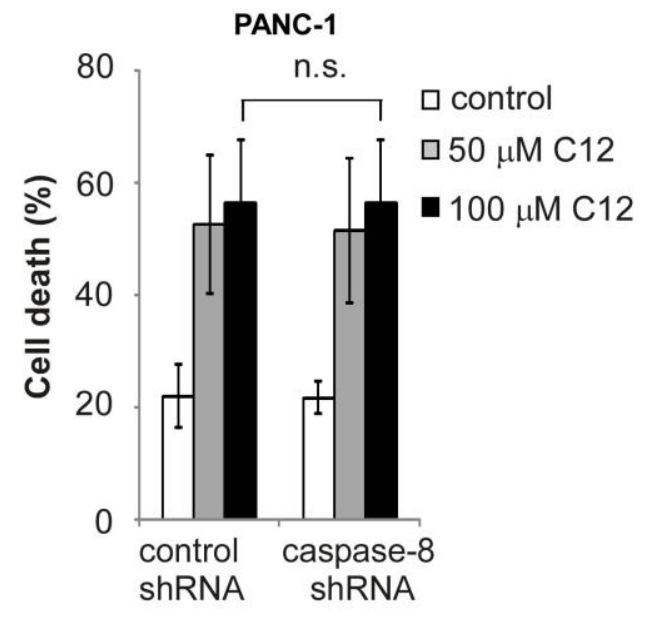


Figure 2.5. Caspase-8 is not involved in C12-induced human tumor cell death. (A) Caspase-8 expression in HCTT116 cells was stably reduced by shRNA. The expression levels of caspase- 8 were determined by western blot. (B) HCT116 cells were cultured with the indicated combination of recombinant human TNF- $\alpha$ protein and cycloheximide (CHX). Cell viability was determined following 48 hour-exposure. (C) The cytotoxicity of $\mathrm{C} 12$ on HCT116 cells was assessed 48 hours after $\mathrm{C} 12$ treatment. (D) Stable reduction of caspase-8 expression in Panc-1 cells was evaluated by western blot. (E) TNF- $\alpha$ protein and cycloheximide $(\mathrm{CHX})$ triggered more cell death in vector control Panc-1 cells than caspase-8 shRNA cells following 24 hour exposure. (F) C12 induced equivalent cell death in vector-control and caspase-8-deficient Panc-1 cells after 24 hour treatment. Data are presented as means \pm standard deviations of three independent experiments. Asterisks indicate $P<0.05\left(^{*}\right)$, Student's unpaired t test. ns, no significance. 
2.3.6. $\mathrm{C} 12$ induces MOMP independent of caspase-8.

To further investigate the role of caspase-8 in C12-initiated apoptotic signaling, we studied the involvement of caspase-8 in depolarization of $\Delta \psi$ mito induced by C12. Consistent with its effects on cell viability and caspase-3/7 activation, deficiency in caspase-8 expression failed to affect the ability of $\mathrm{C} 12$ to induce rapid depolarization of $\Delta \psi$ mito (Figure 2.6). These data indicate that the caspase-8-mediated extrinsic pathway is not involved in C12-induced apoptosis in MEFs. 
A
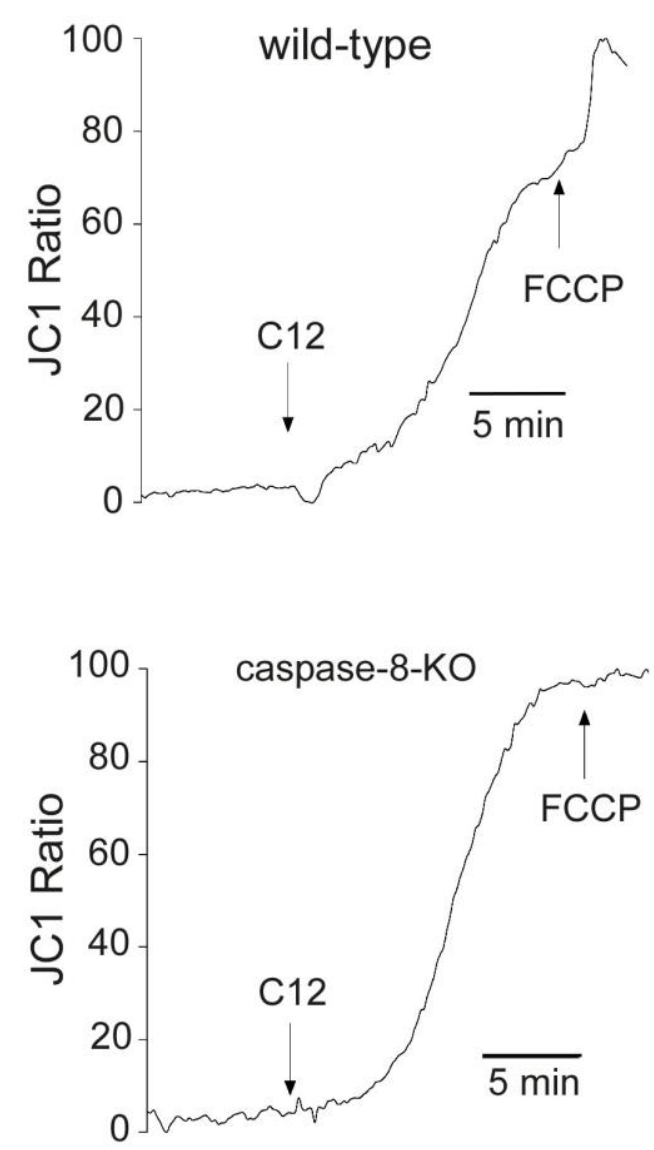

B

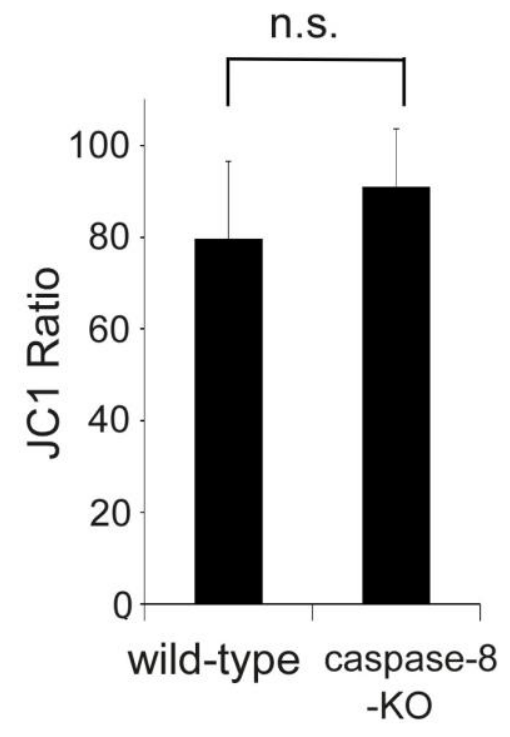


Figure 2.6. C12 induces mitochondrial membrane potential depolarization independent of caspase-8.

(A) Mitochondrial membrane potentials of WT and caspase-8-KO MEFs loaded with JC-1 were determined by fluorescence microscopy upon treatment with 50 $\mu \mathrm{M} \mathrm{C12}$ and $10 \mu \mathrm{M}$ FCCP. Typical results from three independent experiments are shown. (B) C12 caused equivalent mitochondrial depolarization in WT and caspase-8-KO MEFs. Data are presented as means \pm standard deviations of 3 independent experiments. Asterisks indicate $P<0.05\left(^{*}\right)$, Student's unpaired $t$ test. ns, no significance. 
2.3.7. C12 exclusively triggered mitochondria-dependent intrinsic apoptosis pathway.

Next, we investigated whether the "initiator" caspase-9 was responsible for C12-induced apoptosis. For this purpose, MEFs lacking caspase- 9 expression and corresponding wild-type MEFs were examined (Figure 2.7A). Two chemotherapeutic drugs etoposide and actinomycin D known to evoke mitochondria-dependent intrinsic apoptosis pathway only induced cell death in wild-type but not in caspase-9-KO MEFs (Figures 2.7B-C). Unlike their wildtype counterparts, MEFs deficient in caspase-9 were completely resistant to C12 treatment (Figure 2.7D). Importantly, C12 failed to evoke any caspase-3/7 activation in MEFs lacking caspase-9 expression (Figure 2.7E). Furthermore, cleavage of caspase-3 and caspase-7 was only found in wild-type MEFs treated with $\mathrm{C} 12$, but not in caspase-9-KO-MEFs (Figure 2.7F). Overall, these data suggested that $\mathrm{C} 12$ induces apoptotic signaling largely through activating the mitochondria-dependent intrinsic apoptotic pathway in MEFs. 


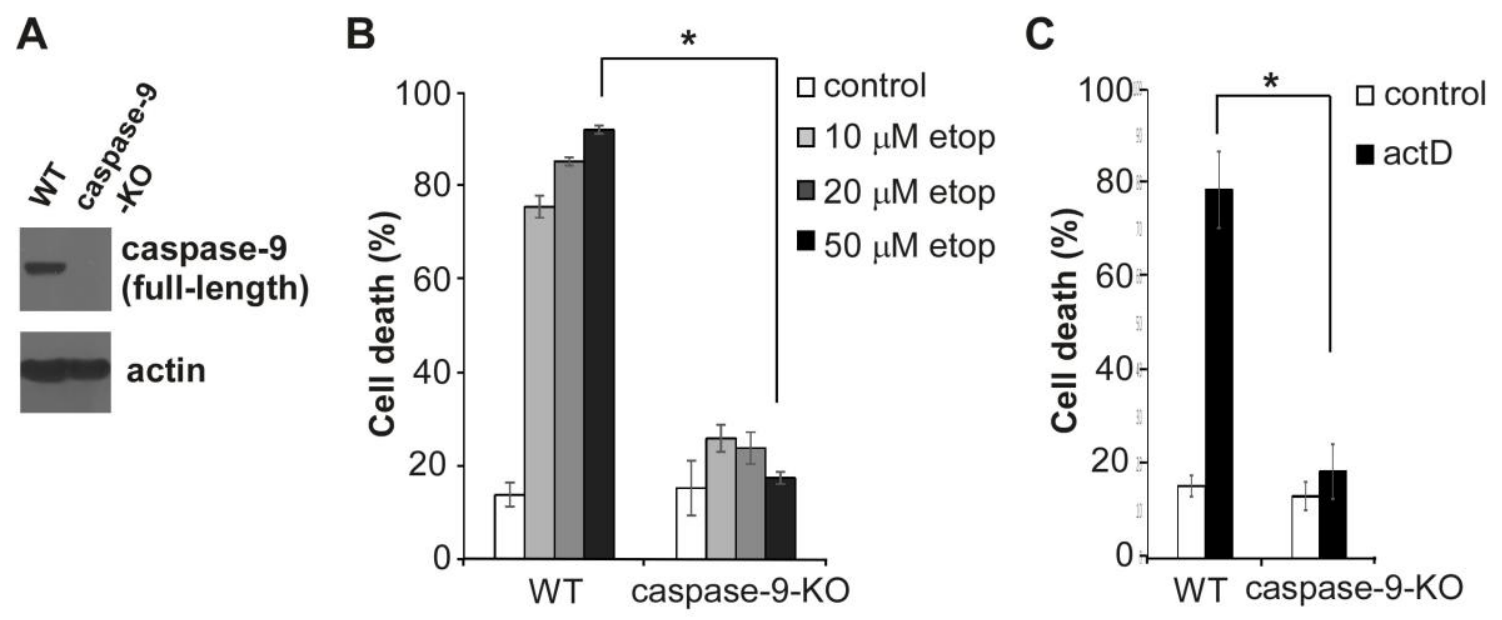

D

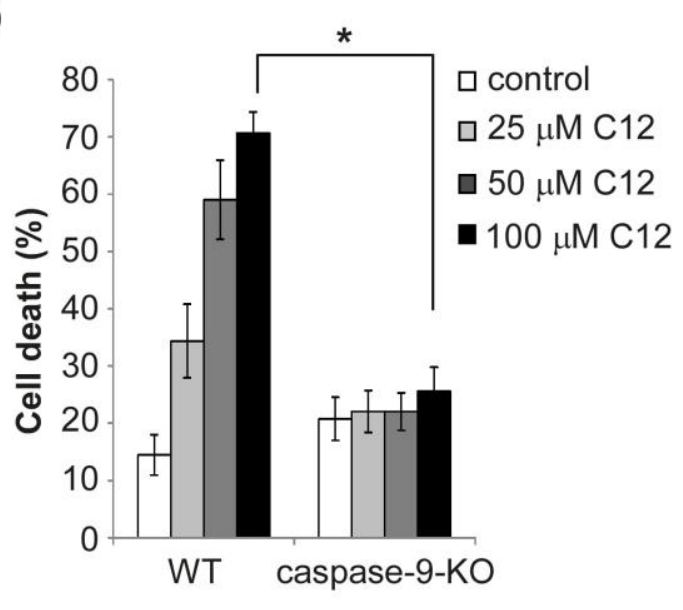

E

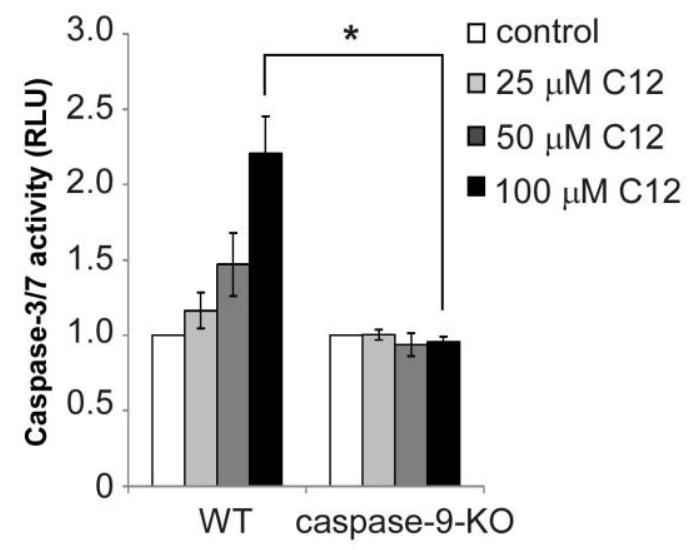

F

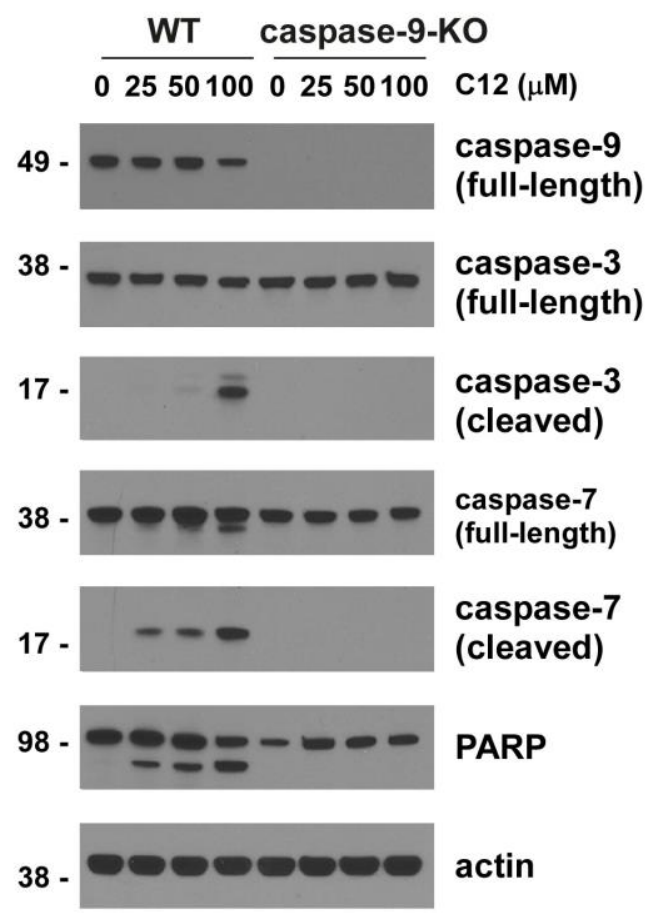


Figure 2.7. C12-induced apoptosis is dependent on caspase-9.

(A) Caspase-9 expression in MEFs was determined by western blot. (B) Wildtype and caspase-9-KO MEFs were treated for 48 hours with various concentrations of etoposide (etop), and cell viability was measured. (C) Wildtype and caspase-9-KO MEFs were cultured with or without $0.5 \mu \mathrm{g} / \mathrm{ml}$ actinomycin D (actD) for 24 hours, and cell viability was evaluated. (D) Cell viabilities of wild-type and caspase-9-KO MEFs were measured 48 hours after treatment with different doses of C12. (E) Caspase-3/7 activities were determined for wild-type and caspase-9-KO MEFs treated with different doses of $\mathrm{C} 12$ for 24 hours. (F) Activation of caspase-3 and caspase- 7 was evaluated by western blot following 16 hour-exposure of $\mathrm{C} 12$. The molecular weight markers were labeled on the left $(\mathrm{kD})$. All the data are shown as means \pm standard deviations of three independent experiments. Asterisks indicate $\mathrm{P}<$ $0.05\left(^{*}\right)$, Student's unpaired t test. ns, no significance. 
2.3.8. $\mathrm{C} 12$ induced MOMP independent of caspase-9.

To further explore the roles of caspase- 9 in MOMP mediated by $\mathrm{C} 12, \Delta \psi$ mito was measured in WT and caspase-9-KO MEFs. In contrast to its effects on cell viability and caspase-3/7 activation (Figures 2.7), deficiency in caspase-9 expression did not affect the ability of $\mathrm{C} 12$ to induce rapid depolarization of $\Delta \psi$ mito (Figure 2.8), indicating that caspase-9 was not involved in C12- induced MOMP. To further validate this notion, we examined cytochrome $\mathrm{c}$ redistribution from mitochondria to the cytosol and nuclei upon $\mathrm{C} 12$ exposure using immunofluorescence staining. While $\mathrm{C} 12$ evoked the release of cytochrome $\mathrm{c}$ from mitochondria to the cytosol and nuclei regardless of caspase-9 expression, Tom20 displayed typical punctate and perinuclear mitochondrial distribution following $\mathrm{C} 12$ treatment (Figure 2.9A). Moreover, C12 caused similar level of cytochrome c release from mitochondria in WT and caspase-9-KO MEFs, whereas Tom20 was retained in mitochondria (Figures 2.9B-C). Taken together, these data indicated that $\mathrm{C} 12$ causes acute MOMP independent of any "initiator" caspase, suggesting that the effects of C12 on MOMP might be attributed to its direct action on mitochondria. 
A

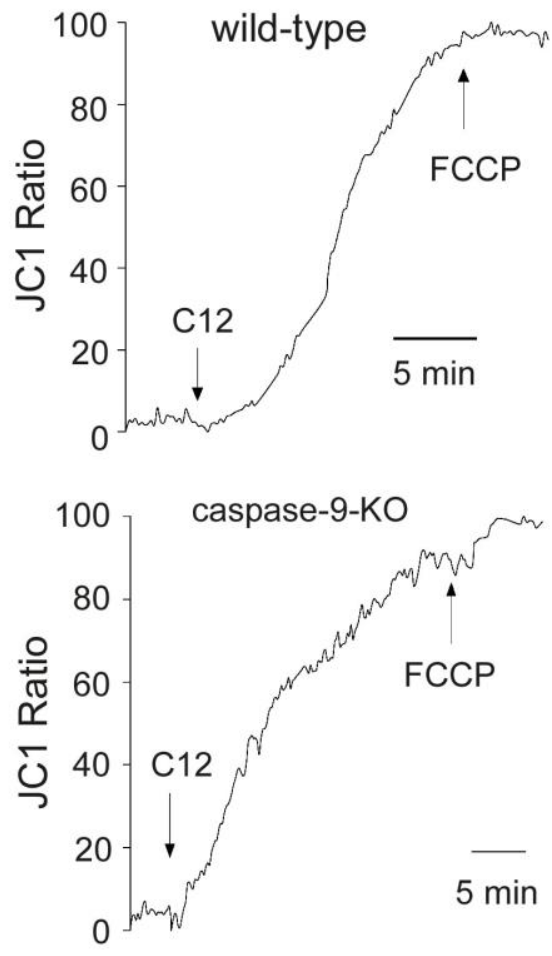

B

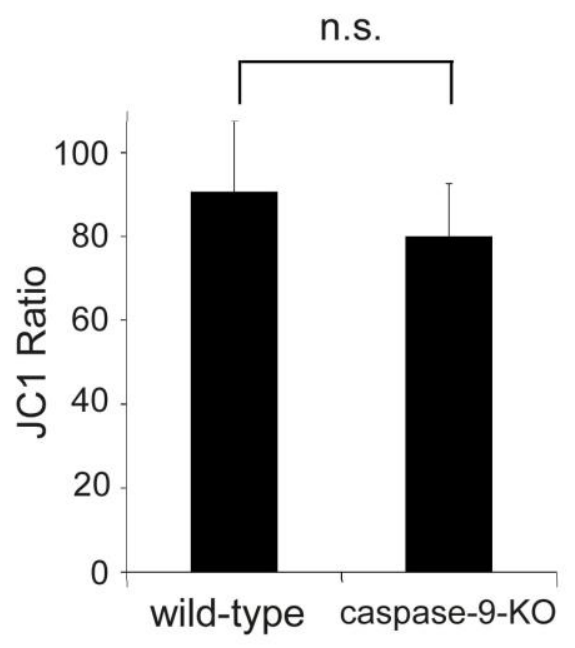


Figure 2.8. C12-induced mitochondrial potential decrease is independent of caspase-9.

(A) WT and caspase-9-KO MEFs were loaded with JC-1, and fluorescence was measured using imaging microscopy during sequential treatment with $50 \mu \mathrm{M}$ $\mathrm{C} 12$ and $10 \mu \mathrm{M}$ FCCP. Typical results from three similar experiments are shown. (B) Summary of depolarization of $\Delta \psi$ mito in WT and caspase-9-KO MEFs. The data are shown as means \pm standard deviations of 3 independent experiments like those in (A). Student's unpaired t test. ns, no significance. 

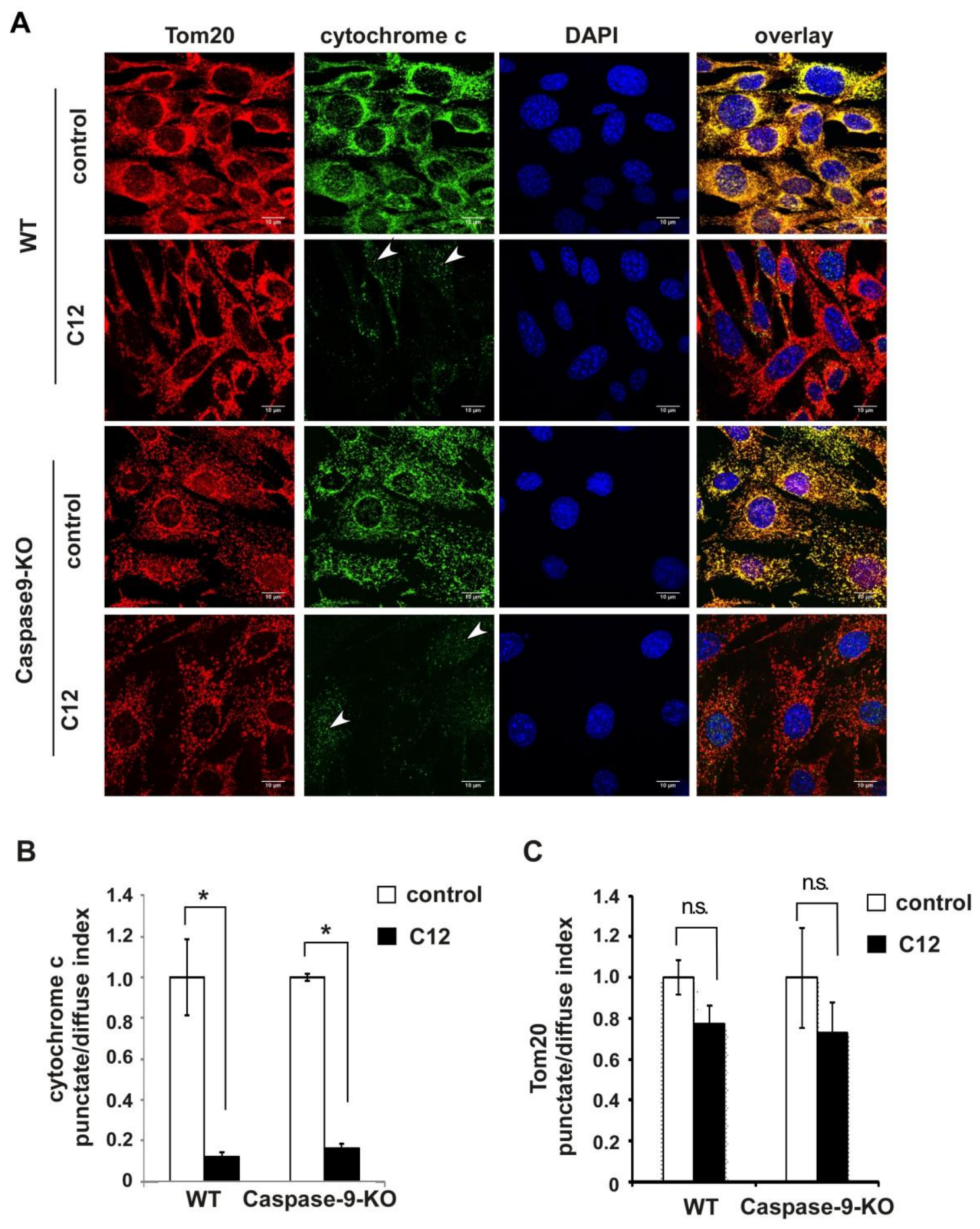
Figure 2.9. C12-induced caspase-9 activation occurs downstream of mitochondrial membrane permeabilization.

(A) Representative confocal images of MEFs treated with either DMSO (control) or $50 \mu \mathrm{M} \mathrm{C12}$ for 4 hours. Tom20 is shown in red, cytochrome $\mathrm{c}$ in green, and DAPI in blue. Arrow heads indicate cells with cytochrome c diffusing in the cytosol and nuclei. (B) Summary data showing cytochrome c release upon $\mathrm{C} 12$ exposure. Cytochrome c release from mitochondria was quantified by calculating the scaled punctate/diffuse index. Data are presented as mean \pm standard deviations of the punctuate/diffuse index calculated for 3 independent experiments with at least 100 cells in each experiment. Asterisks indicate $\mathrm{P}<$ $0.05\left(^{*}\right)$, by Student's unpaired $t$ test. (C) The punctate/diffuse index of intracellular Tom20 was calculated. The data are the means \pm standard deviations of 3 independent experiments with at least 100 cells in each experiment. ns, no significance. Student's unpaired t test. 
2.3.9. $\mathrm{C} 12$ depolarizes mitochondria without influencing mitochondrial morphology.

Changes in mitochondrial morphology during certain paradigms of apoptosis have been associated with depolarization of mitochondria and subsequent release of cytochrome c (109). To investigate the possible involvement of mitochondria morphology in C12-induced mitochondrial depolarization, we carried out experiments to evaluate mitochondrial morphology and membrane potential using time-lapse confocal microscopy on single A549 cells. To this purpose, fluorescent signals of GFP targeted to mitochondrial matrix and the mitochondrial potential indicator TMRE were recorded simultaneously to measure mitochondrial morphology and membrane potential respectively. As shown in Figure 2.10, C12 depolarized mitochondrial potential within 10 minutes of addition to the live cells. Following an established protocol (110), we evaluated mitochondrial morphology by measuring mitochondrial interconnectivity and elongation. C12 did not appear to alter mitochondrial morphology within the time window of the measurement, indicating that $\mathrm{C} 12$ triggered mitochondrial potential depolarization occurs without changes in mitochondrial morphology. 
A
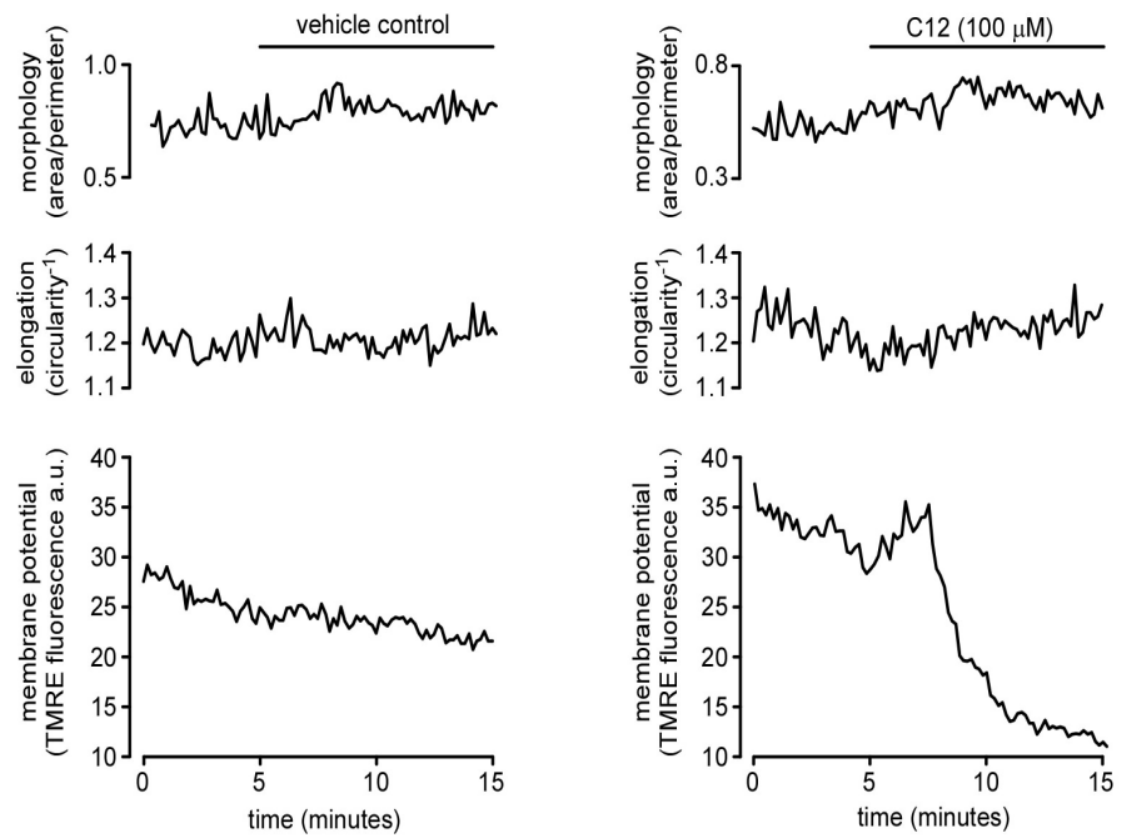

B
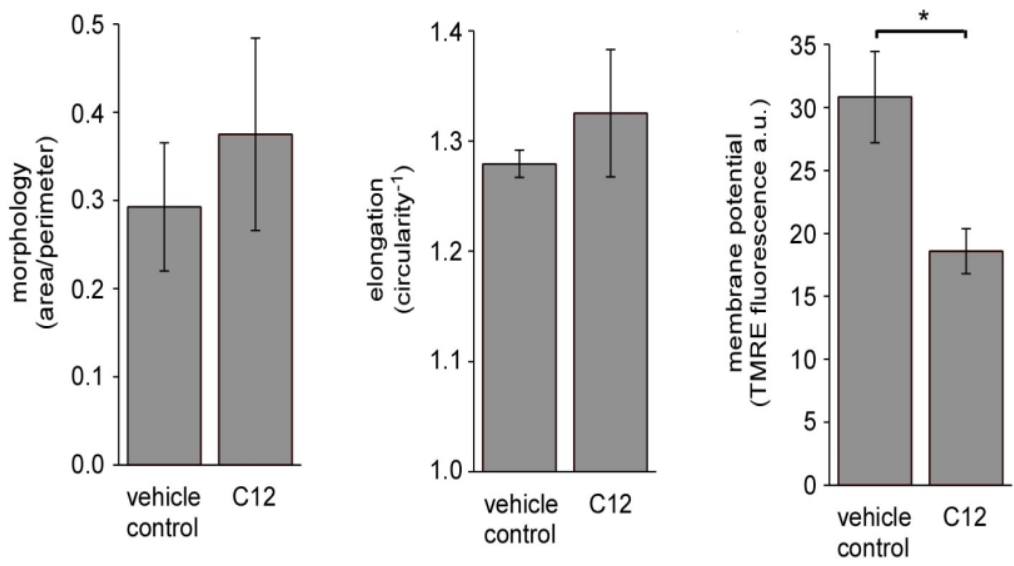
Figure 2.10. C12 depolarizes mitochondrial membrane potential without affecting mitochondrial morphology.

(A) Time-lapse confocal microscopy on single A549 cells was carried out in the presence of the vehicle control DMSO or $100 \mu \mathrm{M} \mathrm{C12}$. Fluorescent signals of mitochondrial matrix-targeted GFP and TMRE were recorded simultaneously to evaluate mitochondrial morphology and membrane potential respectively. Typical traces of mitochondrial area/perimeter ratio, inverse circularity, and membrane potential of a single cell were shown. (B) Summary data (mean \pm SEM) pooled from three independent coverslips run on two separate days depicting morphology and membrane potential at time 0 and after 10 minutes exposure to vehicle control or $\mathrm{C} 12$. Asterisk indicates $\mathrm{P}<0.05\left(^{*}\right)$ by student's paired $t$ test. 
2.3.10. Lysosomes are not involved in cell death induced by $\mathrm{C} 12$.

There is evidence of cross-talk between mitochondria-mediated apoptosis and lysosomal cell death (111). Given the rapid depolarization of $\Delta \psi$ mito induced by C12, it is possible that lysosomes are involved in C12-triggerred caspaseindependent MOMP. The oxidative stress inducer hydrogen peroxide $\left(\mathrm{H}_{2} \mathrm{O}_{2}\right)$ has been shown to cause lysosomal membrane permeabilization (LMP) and subsequent apoptosis, which is blocked by a highly potent iron chelator desferrioxamine (DFO) $(112,113)$. Following pre-incubation with DFO, wildtype and caspase-9-KO MEF cells were treated with $\mathrm{H}_{2} \mathrm{O}_{2}$ and $\mathrm{C} 12$ respectively. As expected, both $\mathrm{H}_{2} \mathrm{O}_{2}$ and $\mathrm{C} 12$ failed to induce cell death in capsapse-9-KO cells. Consistent with previous studies, the cell death induced by $\mathrm{H}_{2} \mathrm{O}_{2}$ was markedly reduced by DFO pre-treatment (Figure $2.11 \mathrm{~A}$ ). In contrast, DFO did not have any significant influence on cell death evoked by C12 (Figure 2.11B), providing evidence that lysosomal cell death is unlikely involved in C12-induced cell death. 
A

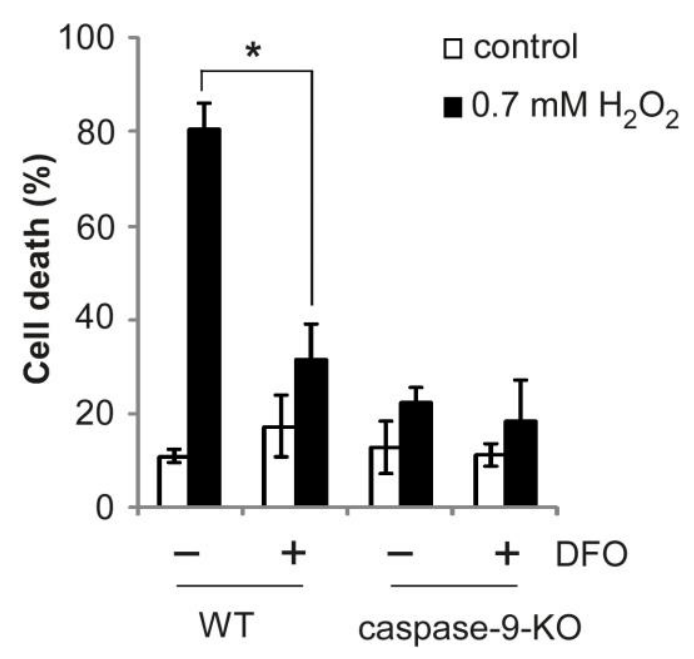

B

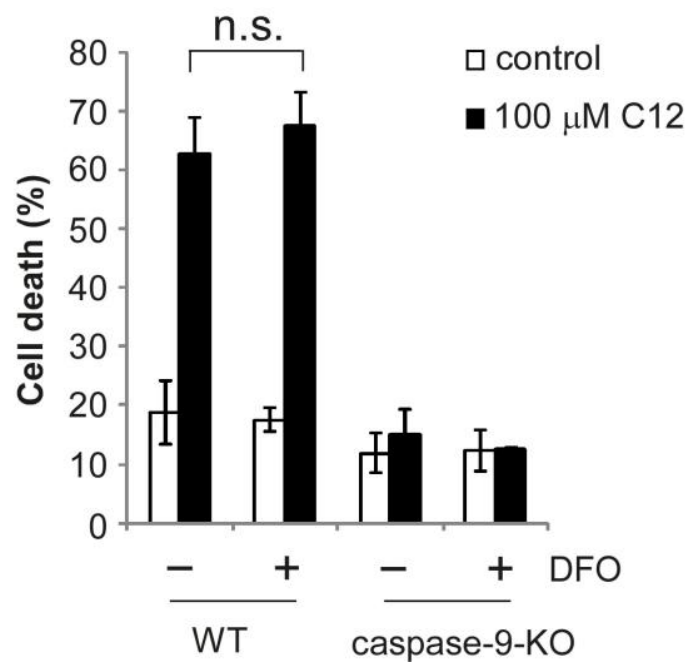

Figure 2.11. Lysosomal membrane permeabilization is not involved in cell death induced by $\mathrm{C} 12$.

Wild-type and caspase-9-KO MEFs were cultured with or without $1 \mathrm{mM}$ DFO for 2 hours. Following DFO removal, $0.7 \mathrm{mM} \mathrm{H}_{2} \mathrm{O}_{2}$ or $100 \mu \mathrm{M} \mathrm{C} 12$ was added to cells respectively. Cell death was measured 24 hours after $\mathrm{H}_{2} \mathrm{O}_{2}$ treatment (A) or 48 hours after $\mathrm{C} 12$ treatment (B). Data are shown as means \pm standard deviations of three independent experiments. Asterisks indicate $\mathrm{P}<0.05\left({ }^{*}\right)$, Student's unpaired t test. ns, no significance 


\subsubsection{C12 directly induces MOMP in vitro.}

Since $\mathrm{C} 12$ depolarized $\Delta \psi$ mito starting within 1 minute and achieving complete effect within 10-15 minutes independent of both "initiator" caspases and "effector" caspases (Figures 2.2, 2.6 and 2.8), we reasoned that C12 could possess activities that directly permeabilize mitochondria. To this purpose, we examined the effects of $\mathrm{C} 12$ on mitochondrial outer membrane integrity in vitro. Mitochondria isolated from WT MEFs were exposed to DMSO (control) or to 30 or $300 \mu \mathrm{M} \mathrm{C12.} \mathrm{We} \mathrm{assessed} \mathrm{the} \mathrm{amount} \mathrm{of} \mathrm{cytochrome} \mathrm{c}$ released from the mitochondria using western blot analysis (Figure 2.12A). In a manner dependent on [C12], less cytochrome $\mathrm{c}$ was detected in mitochondrial fractions, with concomitant increase of cytochrome $\mathrm{c}$ in released fractions (Figure 2.12B). These data indicated that $\mathrm{C} 12$ is able to permeabilize mitochondria directly in vitro. Overall, our data suggest that C12 evokes a mitochondrial apoptotic signaling pathway in which $\mathrm{C} 12$ directly permeabilizes mitochondria, leading to activation of apoptosis signaling (Figure 2.12C). 
A
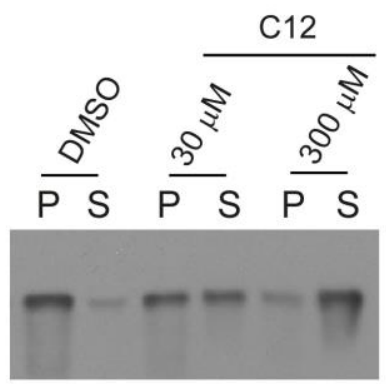

cytochrome c

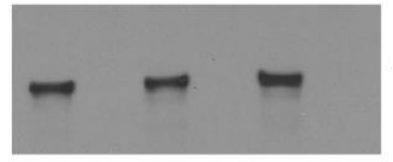

Tom40

p: pellet (mitochondrial fraction)

$\mathrm{s}$ : supernatant (released fraction)
B

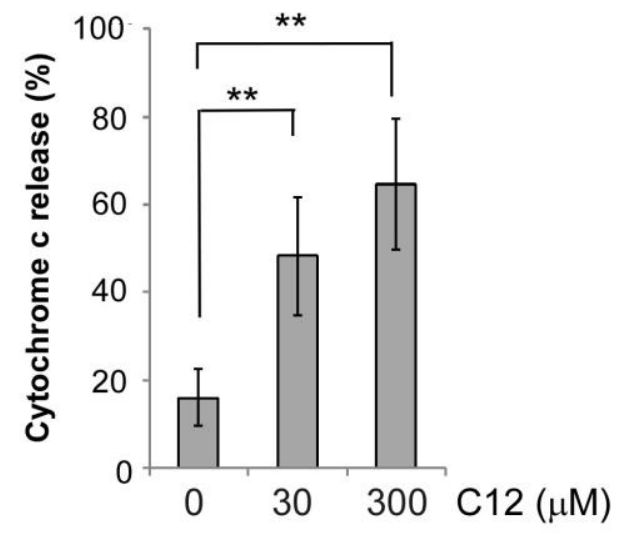

C

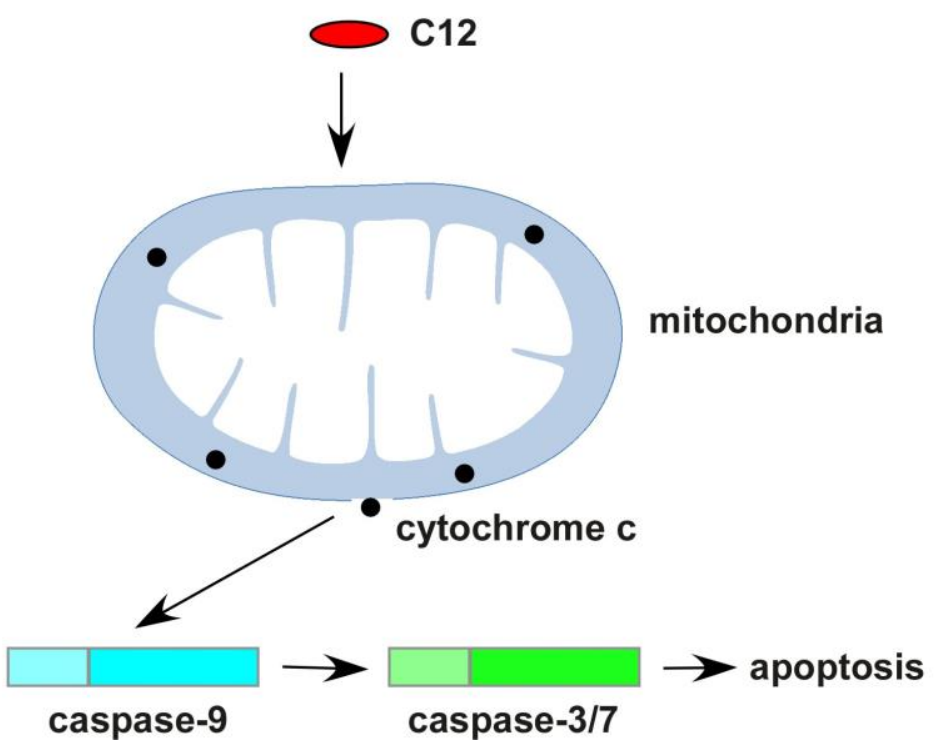


Figure 2.12. C12 directly induces MOMP in vitro.

(A) Mitochondria were isolated from wild-type MEFs and incubated with $\mathrm{C} 12$ for 1 hour. Release of cytochrome c from mitochondria was determined by western blot. (B) The intensities of cytochrome $\mathrm{c}$ in mitochondrial $(\mathrm{P})$ and cytosolic (S) fractions shown in (A) were quantified using Image $(\mathrm{NIH})$. Cytochrome $\mathrm{c}$ release is represented as a percentage of the sum of the protein intensity in mitochondrial and released fractions. Mean \pm standard deviation for four independent experiments are shown. Asterisks indicate $\left.P<0.01{ }^{* *}\right)$ by Student's unpaired t test. (C) C12 permeabilizes mitochondria, subsequently activating apoptotic-signaling pathway. 


\subsection{DISCUSSION}

The quorum-sensing molecule $\mathrm{C} 12$ evokes apoptosis in a variety of mammalian cells $(65,66,69,87,88)$. Several signaling pathways leading to apoptosis have been associated with $\mathrm{C} 12$ cytotoxicity. In addition to activating the intrinsic apoptosis pathway, C12 also activates caspase-8, implicating a role of the extrinsic apoptosis cascade in C12-induced apoptosis $(65,93,94,97)$. One possible scenario is that $\mathrm{C} 12$ functions as a ligand that is recognized by receptors on the plasma membrane. However, the interplay between the intrinsic and extrinsic pathways and the functions of key molecules involved had not been clearly elucidated previously. In this study, we present evidence that caspase-3/7 and caspase- 9 but not caspase- 8 are essential for $\mathrm{C} 12$-induced apoptotic cell death, indicating that $\mathrm{C} 12$ selectively triggers the mitochondriadependent intrinsic apoptotic pathway. Therefore, the events associated with activating a plasma membrane receptor are likely secondary responses to MOMP.

In mitochondria-mediated intrinsic apoptosis signaling cascade, it is generally believed that MOMP and cytochrome c release occur upstream of caspase- 9 activation, which subsequently leads to activation of caspases-3/7(34).

However, the involvement of caspases-3/7 activation in MOMP has only been carefully examined for limited number of apoptotic stimuli. In the study where caspase-3/7-DKO MEFs was first reported, UV irradiation is found to trigger intrinsic apoptosis pathway through activating caspase-3/7 (104). Unlike their 
wild-type counterparts, cells deficient in caspase-3/7 expression maintain their $\Delta \psi$ mito upon UV irradiation. This result provides evidence that caspase-3/7 could regulate the initial phase of intrinsic apoptosis signaling in certain apoptotic paradigms, raising the possibility of existing a feedforward loop among MOMP, caspase- 9 activation and caspase3/7 activation. In contrast, our studies provide unequivocal evidence that C12 evokes MOMP independent of both "initiator" and "effector" caspases. The dispensable roles of caspases in C12-induced MOMP suggest that C12 may exert its pro-apoptotic effect without the involvement of signaling upstream of mitochondria.

Several signaling pathways linked to apoptosis initiation, including JAK/STAT (66), MAPK and elF2 $\alpha$ pathways (91) have been implicated in C12-evoked cell death. Most of these apoptosis-associated signaling pathways are normally involved in multiple steps of signal transduction, and the biological events reflective of these signaling cascades are largely observed hours following $\mathrm{C} 12$ incubation. In contrast, depolarization of $\Delta \psi$ mito, the earliest step of MOMP (5, 101), is always detected within minutes following C12 exposure and reaches its maximal levels in 20 minutes (Figures 2.2, 2.6 and 2.8; also see (65, 95, 107, 114). Thus, it appears that activation of JAK/STAT, elF2a and MAPK signaling pathways may be secondary to MOMP initiation in the C12-triggered apoptosis cascade. 
C12 has also been demonstrated to trigger the ER stress pathway $(65,90,114$, 115). Release of $\mathrm{ER} \mathrm{Ca}^{2+}$ into the cytosol is observed within minutes of $\mathrm{C} 12$ exposure, which is implicated in C12-induced apoptosis $(65,114,115)$. Since the effects of ER stress on $\Delta \psi$ mito occurs several hours following $\mathrm{ER} \mathrm{Ca}^{2+}$ pool depletion (102), the likelihood that $\mathrm{C} 12$-induced $\mathrm{ER} \mathrm{Ca}^{2+}$ release directly causes depolarization of $\Delta \psi$ mito is low. Furthermore, recent research indicates that ER stress-mediated apoptosis requires the pro-apoptotic Bcl-2 proteins Bak and Bax to permeabilize mitochondria $(102,116,117)$, which is inconsistent with the evidence that $\mathrm{C} 12$ triggers depolarization of $\Delta \psi$ mito independent of Bak and Bax in various cellular systems $(93,95)$. Thus, it is unlikely that ER stress signaling is directly involved in C12-mediated depolarization of $\Delta \psi$ mito. Conceivably, C12 or its metabolite(s) acts directly on mitochondria and permeabilizes them. In support of this notion, our in vitro studies demonstrate that C12 directly permeabilize mitochondria (Figure 2.12).

As a lactone, $\mathrm{C} 12$ is known to be hydrolyzed into a carboxylic acid by paraoxonase 2 (PON2), an enzyme with lactonase and arylesterase activities in vitro (76). Intracellular PON2 has been demonstrated to hydrolyze $\mathrm{C} 12$ and cause subsequent intracellular acidification, which is thought to mediate biological responses to $\mathrm{C} 12$, such as triggering stress signaling (115). However, the association between C12-evoked intracellular acidification and depolarization of $\Delta \psi$ mito remains unclear. Our previous studies demonstrated that PON2 promotes C12-induced apoptosis in various cellular systems (95, 
114). In addition to its localization on the ER membrane and plasma membrane (118), PON2 has been shown also to be localized in mitochondria (73). One possibility is that PON2 cleaves $\mathrm{C} 12$ into a pro-apoptotic metabolite(s) that permeabilizes mitochondria (91). C12 or a PON2-mediated derivative might also react with other molecules (e.g. phosphorylation) in cells to generate secondary metabolite(s) with stronger pro-apoptotic activities. Alternatively, PON2 has anti-oxidant properties that modulate peroxidation of membrane lipids (118) and may regulate intracellular lipid biogenesis $(119,120)$, which might affect mitochondrial outer membrane lipid composition in such a way that C12 preferentially inserts into mitochondria.

Whatever the molecular details of $\mathrm{C} 12$ action on mitochondria, our study has shown that $\mathrm{C} 12$ induces a unique apoptotic signaling pathway in which $\mathrm{C} 12$ or C12 metabolite(s) acts as a mitolytic molecule that acts to directly permeabilize mitochondria, releasing cytochrome $c$ to activate caspases 9,3 and 7 and subsequent downstream signaling leading to apoptosis and cell death 
CHAPTER III

N-(3-OXO-ACYL) HOMOSERINE LACTONE INHIBITS TUMOR GROWTH INDEPENDENT OF BCL-2 PROTEINS

\subsection{INTRODUCTION}

Quorum sensing is a bacterial intercellular communication system that constitutively produces, releases, and detects small diffusible autoinducers that are similar to mammalian hormones (58). The gram-negative human pathogen Pseudomonas aeruginosa uses N-(3-oxododecanoyl)-L-homoserine lactone (C12) as a quorum-sensing signal $(82,83)$.

Recent studies have shown that $\mathrm{C} 12$ is not only important in regulating bacterial virulence genes but also interacts with eukaryotic cells and modulates cell physiology, such as triggering cell death (60). C12 has been demonstrated to cause apoptosis in a variety of cancer cells $(66,69-71)$. C12 induces apoptosis through inhibiting the phosphatidylinositide 3-kinases and Akt/PKB pathway and diminishing STAT3 activities in breast carcinoma cells (66). In pancreatic carcinoma cells, $\mathrm{C} 12$ also triggers apoptotic signaling and inhibits cell migration (70). C12 decreases the expression of thymidylate synthase and enhances the activity of chemotherapeutic agents, 5-fluorouracil (5- FU), Tomudex and Taxol in 
colorectal and prostate cancer cells. Recently, a derivative of C12, 3-oxo-12phenyldodecanoyl-L-homoserine lactone, has been identified as another cancer cell growth inhibitor (69). Comparative SAR analysis demonstrates that long acyl side chains with a 3-oxo substitution are essential for C12's anti-cancer effect (69). In light of its function of triggering tumor cell death, C12 displays promise as a cancer treatment. However, detailed apoptotic signaling of $\mathrm{C} 12$ remain unclear. Additionally, whether or not $\mathrm{C} 12$ cytotoxicity in vitro is relevant; to tumor growth in vivo, has never been studied.

Resistance toward apoptosis is a hallmark of most, perhaps all, types of human cancer $(121,122)$. Bcl-2 proteins are the major regulators of apoptotic signaling pathways and can be classified into anti-apoptotic and pro-apoptotic groups. Anti-apoptotic $\mathrm{Bcl}-2$ proteins; such as $\mathrm{Bcl}-2$, are considered to protect against mitochondrial outer membrane permeabilization (MOMP) during apoptosis, whereas pro-apoptotic Bcl-2 members such as Bax and Bak promote MOMP (42, 123). The expression of individual $\mathrm{Bcl}-2$ proteins in different types of cancer has been used as an independent prognostic marker (122). Studies in various human tumors showed that loss of Bax expression, or increased expression of Bcl-2, are associated with their resistance to chemotherapy (124-127). Accordingly, one strategy for cancer therapy is to identify agonists that activate apoptotic pathway independent of Bcl-2 proteins in tumor cells (128-130). 
To gain insights into the mechanism of C12-evoked tumor cell apoptosis, we evaluated the cytotoxic effects of $\mathrm{C} 12$ on tumor cells in vitro, the inhibitory effects of $\mathrm{C} 12$ on tumor growth in vivo and the role of pro- and anti-apoptotic $\mathrm{Bcl}-2$ proteins in C12-induced apoptosis were systematically examined. C12 inhibited the tumor growth through inducing apoptosis in a $\mathrm{Bcl}-2$ protein-independent manner. Our study reveals a unique anti-tumor function of $\mathrm{C} 12$, which may lead to the development of new therapeutic agents for cancer. 


\subsection{MATERIALS AND METHODS}

\subsubsection{Reagents}

$\mathrm{N}$-(3-oxododecanoyl)-homoserine lactone (C12), polybrene and actinomycin D were purchased from Sigma (St. Louis, MO). Propidium iodide (PI), CMH2DCFDA (5-(and-6)-chloromethyl-2', 7'-dichlorodihydrofluorescein diacetate, acetyl ester), TMRE (tetramethylrhodamine, ethyl ester) and TOTO-3 were obtained from Invitrogen (Carlsbad, CA). Unless otherwise stated, all reagents were dissolved in dimethyl sulfoxide (DMSO). Dulbecco's Modified Eagle's Medium (DMEM), penicillin/streptomycin, trypsin, and L-glutamine were obtained from Mediatech (Manassas, VA), and fetal bovine serum was purchased from Gemini (Broderick, CA). Caspase-Glo assay 3/7 kit was purchased from Promega (Madison, WI). Antibodies (Abs) for western blot were anti- $\beta$-actin mAb (Sigma), anti-Bcl-2 mAb; anti-Bax pAb (Santa Cruz; Dallas, TX), anti-Bak pAb (Millipore; Billerica, MA), anti-Mcl-1 pAb (Abcam; Cambridge, MA), anti-Noxa pAb (Novus; Littleton, CO), anti-Puma pAb (ProSic; Poway, CA) peroxidaseconjugated goat anti-rabbit IgG (Thermo Fisher; Waltham, MA) and peroxidaseconjugated goat anti-mouse IgG (Thermo Fisher).

\subsubsection{Plasmids}

pBABE-hBcl-2-IRES-EGFP was generated by cloning human Bcl-2 cDNA into pBABE-IRES-EGFP. pMIG-hMcl-1-IRES-EGFP and pMIG-hBcl-xL-IRES-EGFP was a gift from Dr. Levi Beverly (University of Louisville). The plasmid identities were validated by sequencing. Lentiviral human PON2 shRNA plasmid was purchased from Santa Cruz. 


\subsubsection{Retrovirus production and cell culture}

Retrovirus were generated as described previously (99). HCT116 cells expressing different levels of Bak and Bax were obtained from Dr. Richard Youle (National Institutes of Health). HCT116 cells stably expressing Noxa shRNA or the control luciferase shRNA were described previously (131). Puma-deficient HCT116 cells were acquired from Dr. Bert Vogelstein (John Hopkins University). NSCLC cell line A549 cells were obtained from ATCC, which were produced from adenocarcinomic alveolar basal epithelial cells of a lung cancer patient (132). NCl-H1299 cells were also acquired from ATCC. A549 cells expressing Mcl-1shRNA or control scramble shRNA were described previously (133). A549 cells overexpressing $\mathrm{Bcl}-2, \mathrm{Bcl}-\mathrm{XL}$ or $\mathrm{Mcl}-1$ were produced by retroviral infection as described previously (99). NHBE cells were purchased from Lonza (Walkersville, MD). hT/LT/Ras HBE cells were obtained from Professor Barrett Rollins (Harvard Medical School). Lewis Lung Carcinoma cells, NCl-H1299 and A549 cells were cultured as described previously (134). NHBE and hT/LT/Ras cells were grown in BEGM supplemented with SingleQuots (LONZA). HCT116 cells were grown as described previously (135). Cells were all cultured in a 5\% $\mathrm{CO}_{2}$ humidified incubator at $37^{\circ} \mathrm{C}$.

\subsubsection{Cell death analysis and Chou-Talalay synergism assay}

Cell viability was determined by propidium iodide exclusion using flow cytometry as described previously (134). The synergistic effect of C12 and ABT-737 on cell viability was evaluated by Chou-Talalay median dose effect assay. IC 50 of $\mathrm{C}_{12}$ 
and ABT-737 on $\mathrm{NCl}-\mathrm{H} 1299$ or $\mathrm{A} 549$ was first determined. Various combinations of $\mathrm{C} 12$ and $\mathrm{ABT}-737$ at a constant ratio above or below their $\mathrm{IC}_{50}$ values were added to cells. Following incubation for the indicated time, cell viability was evaluated and Fa (fractional activity) was determined as the ratio between the cell death levels of drug-treated cells and those of untreated control cells. Combination index $(\mathrm{Cl})$ was calculated by the CompuSyn software (Biosoft, Cambridge, UK).

\subsubsection{Caspase-3/7 activity and TUNEL labeling}

Caspase-3/7 activities were measured using a Caspase-Glo assay kit (Promega, Madison, WI) as described previously (136). The proluminescent substrate containing the amino acid sequence Asp-Glu-Val-Asp (DEVD) is cleaved by activated caspase-3/7, leading to the release of a luciferase substrate (aminoluciferin) and the generation of luminescent signal. Data were presented as relative luminescent units ( $R L U)$ which were normalized to the corresponding values of control cells as an indicator of caspase $3 / 7$ activities. TUNEL labeling was carried out by the Pathology Research Services Laboratory at University of Washington. The slides were scanned by a ScanScope CS digital slide scanner (Aperio; Vista, CA) and analyzed by ImageJ software ( $\mathrm{NIH})$ as described previously (137). 


\subsubsection{Measuring $\Delta \psi$ mito using imaging microscopy}

Cells were incubated with $10 \mu \mathrm{M} \Delta \psi$ mito probe $\mathrm{JC} 1$ in growth media for 10 minutes at room temperature followed by three washes with Ringer's solution. Dye-loaded cells were mounted onto a chamber on the stage of a Nikon Diaphot inverted microscope with a Fluor 20x objective $(0.75 \mathrm{NA})$ at room temperature. Treatments were made by diluting stock solutions into Ringer's solution at the indicated concentrations. Fluorescence imaging measurements of $\Delta \psi$ mito were performed as reported previously $(93,138)$. Briefly, a charge coupled device camera collected JC-1 emission images using filter wheels (Lambda-10, Sutter Instruments, Novato, CA). Images were corrected for background (region without cells). Quantitative data are reported as JC1 fluorescence ratios normalized to minimal $\mathrm{JC} 1$ ratios obtained at the start of the experiment and maximal JC1 ratios obtained after $5 \mu \mathrm{M}$ FCCP treatment: Normalized JC1 Ratio $=($ Ratio at a given time - minimal Ratio) / (maximal ratio - minimal Ratio) * $100 \%$. Normalized $\mathrm{JC} 1$ ratios at plateau (before addition of FCCP) from different experiments have been averaged for summary charts.

\subsubsection{Immunofluorescence Microscopy}

For immunofluorescence staining of A549 and HCT116 cells, cells plated 24 hours earlier were incubated for 3 hours with either vehicle (DMSO) or $50 \mu \mathrm{M}$ C12 in Ringer's solution. The immunofluorescence staining of cytochrome c was carried out as described previously (134). Images were captured using a Nikon 
Eclipse Ti confocal microscope with a 40x CFI Plan Fluor objective (NA 0.6). To minimize variability, the same microscope settings were used for all experiments. For immunofluorescence staining of tumor sections, tumor sections $(5 \mu \mathrm{m})$ were treated with antigen retrieval procedure by boiling in $10 \%$ Triton $x-100$, then slowly cooled down at room temperature. After incubating with the blocking buffer ( $1 \times$ PBS, $0.2 \%$ Triton X-100, 5\% goat serum), the slides were incubated with antibodies against activated caspase-3 (Cell signaling) overnight at $4^{\circ} \mathrm{C}$. Following three 10-minute washes, slides were incubated with goat anti-rabbit IgG (Alexafluor-568, Invitrogen) for 1 hour. The fluorescence was visualized by confocal microscopy using a 40x CFI Plan Fluor objective (NA 0.6) and was analyzed using ImageJ software (NIH) as described previously (139).

\subsubsection{In vivo animal studies}

For C12 toxicity studies, DMSO or C12 (25 mg/kg/day) was administered intraperitoneally each day in 8 week-old C57BL/6 mice or in 6 week-old athymic nude mice. All organs were collected and weighted at sacrifice.

For transplanted tumors in C57BL/6 mice, eight-week old C57BL/6 female mice (Jackson Laboratories; Bar Harbor, ME) were inoculated subcutaneously (s.c.) with $1 \times 10^{6}$ Lewis Lung Carcinoma cells on the right flank. Tumors were measured daily with dull edged Vernier calipers $\left(\mathrm{V}=\mathrm{L} \times \mathrm{W}^{2} / 2\right)$. After tumor size reached around $100 \mathrm{~mm}^{3}$, animals with size-matched tumors were divided into control group and $\mathrm{C} 12$ group. DMSO or $\mathrm{C} 12$ was administered intraperitoneally 
each day. At the end of the experiments, tumors were excised for apoptosis evaluation. For the study of transplanted human tumors, female athymic nude mice (5-7 weeks) were purchased from Harlan Laboratories (Indianapolis, IN). Wild-type HCT116 cells and Bax/Bak DKO HCT116 cells $\left(3.0 \times 10^{7} / \mathrm{ml}\right)$, wild-type A549 cells and Bcl-2-overexpressing A549 cells $\left(3.0 \times 10^{7} / \mathrm{ml}\right)$ were resuspended and mixed 1:1 (vol/vol) with Matrigel (BD Biosciences), then $0.1 \mathrm{ml}$ mixture of cells and Matrigel was injected s.c. on the right flank of mice. All animals with size-matched tumors were divided into two groups (control and C12) when the tumor size reached around $100 \mathrm{~mm}^{3}$. For HCT116 and Bax/Bak DKO HCT116 tumors, DMSO or C12 was administered intraperitoneally each day. DMSO or etoposide (40 mg/kg/day) was administered intraperitoneally once every other day. For wild-type A549 tumors, animals were treated with $\mathrm{C} 12$ for 6 times/week. For Bcl-2 over-expressing A549 cells tumors, DMSO or C12 was administered with $\mathrm{C} 12$ each day. At the end of the experiments, tumors were excised for apoptosis evaluation.

\subsubsection{Statistical analysis}

Results are presented as mean \pm standard deviation of at least three independent experiments. Statistical analysis was performed using Student's two-tail t-test. A p value $<0.05$ was considered significant. 


\subsection{RESULTS}

3.3.1. C12 inhibits lung tumor growth and induces tumor cell apoptosis in vivo. The cytotoxic effects of $\mathrm{C} 12$ on tumor cells have been reported previously (66, 69-71, 140), but whether they are selective for transformed cells was unknown. To investigate whether oncogenic transformation influences the cytotoxicity of C12, we studied normal human bronchia/tracheal epithelial (NHBE) and corresponding HBE immortalized and transformed successively by telomerase, SV40 large T antigen and activated Ras (H-ras V12) (141-144). This is a wellestablished epithelial cell malignant transformation system related to human lung cancer. Following C12 treatment, transformed HBE exhibited higher levels of cell death (Figure 3.1A) and caspase-3/7 activation (Figure 3.1B) compared with their untransformed counterparts, indicating that $\mathrm{C} 12$ induces apoptosis preferentially in transformed cells.

To investigate the relevance of $\mathrm{C} 12$ cytotoxicity on transformed cells to tumor growth in animals, we examined the effects of $\mathrm{C} 12$ on the growth of established Lewis Lung Carcinoma (LLC) tumors. As shown in Figure 1C, transplanted tumors grew much more slowly in C12-treated mice than in vehicle-treated mice, revealing a dose-dependent anti-tumor activity of $\mathrm{C} 12$ as a single agent.

To test the involvement of apoptosis in tumor growth inhibition, established LLC tumors from mice were analyzed for caspase-3 activation through immunofluorescence staining (Figures 3.1D-E), western blot (Figures 3.1F-G) and TUNEL staining (Figures 3.1H-I). The percentage of TUNEL-positive cells 
and the levels of activated caspase-3 were higher in tumors from C12-treated mice than in those of the control group, suggesting that apoptosis is involved in the inhibitory activity of $\mathrm{C} 12$ in vivo. 

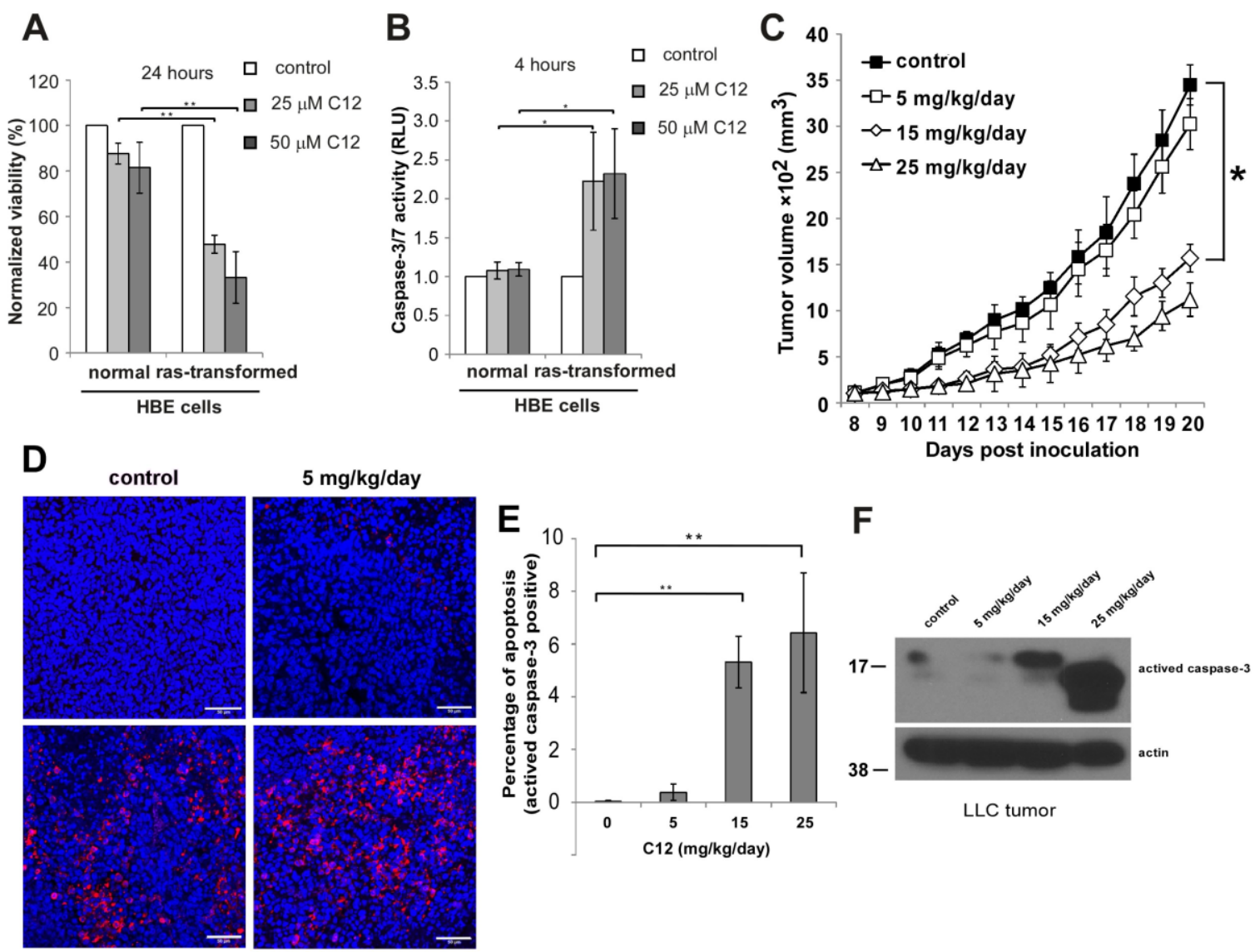

$15 \mathrm{mg} / \mathrm{kg} / \mathrm{day}$

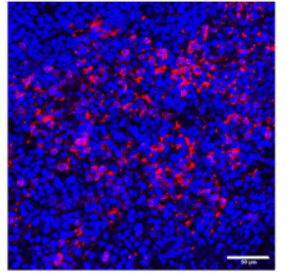

E

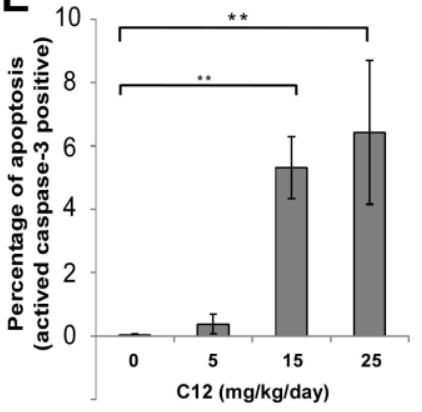

F

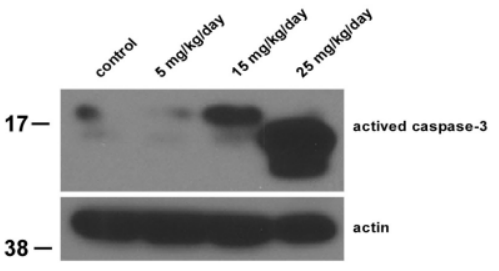

LLC tumor

$25 \mathrm{mg} / \mathrm{kg} / \mathrm{day}$ activated caspase-3

G

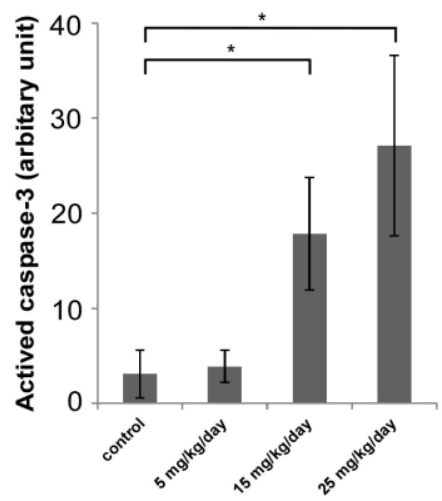

H

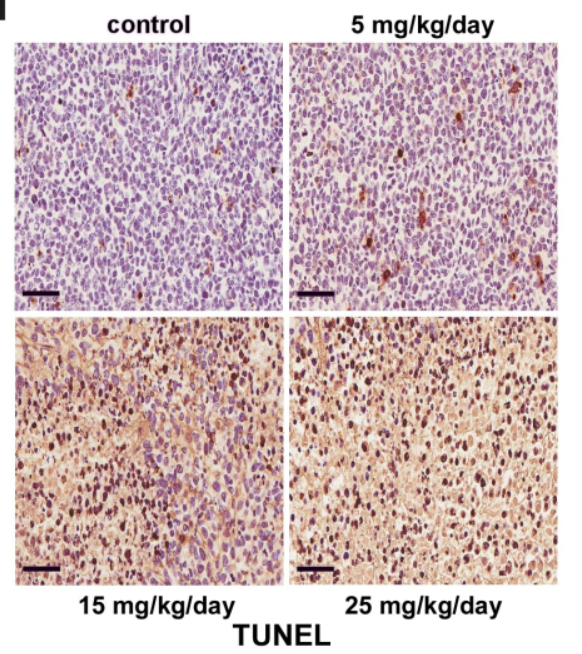

I

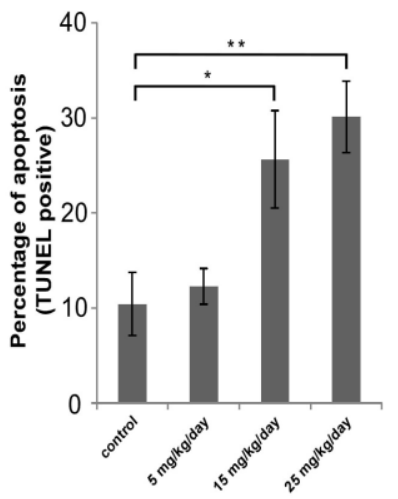


Figure 3.1. C12 inhibits LLC tumor growth and induces tumor cell apoptosis in vivo in a dose-dependent fashion.

(A-B) Cytotoxicity of C12 is affected by oncogenic transformation. C12's effects on HBE cell viability (A) and caspase-3/7 activation (B) were examined. All data shown are mean \pm standard deviation of 3 independent experiments. Asterisk indicates $\mathrm{P}<0.05\left(^{*}\right)$ or $\mathrm{P}<0.01\left(^{* *}\right)$ by student's unpaired $t$ test. $(\mathrm{C})$ The inhibitory effects of $\mathrm{C} 12$ on the growth of LLC tumors were studied. Tumors were measured daily and tumor tissues were removed at the end of treatments. Data are shown as mean \pm standard deviation of tumor volumes of 7 animals in either vehicle control or $\mathrm{C} 12$-treated group. Asterisk indicates $\mathrm{P}<0.05\left(^{*}\right)$ by student's unpaired $\mathrm{t}$ test. (D) Apoptotic cells in tumor sections were detected by immunofluorescence staining of activated caspase-3. Representative images of tumor sections are shown. Scale bar, $50 \mu \mathrm{m}$. (E) The percentage of activated caspase-3 shown in (D) was quantified using ImageJ software $(\mathrm{NIH})$. Data are mean \pm standard deviation of three independent tumor sections. Asterisk indicates $\mathrm{P}<0.01\left(^{* *}\right)$ by student's unpaired t test. (F) Expression of activated caspase-3 in tumor tissues was analyzed by western blot. (G) The relative expression levels of activated caspase3 shown in $(F)$ were quantified by measuring intensities of western blot signals using Image J software and presented as arbitrary units. Data are mean \pm standard deviation of three independent tumor samples. Asterisk indicates $\mathrm{P}<0.05\left(^{*}\right)$ by student's unpaired t test. $(\mathrm{H})$ TUNEL staining of apoptotic cells in control or C12treated tumor sections. Representative images are shown. Scale bar, $60 \mu \mathrm{m}$. (I) The percentage of apoptotic cells shown in $(\mathrm{H})$ was quantified using ImageJ 
software. Data are mean \pm standard deviation of three independent tumor sections. Asterisk indicates $\mathrm{P}<0.05\left(^{*}\right)$ or $\mathrm{P}<0.01\left(^{* *}\right)$ by student's unpaired t test. 
3.3.2. C12 does not cause significant toxicity in mice.

To examine whether $\mathrm{C} 12$ possesses non-specific toxicity to animals, we investigated the effects of administrating $\mathrm{C} 12$ to mice for extended periods of time. No significant changes in body weight and organ weight (spleen, kidney, liver, heart, lung) were observed for C12-treated C57BL/6 mice, showing no evidence of significant toxicity of $\mathrm{C} 12$ upon administration (Figures 3.2A and 3.2C). Similarly, C12 caused no significant change in body weight and organ weight (spleen, kidney, liver, heart and lung) in athymic nude mice (Figures 3.2B and 3.2D). 
A

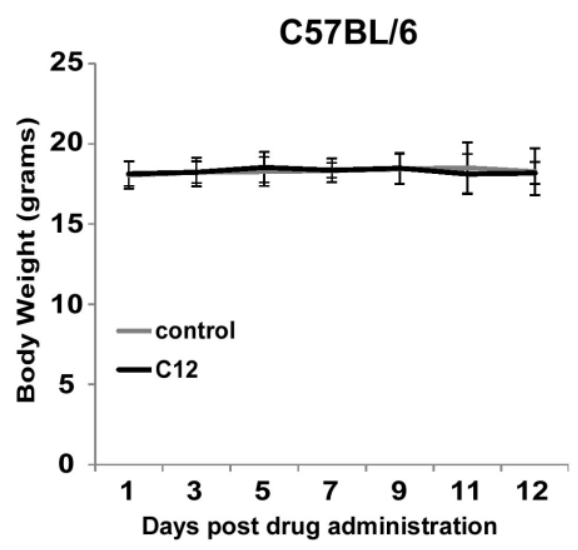

C

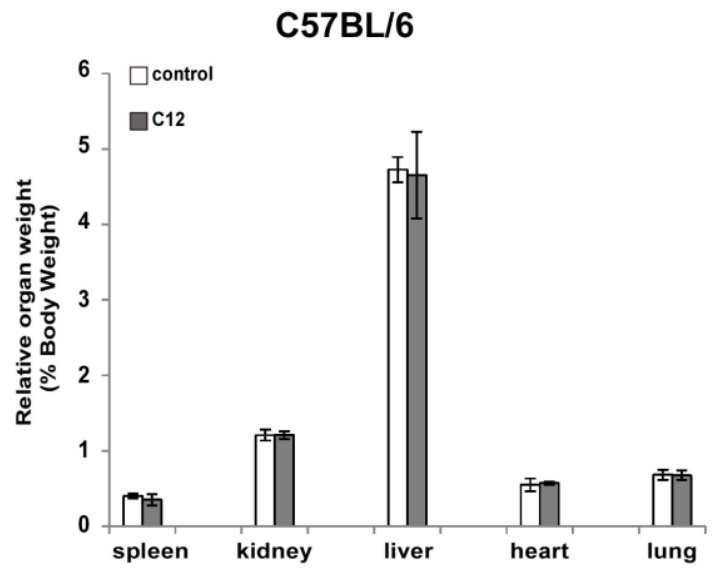

B

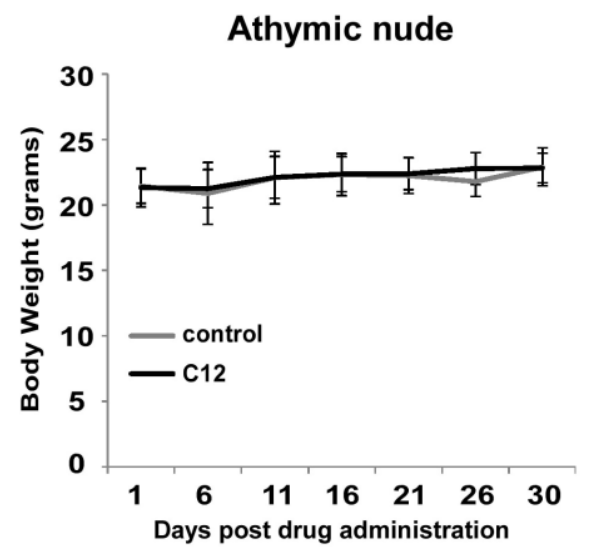

D

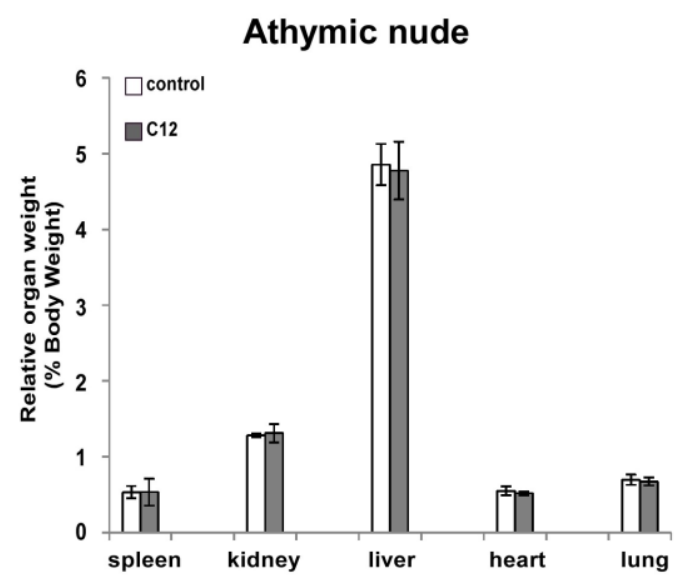


Figure 3.2. Administrating $\mathrm{C} 12$ to mice does not affect body weight and organ weight.

(A-B) DMSO or C12 (25 mg/kg/day) was administered intraperitoneally to C57BL/6 mice $(A)$ or athymic nude mice $(B)$ daily. Body weights of mice were measured. Data are mean \pm standard deviation of body weights of 5 animals in each group. (C-D) Effects of $\mathrm{C} 12$ on organ weights in C57BL/6 mice (C) and athymic nude mice (D) were evaluated. Spleen, kidney, liver, heart and lung of the indicated mice were weighed at sacrifice. Each organ weight was normalized as the percentage of corresponding body weight. Data are mean \pm standard deviation of organ weights of 5 animals in each group. 
3.3.3. C12 and conventional therapeutic drugs induce tumor cell death synergistically.

In order to explore the therapeutic potential of $\mathrm{C} 12$, we studied the combinatorial effects of $\mathrm{C} 12$ and therapeutic drugs. $\mathrm{A} 549$ and $\mathrm{NCl}-\mathrm{H} 1299$ cells were treated with various concentrations of $\mathrm{C} 12$ and $\mathrm{ABT}-737$, an inhibitor of $\mathrm{Bcl}-2$ and $\mathrm{BCl}-\mathrm{XL}$, at a constant dose ratio. Cell death was measured at the indicated times, and the combination index $(\mathrm{Cl})$ values were determined (Figure 3.3). As shown in figure $3 \mathrm{C}$ and $3 \mathrm{~F}$, in the range of tested drug concentrations, $\mathrm{Cl}$ values were smaller than 1, indicating that C12 and ABT-737 exhibited synergistic cytotoxic effects on A549 cells and $\mathrm{NCl}-\mathrm{H} 1299$ cells. 
A

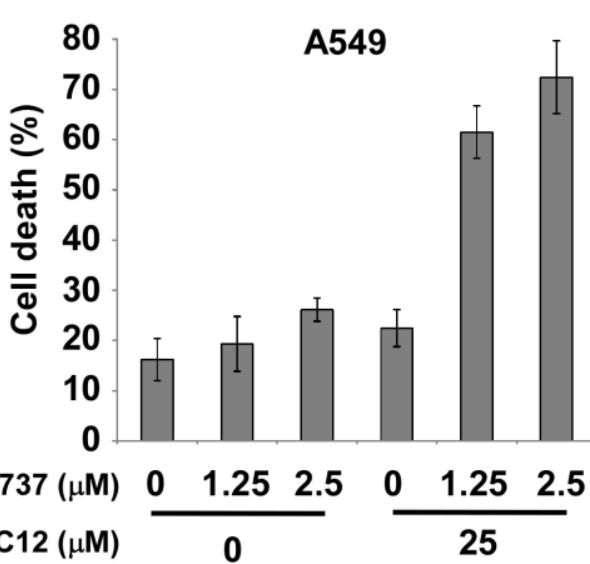

C

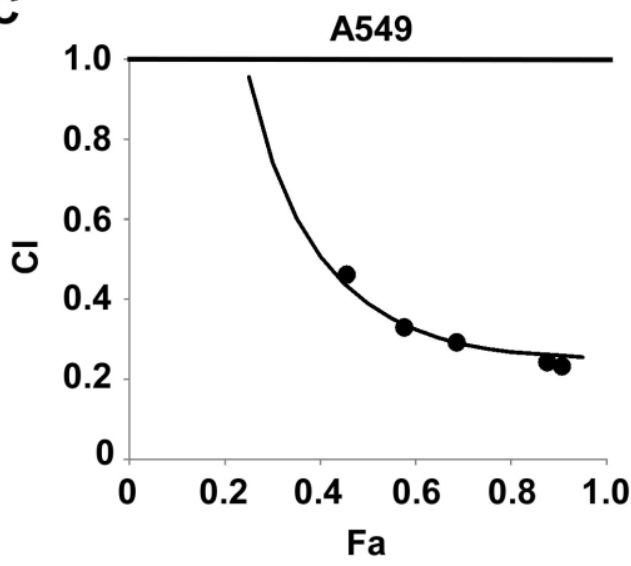

E

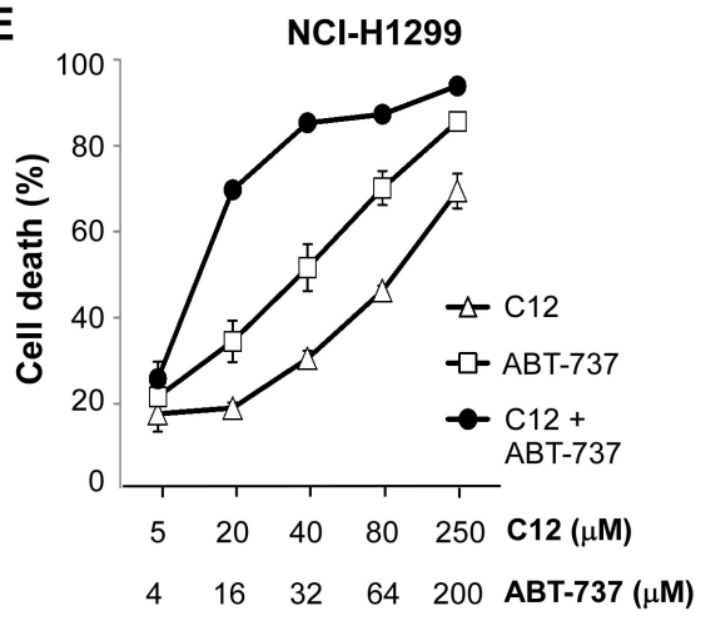

B

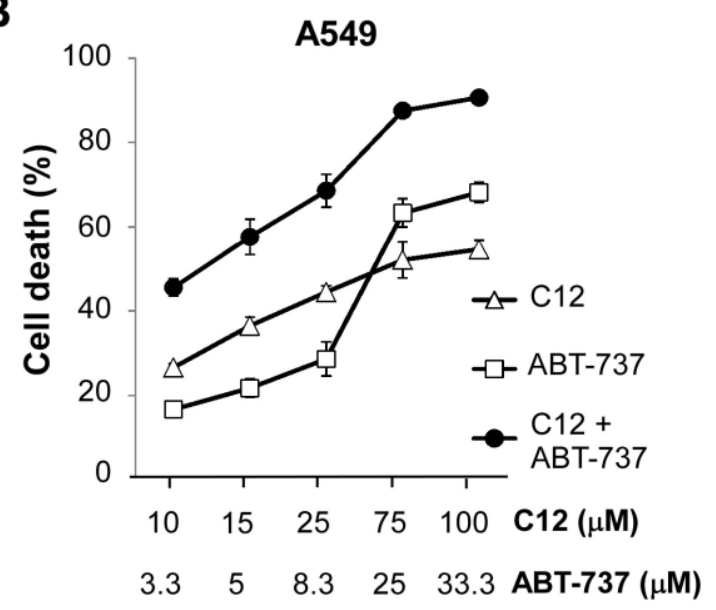

D

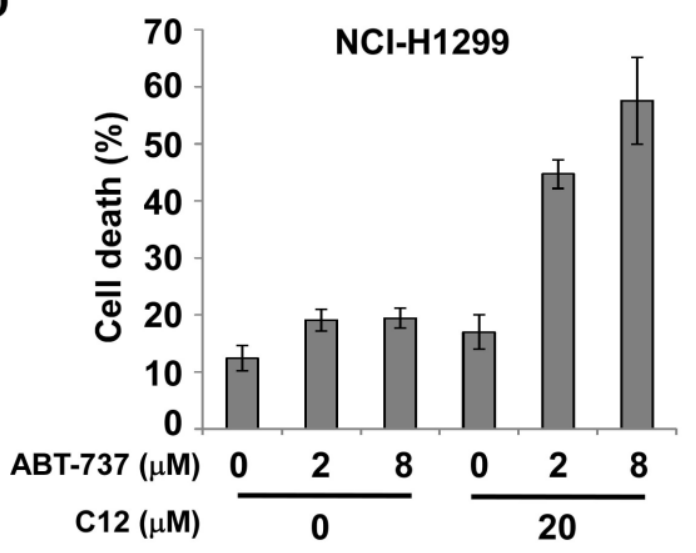

F

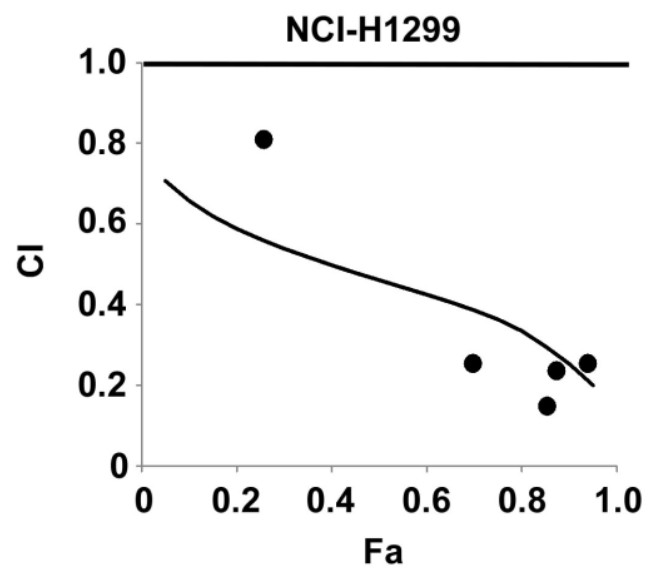


Figure 3.3. C12 functions synergistically with ABT-737 to induce tumor cell death. (A) C12 sensitized A549 cells to ABT-737 treatment. Upon treatment with different combinations of $\mathrm{C} 12$ and $\mathrm{ABT}-737$ for 48 hours, the percentage of cell death of A549 cells was measured. The data depict mean \pm standard deviation of three independent experiments. (B) A549 cells were treated with the indicated combination of $\mathrm{C} 12(10-100 \mu \mathrm{M})$ and ABT-737 (3.3-33.3 $\mu \mathrm{M})$ for 48 hours, and cell death was measured. The data represent mean \pm standard deviation of triplicate experiments. The experiments were performed independently three times. (C) The data shown in (B) was analyzed as described in MATERIALS AND METHODS. The values of combination index $(\mathrm{Cl})$ and fractional activity $(\mathrm{Fa})$ were calculated. (D) $\mathrm{NCl}-\mathrm{H} 1299$ cells were treated with different combinations of $\mathrm{C} 12$ and ABT-737 for 48 hours, and cell viability was measured. The data are mean \pm standard deviation of three independent experiments. (E) NCl-H1299 cells were cultured with the fixed combination of C12 $(5-250 \mu \mathrm{M})$ and ABT-737 $(4-200 \mu \mathrm{M})$ for 48 hours, and cell death was determined. The data are mean \pm standard deviation of triplicate experiments. The experiments were performed independently three times. $(F)$ The data shown in $(E)$ was evaluated as described in MATERIALS AND METHODS and $\mathrm{Cl}$ values were computed. 
3.3.4. Anti-apoptotic Bcl-2 proteins do not affect $\mathrm{C} 12$ cytotoxicity.

Anti-apoptotic Bcl-2 proteins are frequently overexpressed in human cancers and associated with chemotherapeutic resistance and relapse (145). To investigate the involvement of anti-apoptotic Bcl-2 proteins in $\mathrm{C} 12$-induced human tumor cell apoptosis, Bcl-2, Mcl-1 and Bcl-XL were stably overexpressed in A549 cells respectively by retroviral infection (Figures 3.4A, 3.5A and 3.5D). The anti-tumor drug actinomycin D caused less cell death and caspase-3/7 activation in Bcl-2-, Mcl-1- or Bcl-xL-overexpressing cells than in cells expressing the empty vector (Figures 3.4B-C, 3.5B-C and 3.5E-F). In contrast, $\mathrm{C} 12$ induced similar levels of cell death and caspase-3/7 activation in cells overexpressing Bcl-2, Mcl-1 or Bcl$\mathrm{X} L$ and the vector control cells (Figures 3.4B-C, 3.5B-C and 3.5E-F). To further explore the involvement of $\mathrm{Mcl}-1$ in mediating $\mathrm{C} 12$-induced cytotoxicity, we examined A549 cells in which Mcl-1 expression was stably reduced by shRNA (146). Actinomycin D but not C12 caused more cell death and caspase-3/7 activation in Mcl-1-knockdown cells than the cells expressing the control scramble shRNA (Figures 3.5G-H), further validating that Mcl-1 expression is not vital for $\mathrm{C} 12$-induced apoptotic signaling.

MOMP has been recognized to be a "no-return" step during mitochondria-based apoptotic signaling (105). To further investigate C12-initiated apoptosis in tumor cells, we studied the involvement of Bcl-2 in two key events of MOMP: depolarization of mitochondrial membrane potential ( $\Delta \psi$ mito $)$ and cytochrome $\mathrm{C}$ release. Depolarization of $\Delta \psi$ mito was evaluated by determining the changes in 
fluorescence of the voltage-dependent dye $\mathrm{JC} 1$ released from mitochondria into the cytosol and nucleus. Within minutes of C12 exposure, mitochondria in A549vector cells and A549-Bcl-2 overexpressing cells were depolarized to the same degree (Figures 3.4D-E). Moreover, C12 caused cytochrome c release from mitochondria in both A549-vector and Bcl-2 or Mcl-1 over-expressing A549 cells (Figures 3.6 and 3.7). Taken together, these results indicate that overexpression of anti-apoptotic $\mathrm{Bcl}-2$ proteins reduces apoptosis in response to the classical anti-tumor drug actinomycin $\mathrm{D}$ but fails to provide protection against $\mathrm{C} 12$. 
A

B

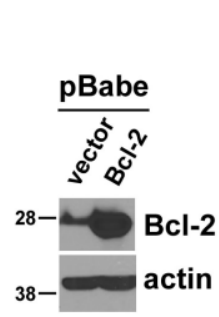

C

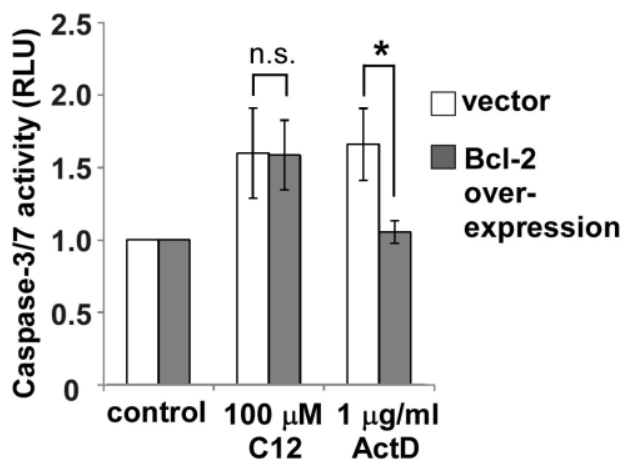

D

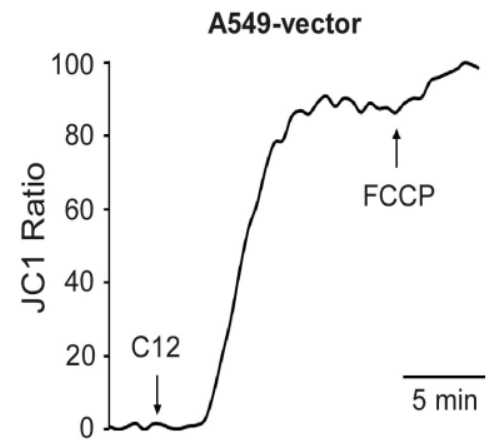

E

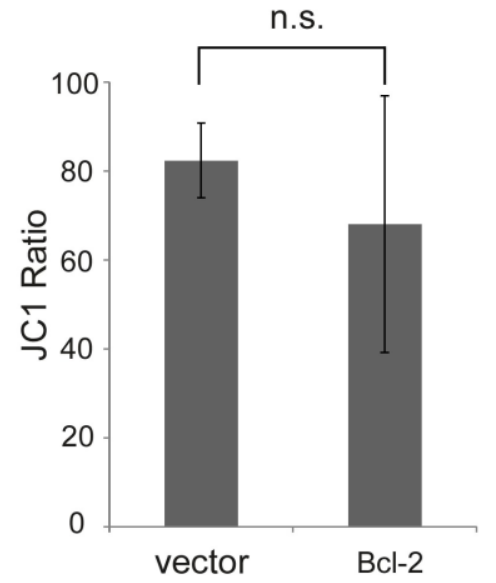

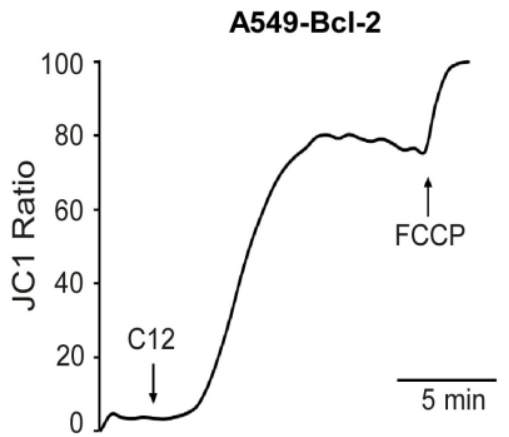


Figure 3.4. C12 induces tumor cell apoptosis independent of anti-apoptotic protein Bcl-2.

(A) Retrovirally overexpressed Bcl-2 in A549 cells was examined by western blot. (B) Cell viability was measured 48 hours after C12 or actinomycin D (ActD) exposure. (C) Caspase-3/7 activities were determined following 2 hour exposure to C12 and 24 hour exposure to actinomycin D. (D) C12's effect on mitochondrial membrane potential is independent of Bcl-2. A549-vector and A549-Bcl-2overexpressing cells were loaded with $\mathrm{JC} 1$, and its fluorescence was measured using imaging microscopy during the treatment with $100 \mu \mathrm{M} \mathrm{C} 12$ and $5 \mu \mathrm{M} \mathrm{FCCP}$. Typical results from three independent experiments are shown. (E) C12 caused equivalent depolarization of mitochondrial potential in vector and Bcl-2overexpressing A549 cells. All data are shown as mean \pm standard deviation of three independent experiments. Asterisks indicate $\mathrm{P}<0.05\left(^{*}\right)$; ns, no significant by student's unpaired t test. 
A

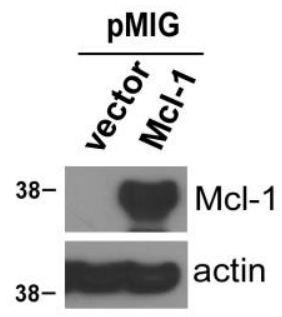

B

E
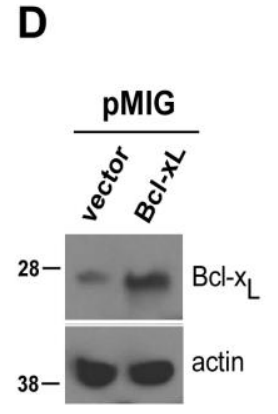

38-
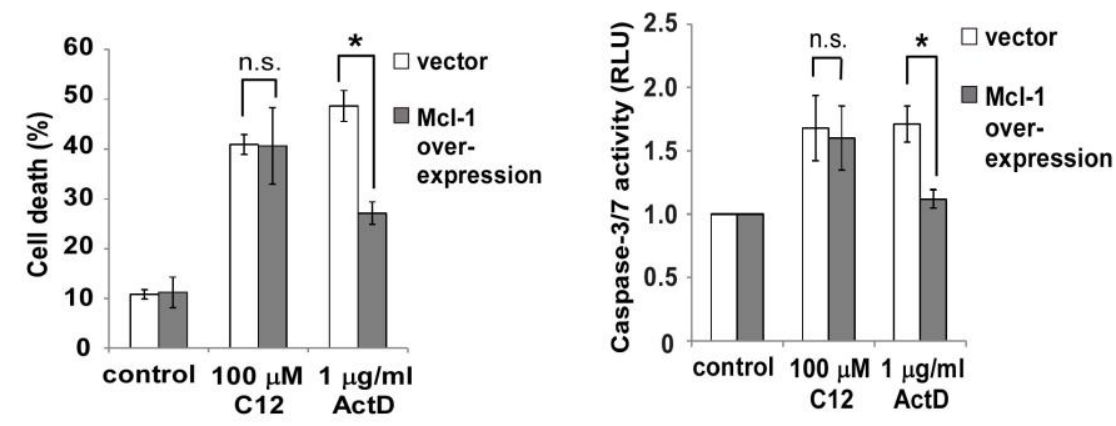
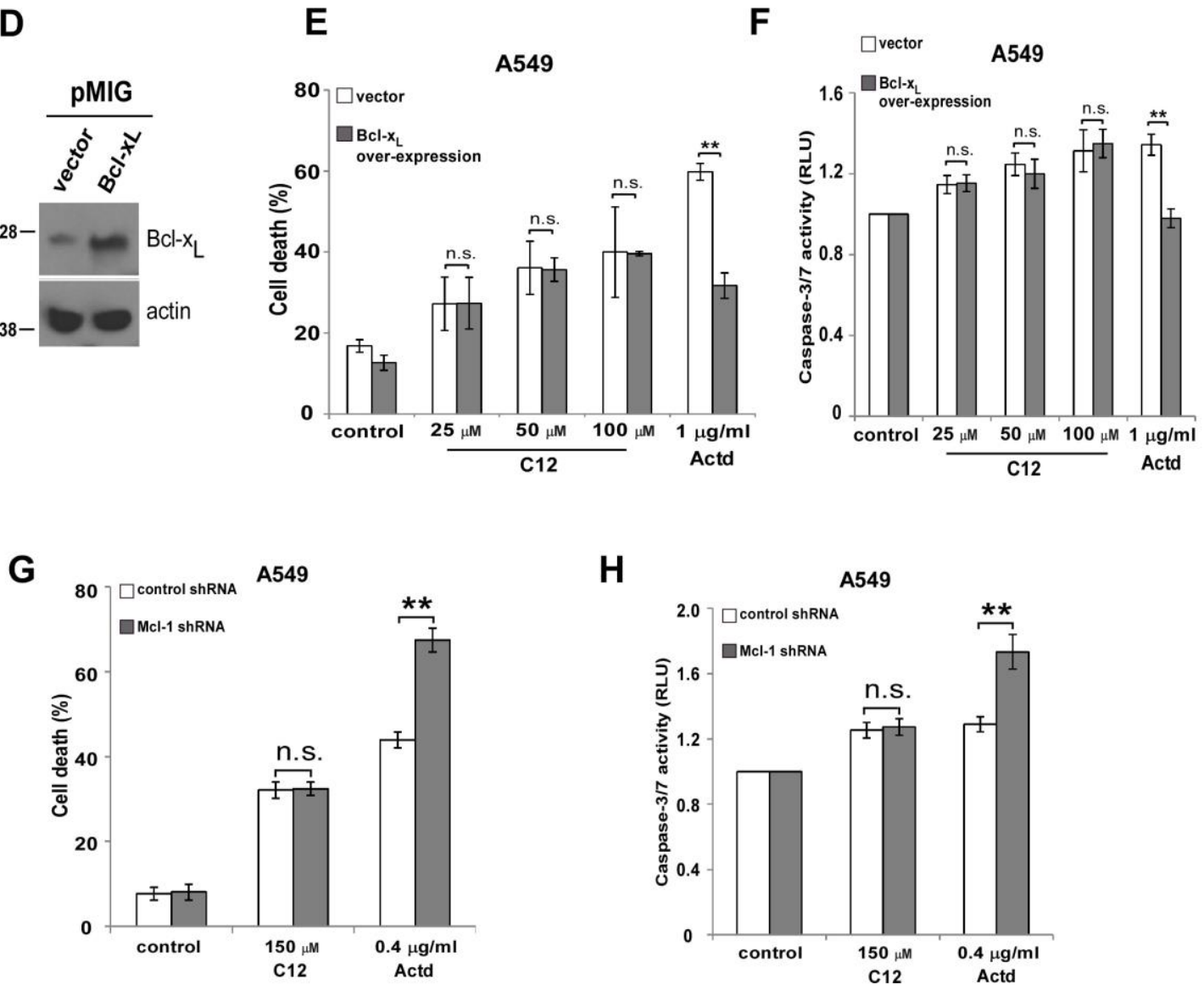
Figure 3.5. Anti-apoptotic Bcl-2 protein $\mathrm{Mcl}-1$ and $\mathrm{Bcl}-\mathrm{xL}$ do not affect $\mathrm{C} 12$ cytotoxicity.

(A)The expression levels of Mcl-1 in the indicated A549 cells were determined by western blot. (B) Cell viability of the indicated A549 cells with or without Mcl-1 over-expression was measured 48 hours after the treatment. (C) Caspase-3/7 activities were determined after exposure to $\mathrm{C} 12$ for 2 hours and exposure to actinomycin D for 24 hours. (D) Bcl-xL was stably overexpressed in A549 cells by retroviral infection. (E) Cell death was measured after 48 hour-incubation of $\mathrm{C} 12$ or actinomycin D $(1 \mu \mathrm{g} / \mathrm{ml})$ in A549 cells without or with over-expressed Bcl-XL. (F) Caspase-3/7 activities were examined 32 hours after $C 12$ or actinomycin D exposure. (G) After treating A549 cells expressing Mcl-1 shRNA or control shRNA with $\mathrm{C} 12$ or actinomycin D for 72 hours, cell death was measured. $(\mathrm{H})$ Caspase$3 / 7$ activities were measured after 32 hour-incubation of $C 12$ or actinomycin $D$ in the indicated A549 cells. All data are presented as mean \pm standard deviation of three different experiments that were carried out in triplicate. Asterisk indicates $\mathrm{P}<$ $0.05\left(^{*}\right)$ or $\mathrm{P}<0.01\left(^{* *}\right)$ by student's unpaired t test. ns, no significant. 


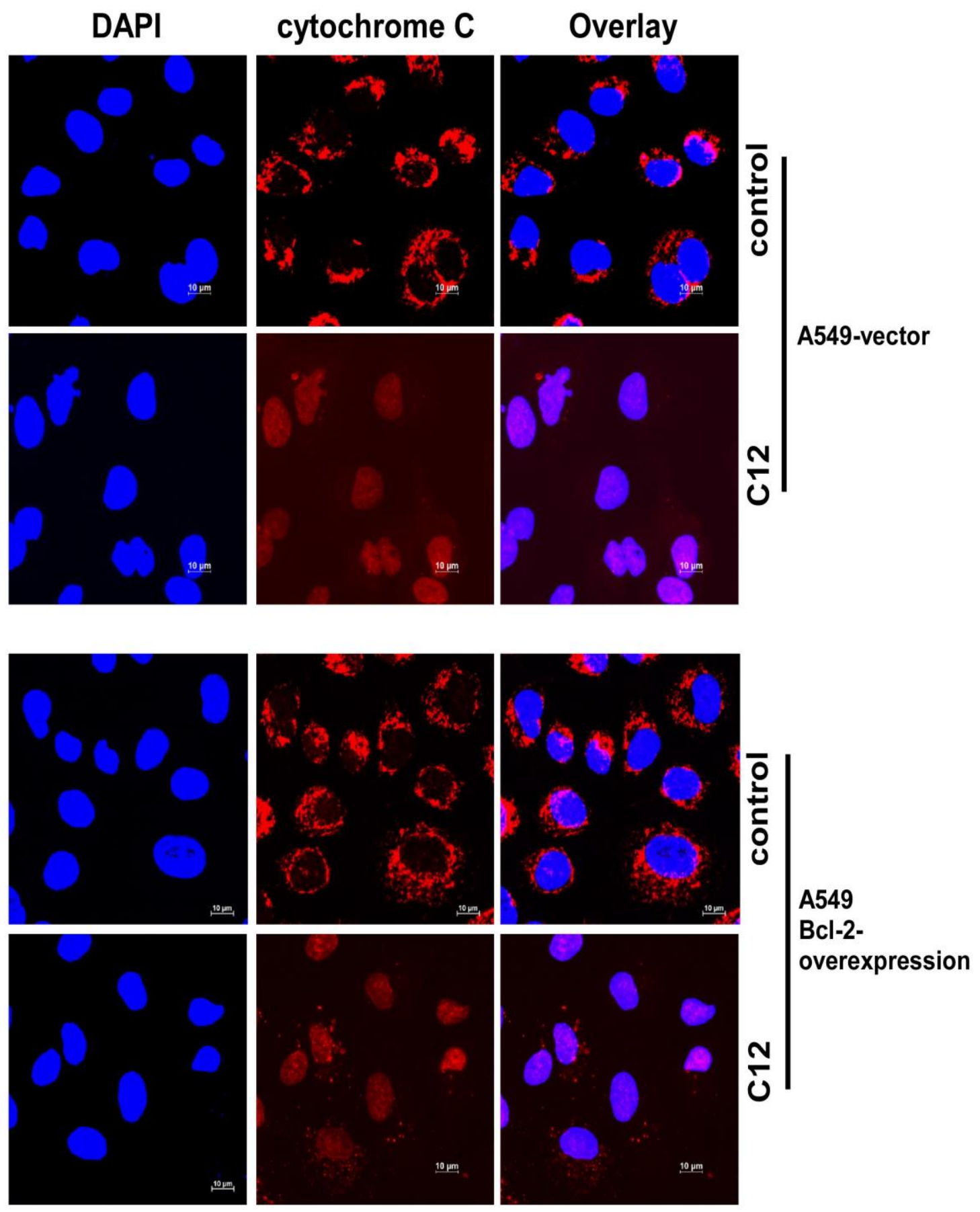


Figure 3.6. C12-induced cytochrome $c$ release in tumor cells is independent of Bcl-2.

Upon C12 exposure, cytochrome $\mathrm{c}$ is released from mitochondria in both A549vector and A549-Bcl-2 over-expressing cells. Representative confocal images of A549 cells treated with either DMSO (control) or $100 \mu \mathrm{M}$ C12 for 3 hours.

Cytochrome $\mathrm{c}$ is shown in red, and DAPI is shown in blue. Following $\mathrm{C} 12$ treatment, staining of cytochrome c became diffuse. Scale bar, $10 \mu \mathrm{m}$. 

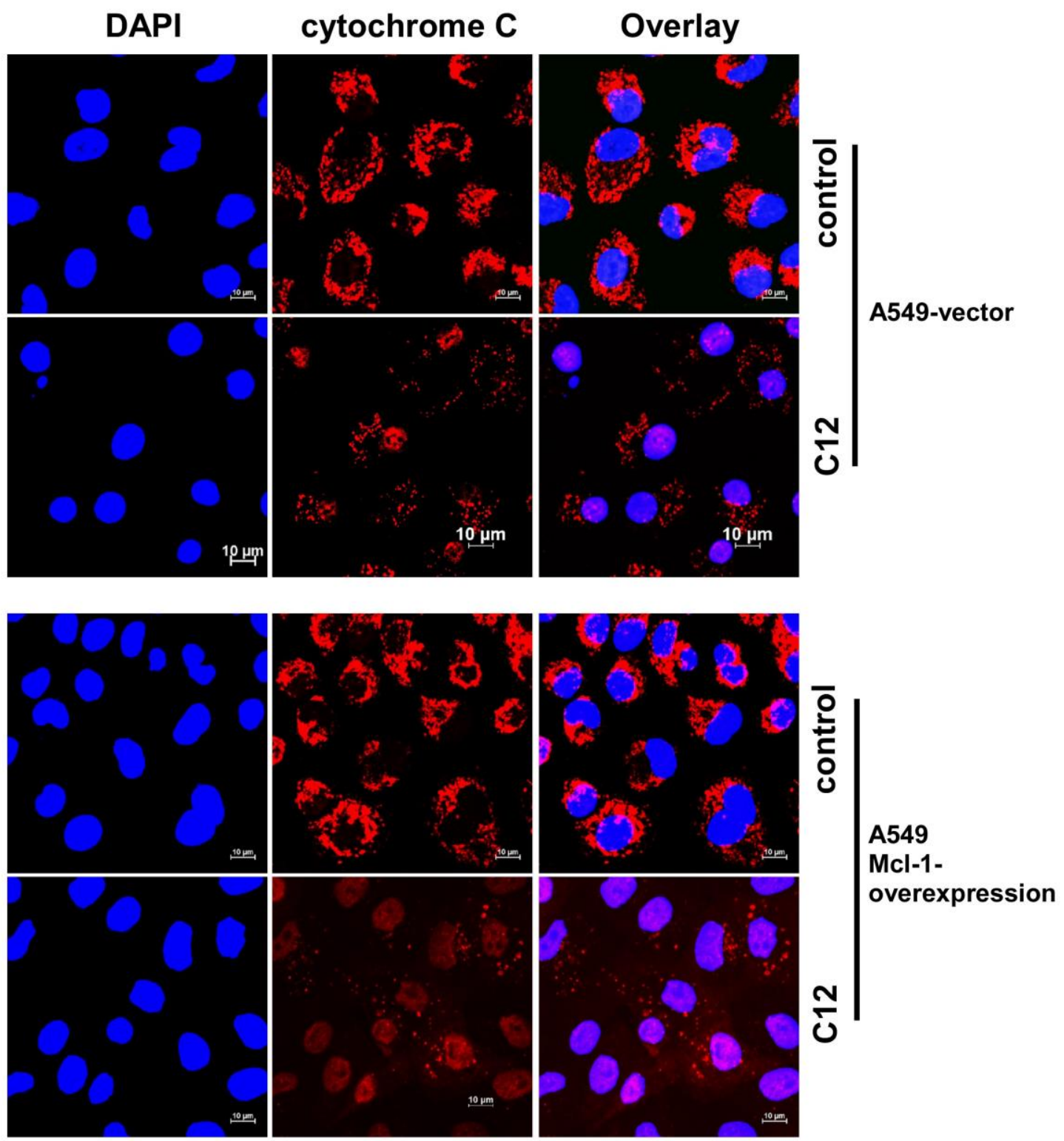
Figure 3.7. C12-induced cytochrome c release in tumor cells is independent of Mcl-1.

After treating with $100 \mu \mathrm{M} \mathrm{C12}$ of 3 hours, cytochrome $\mathrm{c}$ was released from mitochondria in both A549-vector and A549-Mcl-1 over-expressing cells.

Representative images acquired by confocal microscopy are shown. Scale bar, $10 \mu \mathrm{m}$. 
3.3.5. C12 blocks tumor growth independent of $\mathrm{Bcl}-2$ protein.

To determine whether anti-apoptotic Bcl-2 protein-independent $\mathrm{C} 12$ cytotoxicity is relevant in vivo, we examined its effects on the growth of tumors originated from A549 cells with different levels of Bcl-2 expression in athymic nude mice. As shown in Figures 3.8A-B, A549-Bcl-2 overexpressing tumors grew much faster than their vector control counterparts, which is consistent with the oncogenic function of Bcl-2 (147). C12 inhibited the growth of vector control A549 tumors and Bcl-2-overexpressing A549 tumors to a comparable degree. Inhibitory effects of $\mathrm{C} 12$ on tumor growth are likely attributed to apoptosis, as more TUNELpositive cells were detected in C12-treated tumors than vehicle-treated ones regardless of Bcl-2 expression levels (Figures 3.8C-D). Overall, these results show that $\mathrm{Bcl}-2$ is not involved in the cytotoxic effects of $\mathrm{C} 12$ in vitro as well as in vivo. 
A

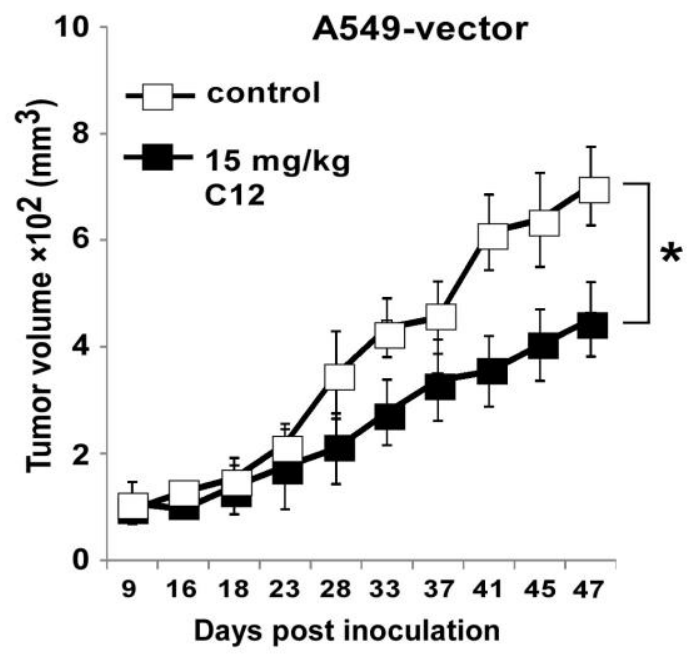

C

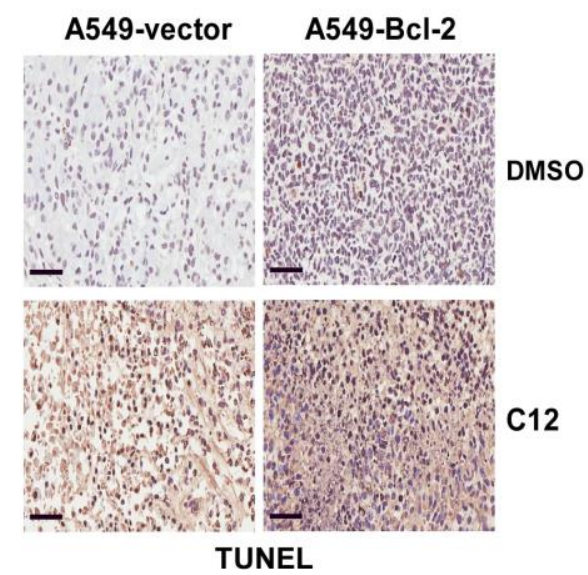

B

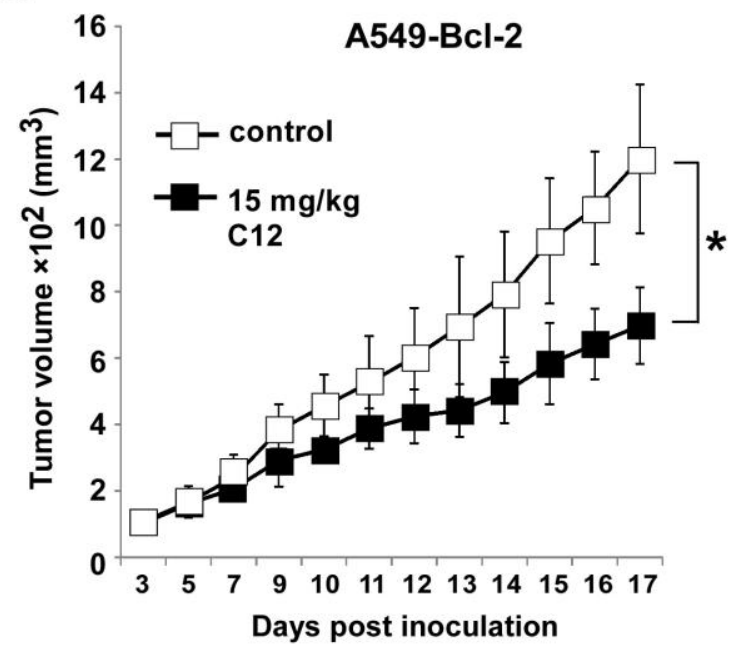

D

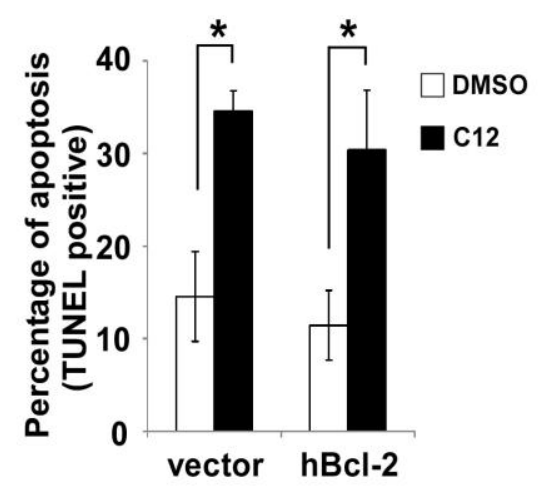


Figure 3.8. C12 inhibits tumor growth independent of Bcl-2.

(A-B) Growth of A549-vector (A) and A549-Bcl-2-overexpressing tumors (B) in athymic nude mice treated with vehicle control or C12 (15 mg/kg/day). Data are shown as mean \pm standard deviation of tumor volumes of 8 animals in either vehicle control (DMSO) or C12-treated groups. (C) Apoptotic cells in tumor sections were identified by TUNEL assay. Representative images of tumor sections from DMSO- and C12-treated mice are shown. Scale bar, $60 \mu \mathrm{m}$. (D) Summary of the data shown in (C). More apoptotic cells were detected in tumors of C12-treated mice than those of vehicle control mice. Mean \pm standard deviation of three independent tumor sections. For all the data, asterisk indicates $\mathrm{P}<0.05$ $\left(^{\star}\right)$ by student's unpaired $t$ test. 
3.3.6. Bak and Bax are not involved in C12-induced apoptosis.

Previous studies show that $\mathrm{C} 12$ induces apoptosis in MEFs independent of Bak and $\mathrm{Bax}(98,114)$, two pro-apoptotic Bcl-2 members required for MOMP in almost all apoptotic paradigms (148). To clarify their role in C12-induced tumor cell apoptosis, human colon carcinoma HCT116 cell lines lacking Bak alone, Bax alone, or both Bak and Bax (Bak/Bax-DKO) were studied (Figure 3.9A). C12 caused roughly equivalent cell death and caspase-3/7 activation in all the HCT116 cell lines examined, indicating that deficiency of Bak and Bax in HCT116 cells did not influence their responses to C12 (Figures 3.9B-C). Unlike C12, the therapeutic drug etoposide induced significant cell death and caspase3/7 activation in WT, Bax-KO and Bak-KO but not in Bak/Bax-DKO HCT116 cells (Figure 3.10A-B). Moreover, deficiency in Bak/Bax expression did not affect C12's effect to depolarize $\Delta \psi$ mito (Figures 3.9D-E) and release cytochrome c (Figure 3.11), indicating Bak/Bax are not involved in C12- induced MOMP in tumor cells. These results demonstrate that $\mathrm{C} 12$-induced apoptotic signaling is distinct from that triggered by conventional anti-cancer drugs. 


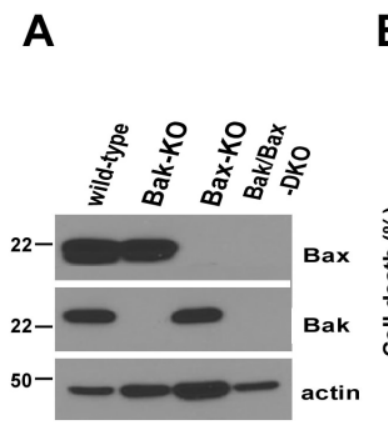

B

C
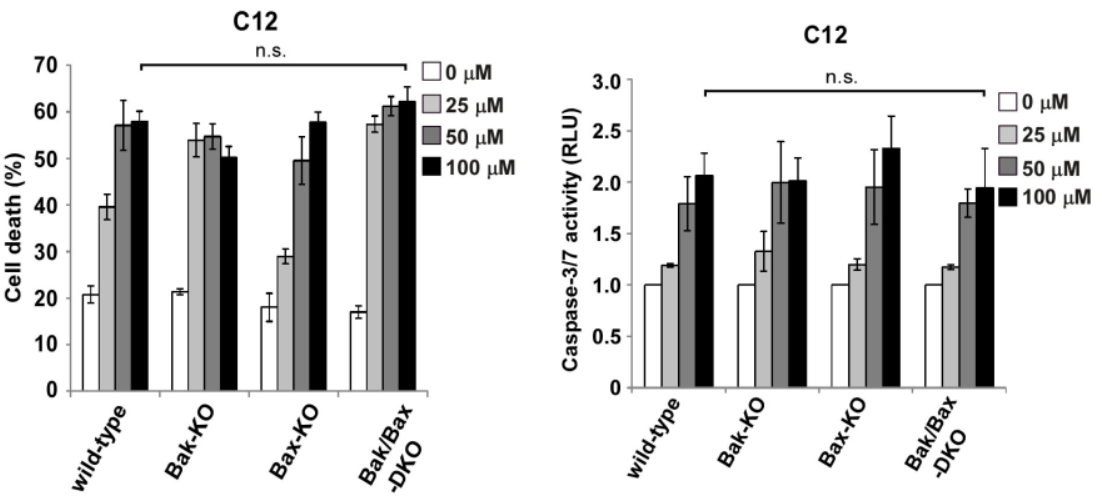

D

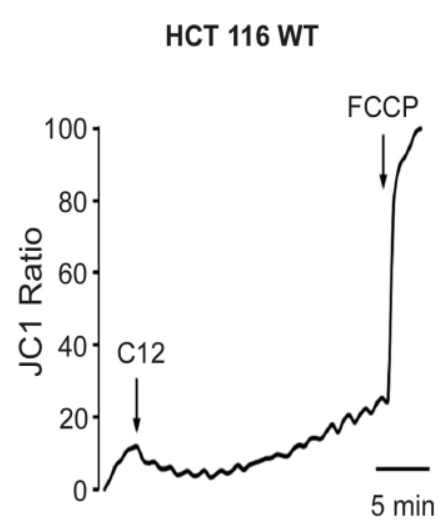

HCT 116 Bak/Bax DKO

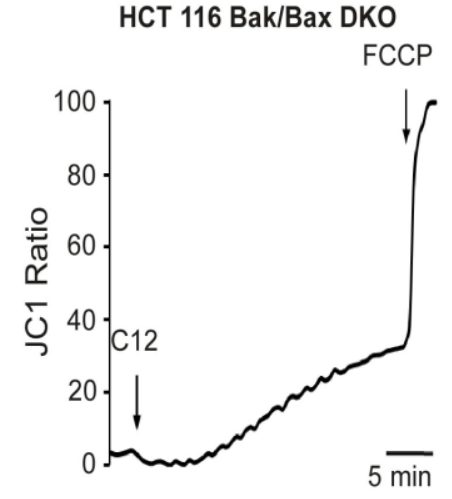

E

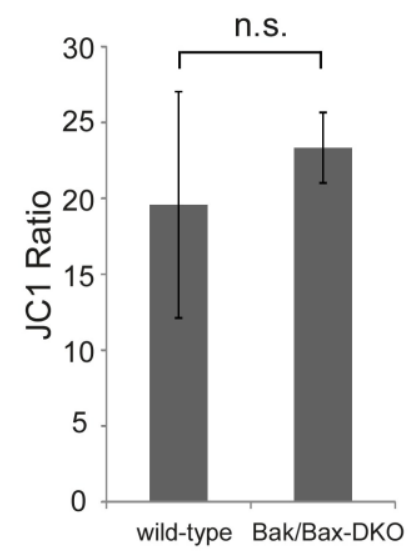


Figure 3.9. C12-induced tumor cell apoptosis is independent of Bak and Bax. (A) Bak and Bax expression in the indicated HCT116 cells was examined by western blot. (B-C) C12 induced similar levels of cell death (B) and caspase-3/7 activation (C) among WT, Bak-KO, Bax-KO and Bak/Bax-DKO HCT116 cells after 24 hours treatment. (D) The mitochondrial potential of the WT and Bak/Bax-DKO HCT116 cells loaded with JC1 was determined by fluorescent microscopy upon the treatment with $50 \mu \mathrm{M} \mathrm{C12}$ and $5 \mu \mathrm{M} \mathrm{FCCP.} \mathrm{Representative} \mathrm{results} \mathrm{are} \mathrm{shown.}$ (E) Summary of the data shown in (D). All data are presented as mean \pm standard deviation of three different experiments. ns, no significant. 
A
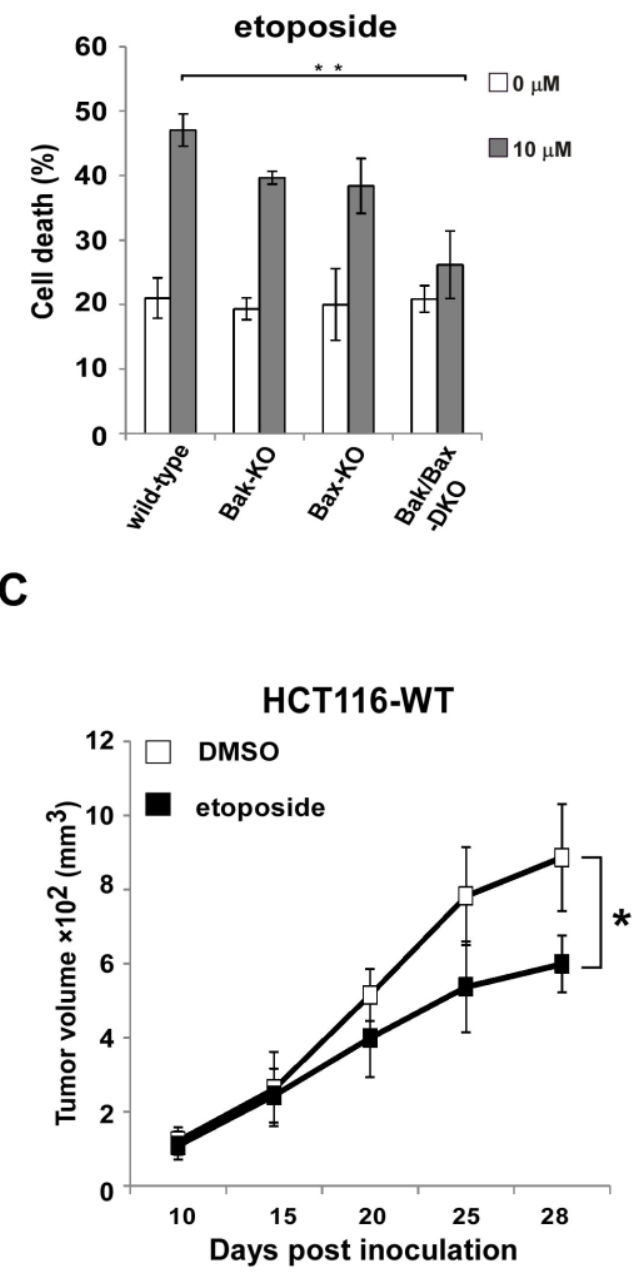

B

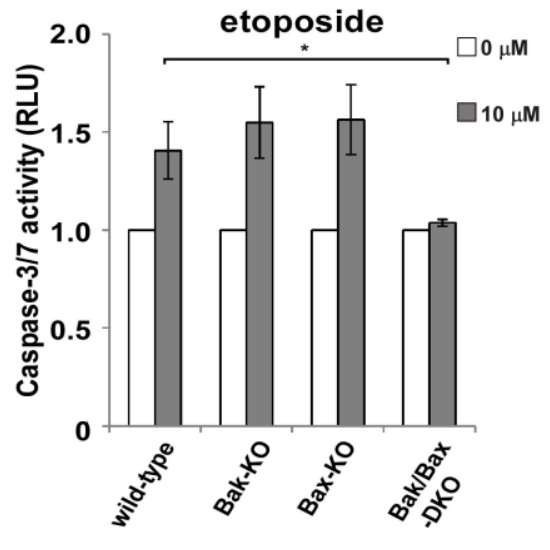

D

HCT116-BB-DKO

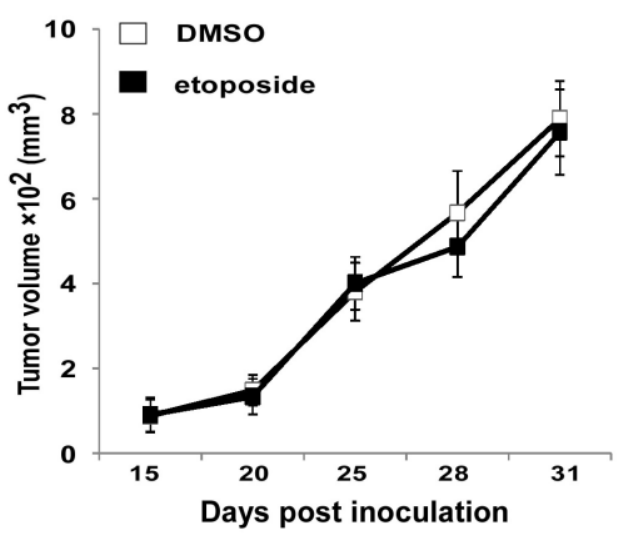


Figure 3.10. Etoposide induces tumor cell death and inhibits xenografted tumor growth dependent of Bak and Bax.

(A) Etoposide induced significant cell death in WT, Bak-KO and Bax-KO HCT116 cells but not in their Bak/Bax-DKO counterparts after 48 hours of treatment. (B) Caspase-3/7 activities were examined 24 hours after etoposide exposure. All the data are presented as mean \pm standard deviation of three different experiments. Asterisk indicates $\mathrm{P}<0.05\left({ }^{*}\right)$ or $\left.\mathrm{P}<0.01{ }^{* *}\right)$ by student's unpaired t test. (C-D) Growth of HCT116-WT tumors (C) and HCT116-Bak/Bax-DKO tumors (D) in athymic nude mice treated with the vehicle control or etoposide. Data are mean \pm standard deviation of tumor volumes of five animals in each group. 


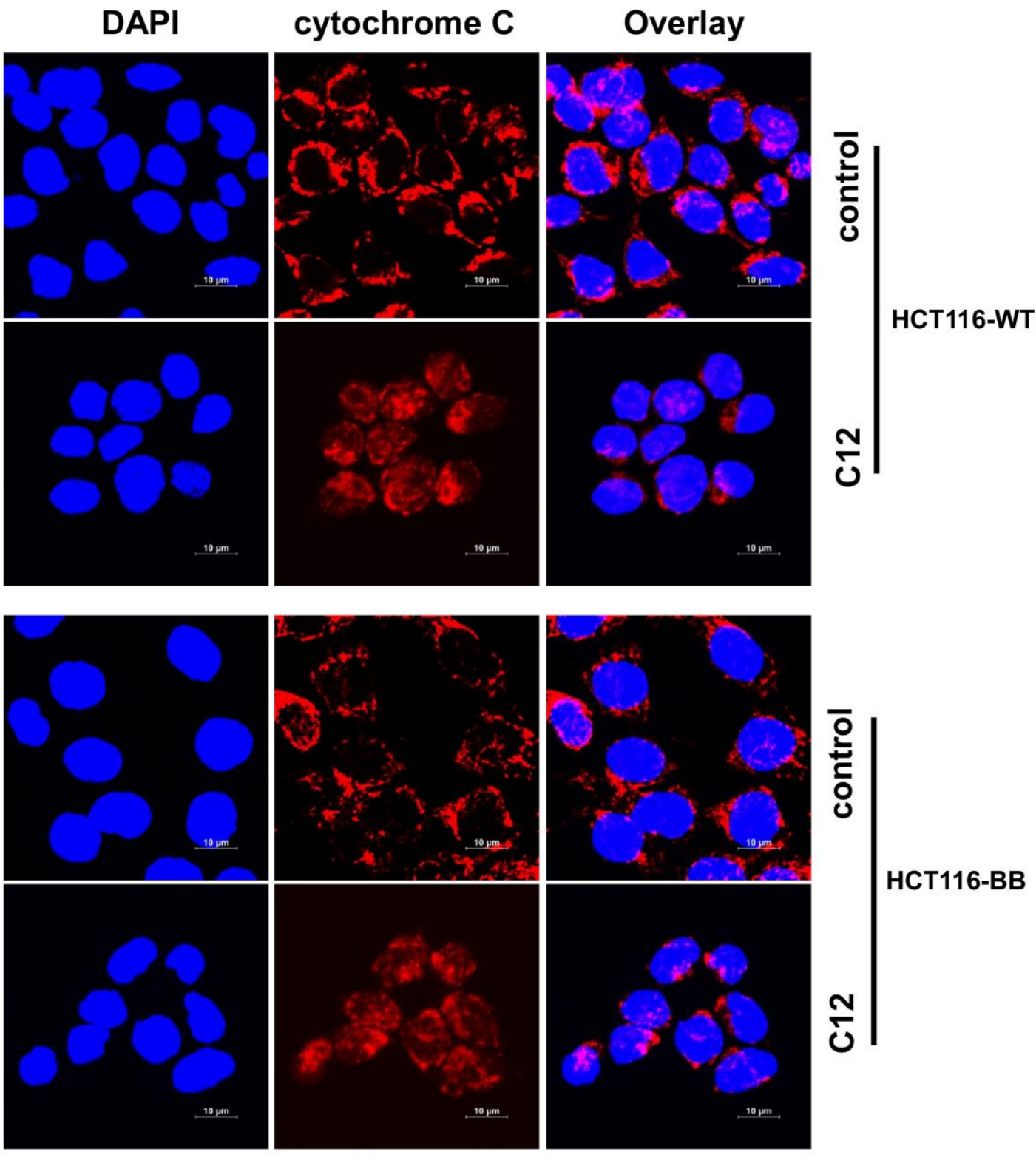


Figure 3.11. C12 triggered cytochrome $\mathrm{c}$ release in tumor cells independent of Bak and Bax.

(A-B) Cytochrome $\mathrm{c}$ was released from mitochondria in both HCT116-WT (A) and HCT116-Bax/Bak-DKO (B) cells upon treating with $100 \mu \mathrm{M}$ C12 of 3 hours. Typical confocal images are shown. Cytochrome $\mathrm{c}$ is shown in red, and DAPI is shown in blue. Scale bar, $10 \mu \mathrm{m}$. 
3.3.7. The inhibitory effects of $\mathrm{C} 12$ on tumor growth are independent of Bak and Bax.

To further investigate whether the Bax/Bak-independent effect of $\mathrm{C} 12$ to trigger apoptosis in vitro also occurred in vivo, WT and Bax/Bak-DKO HCT116 cells were inoculated into athymic nude mice. C12 reduced the growth of established HCT116-WT tumors and HCT116-Bak/Bax-DKO tumors to a similar degree (Figures 3.12A-B), indicating that Bak and Bax are not involved in anti-tumor activity of $\mathrm{C} 12$. Moreover, the level of activated caspase-3 and the percentage of TUNEL-positive cells were higher in C12-treated tumors than vehicle-treated tumors regardless of Bak and Bax expression levels (Figures 3.12C-F), suggesting that $\mathrm{C} 12$ inhibits tumor growth probably through inducing Bak/Bax-independent apoptosis. 
A

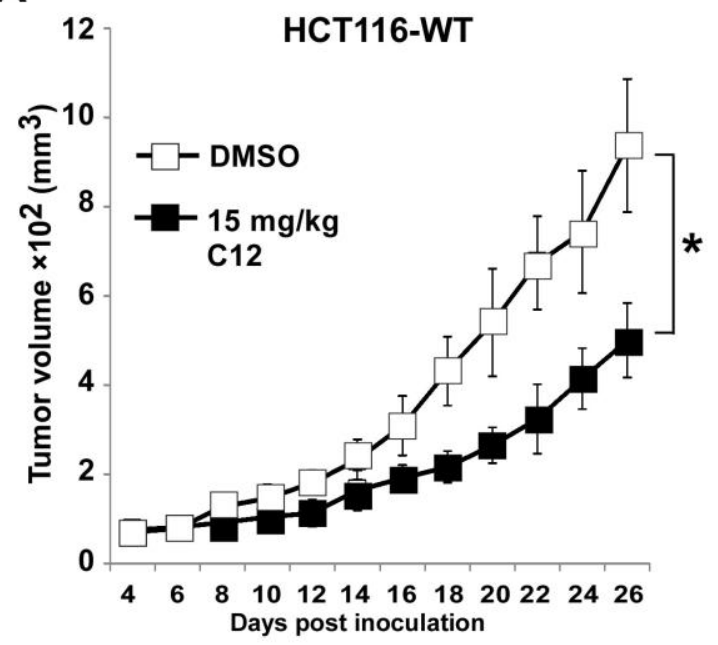

C
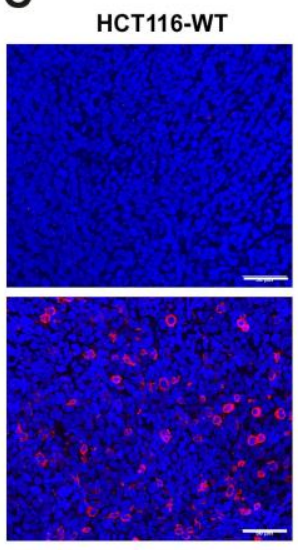

activated caspase 3

E
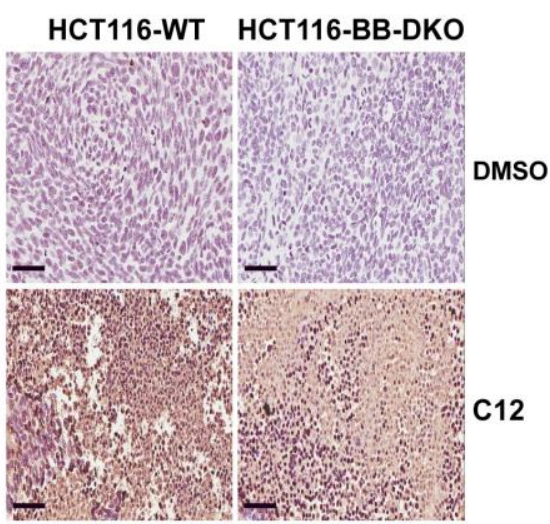

B

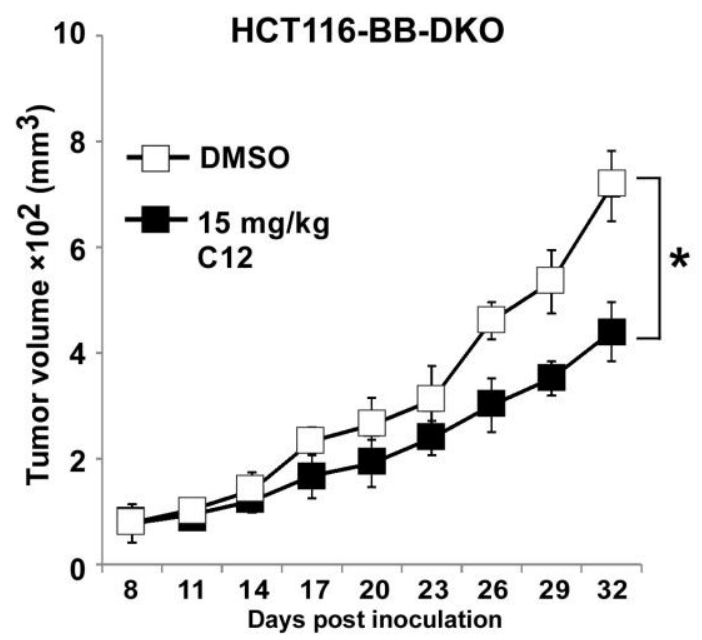

D

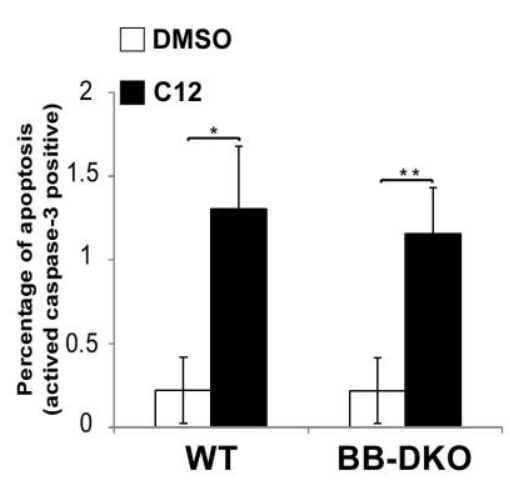

F

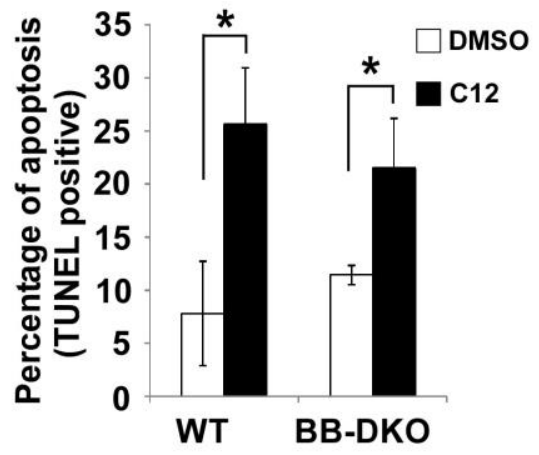


Figure 3.12. C12 inhibits xenografted tumor growth independent of Bak and Bax. (A-B) Growth of HCT116-WT tumors (A) and HCT116-Bak/Bax-DKO tumors (B) in athymic nude mice treated with vehicle control or C12 (15 mg/kg/day). Data are mean \pm standard deviation of tumor volumes of 8 animals in each group. (C) Apoptotic cells in tumor sections were detected by immunofluorescence staining of activated caspase-3. Representative images of tumor sections from DMSO- and C12-treated mice are shown. Scale bar, $50 \mu \mathrm{m}$. (D) The percentage of activated caspase-3 shown in (C) were quantified. Data are mean \pm standard deviation of three independent tumor sections. Asterisk indicates $\mathrm{P}<0.05\left({ }^{*}\right)$ or $\mathrm{P}<0.01\left(^{* \star}\right)$ by student's unpaired t test (E) Typical TUNEL labeling images of tumor sections from vehicle control- and C12-treated mice are shown. Scale bar, $60 \mu \mathrm{m}$. (F) The percentage of TUNEL-positive cells in tumors of C12-treated mice is higher than that of control mice. All data are mean \pm standard deviation of three independent tumor sections. Asterisk indicates $\mathrm{P}<0.05\left({ }^{*}\right)$ or $\mathrm{P}<0.01\left(^{* *}\right)$ by student's unpaired $t$ test. 
3.3.8. BH-3 only proteins Noxa and Puma are not involved in C12-induced tumor cell apoptosis.

BH3-only proteins are another class pro-apoptotic $\mathrm{Bcl}-2$ proteins, which share sequence homology only in the BH3 domain, including Noxa and Puma (149). To further elucidate whether $\mathrm{BH} 3-$ only proteins are involved in $\mathrm{C} 12$ cytotoxicity in tumor cells, we investigated two HCT116 cell lines in which Noxa expression was stably reduced by shRNA (Figure 3.13A) or Puma expression was eliminated genetically (Figure 3.13E) $(150,151)$. C12 caused similar levels of cell death and caspase-3/7 activation in HCT116 cell lines with reduced expression of Noxa or Puma compared with their corresponding counterparts expressing normal levels of Noxa or Puma (Figures 3.13B, 3.13D and 3.13F-G). In contrast, less cisplatininduced cytotoxicity was observed in HCT116 cells with decreased Noxa or Puma expression (Figures 3.13C and 3.13F-G). Overall, our results suggest that BH3only proteins are not critical for C12-induced apoptotic signaling. 
A

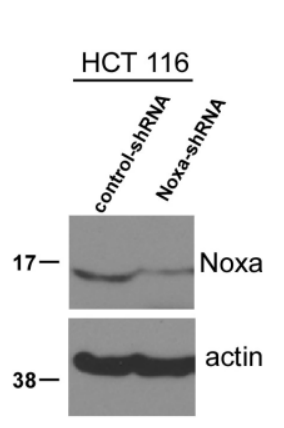

B

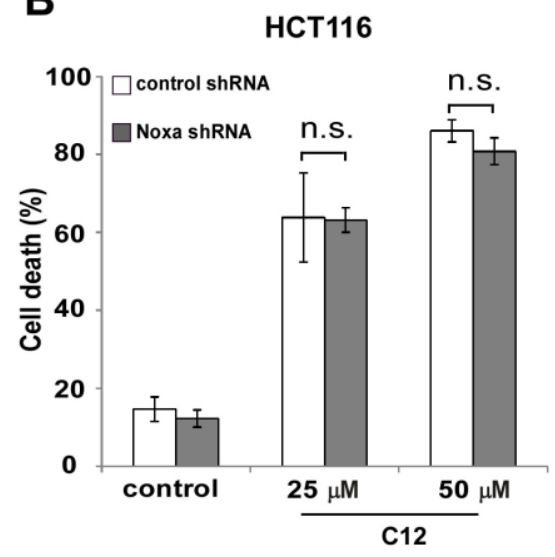

C

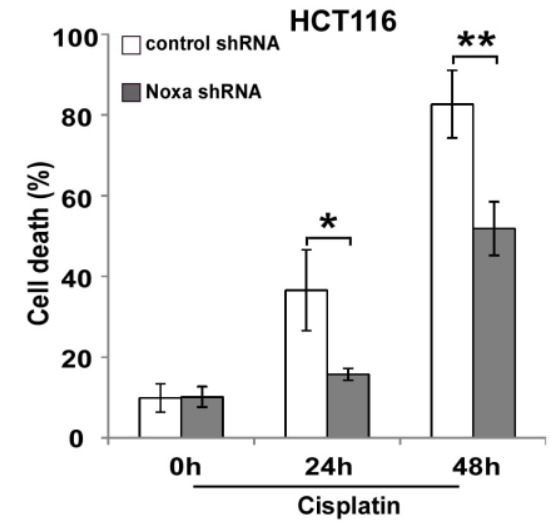

D

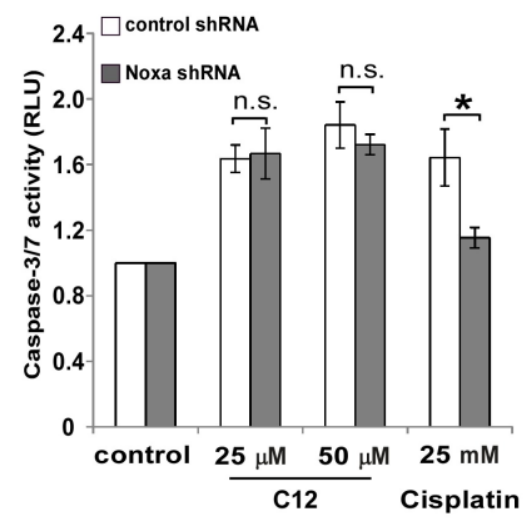

F

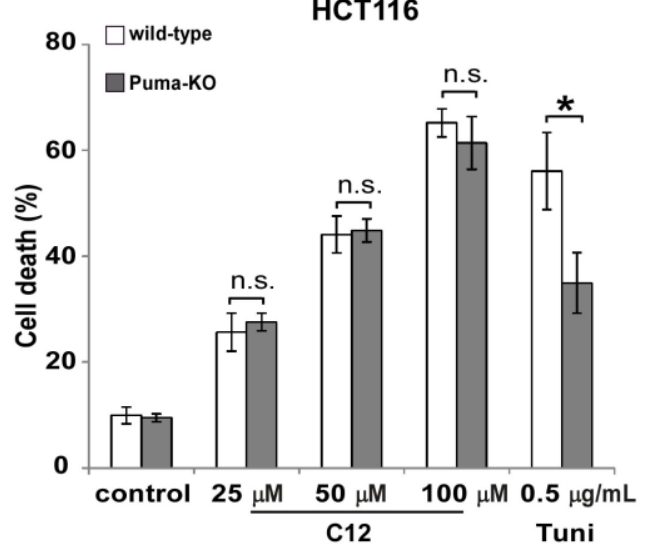

E

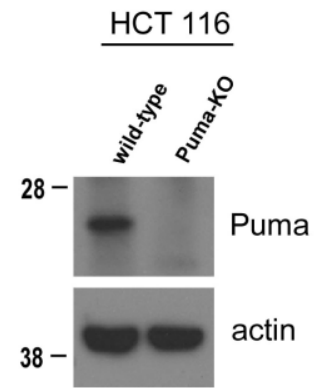

G

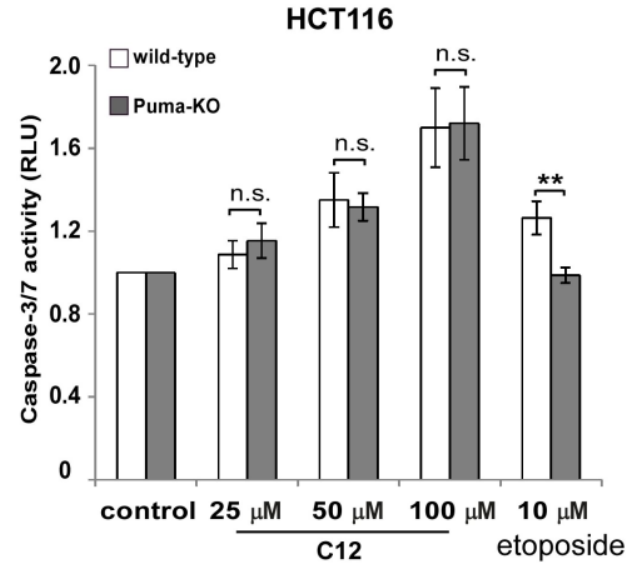


Figure 3.13. $\mathrm{C} 12$-induced tumor cell apoptosis is independent of $\mathrm{BH}-3$ only proteins Noxa and Puma.

(A) Noxa expression was stably reduced in HCT116 cells by shRNA. (B)

Following 24 hours treatment of $\mathrm{C} 12$, similar levels of cell death were detected in HCT116 cells expressing Noxa shRNA and their control counterparts. (C) Less apoptosis was detected in HCT116 cells with reduced Noxa expression upon treatment of cisplatin (50 mM). (D) Caspase-3/7activities were measured after 24 hour-incubation of $\mathrm{C} 12$ or cisplatin in the indicated HCT116 cells. (E) Puma expression in the indicated HCT116 cells was examined by western blot. (F) Cell death was measured after 24 hour-incubation of $\mathrm{C} 12$ or tunicamycin in Pumadeficient HCT116 cells or wild-type cells. (G) Caspase-3/7 activities were examined 24 hours after $\mathrm{C} 12$ or etoposide exposure. All data are presented as mean \pm standard deviation of three different experiments. Asterisk indicates $\mathrm{P}<$ $0.05\left(^{*}\right)$ or $\left.\mathrm{P}<0.01{ }^{* *}\right)$ by student's unpaired t test. ns, no significant. 


\section{DISCUSSION}

Despite growing evidence that the bacterial quorum-sensing molecule $\mathrm{C} 12$ induces apoptosis in various types of human tumor cells $(66,69-71)$, the relevance of C12 cytotoxicity to tumor growth in animals was unknown. Importantly, knowledge about the mechanism of C12-evoked tumor cell apoptosis has been limited. Here, we present the evidence that $\mathrm{C} 12$ preferentially triggers transformed cell apoptosis in vitro and inhibits transplanted tumor growth in vivo as a single agent independent of both anti- and proapoptotic Bcl-2 proteins. Furthermore, selective cytotoxicity of $\mathrm{C} 12$ on lung tumor cells and its inhibitory effects on tumor growth are likely related to upregulated PON2 expression in the tumor cells.

Many neoplastic cells show an increased ratio of anti-apoptotic to pro-apoptotic Bcl-2 proteins, which enables them to survive even under the conditions that would normally initiate apoptotic signaling $(121,152)$. An emerging strategy for cancer therapy is to overcome the resistance to apoptosis caused by aberrant Bcl-2 signaling in tumor cells (153). Recently, several small molecules triggering apoptosis independent of either pro- or anti-apoptotic $\mathrm{Bcl}-2$ proteins have been identified as potential anti-tumor drugs. Among them, the pentacyclic triterpenoid betulinic acid induces Bax/Bak-independent MOMP and subsequent apoptosis (154). Unlike C12, cytotoxic effects of betulinic acid are influenced by Bcl-2 overexpression and it is ineffective against epithelial tumors. Similarly, Bax/Bak is also nonessential in apoptotic signaling induced by chelerythrine (155), mitochondrial Kv1.3 channels inhibitors (156) or titanium dioxide $\left(\mathrm{TiO}_{2}\right)$ (157). 
The polyphenolic compound gossypol evokes Bax/Bak-independent apoptosis and inhibits Bcl-2-overexpressing tumor growth (158). Furthermore, Bcl-2 expression fails to influence human tumor cell apoptosis induced by the antibiotic agent Tetrocarcin-A or protein complex HAMLET $(128,129)$. Compared with those molecules, $\mathrm{C} 12$ is the first small molecule compound, to the best of our knowledge, inducing human tumor cell apoptosis in vitro as well as blocking tumor growth in vivo independent of both pro- and anti-apoptotic Bcl-2 proteins.

The apoptosis cascade induced by $\mathrm{C} 12$ in tumor cells is unique, evident by its rapid pro-apoptotic effects, such as depolarizing mitochondrial membrane potential within minutes (Figures 3.4 and 3.9), releasing cytochrome $\mathrm{c}$ into the cytosol within three hours (Figures 3.7 and 3.11 ) and maximally activating caspases within four hours (Figure 3.1). These distinctive pro-apoptotic features of C12 have not been observed in any other apoptosis paradigms of cancer cells, which might be attributed to the ability of $\mathrm{C} 12$ or its derivatives generated in tumor cells to directly permeabilize mitochondria (within minutes) without the involvement of pro- and anti-apoptotic Bcl-2 proteins.

As Pseudomonas aeruginosa produces $\mathrm{C} 12$ to control virulence factors, $\mathrm{C} 12$ alone could potentially promote infections, particularly in immune compromised patients (159). Published information about the safety of C12 in vivo is limited except that $\mathrm{C} 12$ exerts acute influence in immune responses in mice. For instance, $\mathrm{C} 12$ reduces innate immune responses acutely via disrupting TLR4- 
dependent NF-kB signaling in mice (62). In a murine model of dermal inflammation, intradermal injection of C12-loaded micelles leads to local immune cell infiltration within 24 hours (63). In our studies, administration of C12 for extended periods of time does not appear to cause significant toxicity to either normal or immunodeficient athymic nude mice (Figure 3.2), suggesting that longterm side effects of administrating $\mathrm{C} 12$ on animals might be limited.

Overall, our study reveals that $\mathrm{C} 12$ inhibits tumor growth in animals as a single agent, through inducing a unique $\mathrm{Bcl}-2$ protein-independent apoptotic cascade in tumors. Therefore, $\mathrm{C} 12$ is an ideal candidate of a lead compound for novel therapeutic agents for cancer. 
CHAPTER IV

PARAOXONASE 2 IS ESSENTIAL FOR THE PROLIFERATION OF NON-SMALL CELL LUNG CANCER CELL

\subsection{INTRODUCTION}

Human cancer originates from abnormal accumulation of a clonal population of somatic cells, which disrupts tissue homeostasis $(121,160)$. The initiation and progression of cancer involves genetic changes resulting in alterations in tissue development and homeostatic maintenance. Understanding how genetic alterations regulate tumor cell proliferation is essential for successful prevention, diagnosis and treatment of tumors.

Among the genes whose expression is altered in tumor cells is an enzyme named paraoxonase 2 (PON2) $(78,79,161-164)$. PON2 belongs to a highly conserved gene family (PON1, 2, 3) with lactonase and arylesterase activities (165-167). The three PON (1-3) genes are located in a cluster on human

chromosome 7 or mouse chromosome 6 , and PON proteins share about $66 \%$ of identity in amino acid sequences $(168,169)$. PON1 is secreted out of cells and associated with high-density lipid protein (HDL) in the blood following secretion (170). Like PON1, PON3 is also bound to HDL in the blood stream (171). In contrast, PON2 is an intracellular enzyme (172). PON1 and PON3 are primarily found in the liver, whereas PON2 is ubiquitously expressed in a number of 
tissues, including the liver, kidney, lung, heart, stomach, spleen, pancreas and macrophages (173). The three PON proteins possess overlapping but distinct enzymatic specificity $(76,174,175)$. Among various substrates of PON proteins, $\mathrm{N}$-acyl-homoserine lactones are of special interest, since they are quorumsensing molecules for communication among pathogenic bacteria $(59,84,176)$. Growing evidence demonstrate that PON proteins, particularly PON2, modulate many biological effects of $\mathrm{N}$-acyl-homoserine lactones, such as triggering stress signaling and apoptosis in host cells $(74,95,177,178)$. The hydrolysis of $\mathrm{N}$-acylhomoserine lactones by PON2 is expected to reduce quorum sensing by the bacteria and subsequently alleviate responses of host cells to $\mathrm{N}$-acyl-homoserine lactones.

In addition to its lactonase-esterase activity, PON2 also displays antioxidant activity (72). The antioxidant activity may contribute to the ability of PON2 to possess both anti-inflammatory and anti-apoptotic functions $(72,175)$ as well as explain why PON2 is often upregulated in cancer $(78,79,161-164)$. Normally PON2 mutations that reduce its lactonase activity do not alter its antioxidant activities, demonstrating that these two functions are mediated by different aspects of PON2. However, the role of PON2 lactonase activity in mediating apoptosis appears to be determined by the particular apoptosis signaling pathways involved $(138,179)$. For instance, PON2 mutants lacking lactonase activity seems to be more effective in preventing apoptosis in the Bax/Bakdependent canonical apoptotic signaling pathway than wild type PON2 (179). In 
contrast, PON2 uses its lactonase activity to promote a unique Bax/Bakindependent apoptosis in response to a homoserine lactone (138).

\subsection{MATERIALS and METHODS}

\subsubsection{Reagents}

$\mathrm{N}$-(3-oxododecanoyl)-homoserine lactone (C12) and polybrene were acquired from Sigma (St. Louis, MO). Propidium iodide (PI) was purchased from Invitrogen (Carlsbad, CA). All reagents were dissolved in Dimethyl sulfoxide (DMSO). Lipofectamine $2000 \AA$ was obtained from Invitrogen. Antibodies (Ab) used for western blot analysis were anti- $\beta$-actin mAb (Sigma), anti-human PON2 (Abcam; Cambridge, MA), anti-mouse PON2 pAb (antibodies-online; Atlanta, GA), antiSDHA mAb (Cell signaling; Danvers, MA), peroxidase-conjugated goat anti-rabbit IgG (Thermo; Waltham, MA), peroxidase-conjugated goat anti-mouse IgG (Thermo), peroxidase-conjugated mouse anti-rabbit lgG (TrueBlot $\circledast$, Rockland; Limerick, PA), peroxidase-conjugated rat anti-mouse lgG (TrueBlot $\AA$, Rockland).

\subsubsection{DNA plasmids}

Murine PON2 cDNA was purchased from Origene (Rockville, MD) and subsequently cloned into the lentiviral expression vector PCDH-CMV-MCS-EF1copGFP (System Biosciences; Palo Alto, $\mathrm{CA}$ ) to generate the plasmid pCDHCMV-mPON2-EF1-copGFP with the green fluorescent protein (GFP) functioning as an indicator of infection. The identity of the plasmids was validated by 
sequencing. Lentiviral shRNA plasmid for human PON2 and its counterpart with scramble sequence were purchased from Santa Cruz (Dallas, TX).

\subsubsection{Retrovirus and lentivirus production}

For lentivirus production, the package cell line human embryonic kidney (HEK)293T was transfected with the plasmid pCDH-CMV-mPON2-EF1-copGFP, human PON2 shRNA plasmid or their corresponding control plasmids along with the helper plasmids pMDLg/pRRE, pRSV.Rev, and pMDG2.0 (Addgene; Cambridge, MA) using the Lipofectamine $2000 \AA$ transfection reagent. Medium containing lentivirus was collected 48-72 hours following transfection.

\subsubsection{Generation of cell lines and cell culture}

Non small cell lung carcinoma cell lines A549 and $\mathrm{NCl}-\mathrm{H} 1299$ were purchased from ATCC (Bethesda, MD). HEK293T cells were also obtained from ATCC. Human bronchial epithelial (HBE) cells immortalized by expressing hTERT and Cdk4 were acquired from Professor Geoffrey Clark (University of Louisville). To generate cells with reduced PON2 expression or empty vector control, A549, $\mathrm{NCl}-\mathrm{H} 1299, \mathrm{HEK} 293 \mathrm{~T}$ and HBE cells were infected with the medium containing corresponding lentivirus and $10 \mu \mathrm{g} / \mathrm{ml}$ of polybrene to enhance infection efficiency. Stable cell lines were generated by culturing cells in the medium containing $2 \mu \mathrm{g} / \mathrm{ml}$ puromycin. A549 and $\mathrm{NCl}-\mathrm{H} 1299$ cells expressing murine PON2 were generated by infection with the lentiviral supernatants containing 10 $\mu \mathrm{g} / \mathrm{ml}$ of polybrene. Almost all of the infected cells (>95\%) were GFP positive as 
evaluated by flow cytometry (FACScalibur, Beckon Dickinson; San Jose, CA). All of cell lines were cultured in Dulbecco's Modified Eagle Medium (DMEM) medium (Mediatech; Manassas, VA) supplemented with $10 \%$ fetal bovine serum (FBS) (Gemini; West Sacramento, CA), $100 \mathrm{U} / \mathrm{ml}$ penicillin (Mediatech), and100 $\mu \mathrm{g} / \mathrm{ml}$ streptomycin (Mediatech) in an incubator with $95 \%$ humidity and $5 \% \mathrm{CO}_{2}$ at $37^{\circ} \mathrm{C}$.

\subsubsection{Cell viability assay}

The indicated cells were cultured in 48 -well tissue culture plates with $1 \times 10^{4}$ cells in each well 24 hours before treatment with apoptotic stimuli. At the indicated time points following the treatment, cells were trypsinized and resuspended in growth medium containing $1 \mu \mathrm{g} / \mathrm{ml}$ propidium iodide. Cell viability was evaluated by a PI exclusion approach using flow cytometry (FACScalibur). The experiments were performed in triplicates. The percentage of cell death is calculated as 100 minus the percentage of viable cells. .

4.2.6. Cell cycle analysis and cell proliferation assay

For cell cycle analysis, $5 \times 10^{5}$ cells were sedimented ( $300 \times \mathrm{g}$ for 5 minutes) and washed twice with $500 \mu \mathrm{l} 1 \times$ PBS. Cells were then fixed with $1 \mathrm{ml} 70 \%$ ethanol in $1 \times \mathrm{PBS}$ at $4^{\circ} \mathrm{C}$ overnight. After centrifugation ( $300 \times \mathrm{g}$ for 5 minutes), cells were washed twice with 1 x PBS and resuspended in $500 \mu \mathrm{l} 1$ x PBS. $50 \mathrm{U}$ RNase A (Qiagen; Valencia, CA) were added to samples followed by incubation at $37^{\circ} \mathrm{C}$ for 1 hour. Then propidium iodide $(5 \mu \mathrm{g})$ was added to samples which 
were incubated for 30 minutes at $37^{\circ} \mathrm{C}$ before flow cytometric analysis (FACScalibur). To evaluate cell proliferation, $1.5 \times 10^{4}$ cells were plated in wells of a 12-well tissue culture plate and the number of the cells in each well was determined using a hemocytometer.

\subsubsection{Western blot analysis}

Cell pellets were collected, and whole cell lysates were generated by resuspending cells in RIPA lysis buffer (150 mM sodium chloride, $1 \%(\mathrm{v} / \mathrm{v})$ Triton $\mathrm{X}-100,0.5 \%$ sodium deoxycholate, $0.1 \%$ sodium dodecyl sulphate, and $50 \mathrm{mM}$ Tris, pH 8.0) containing protease inhibitors (Complete, Roche; Indianapolis, IN). To remove cell debris and nuclear materials, cells were first sonicated for 30 seconds at 10\% amplitude (Sonic Dismembrator, Model 500, Fischer Scientific; Hampton, $\mathrm{NH}$ ) before centrifugation at $13,000 \times \mathrm{rpm}$ at $4^{\circ} \mathrm{C}$ for 10 minutes, and the supernatant was collected. The protein concentration was determined using the BCA Assay (Pierce; Rockford, IL). Thirty micrograms of protein sample were loaded on a $4-12 \%$ Bis-Tris gel (Bio-Rad; Hercules, CA) and transferred to PVDF (Millipore; Billerica, MA). The membrane filters were incubated in buffer containing $1 \times$ PBS, $0.2 \%(\mathrm{v} / \mathrm{v})$ Tween 20 , and $10 \%(\mathrm{w} / \mathrm{v})$ nonfat dry milk (BioRad) with appropriate primary antibodies at $4^{\circ} \mathrm{C}$ overnight or at $25^{\circ} \mathrm{C}$ for about three hours. After three 10 minute wash with $1 \times$ PBS/0.2\% Tween solution, the membrane filters were then incubated with the appropriate peroxidase-coupled secondary antibodies at $4{ }^{\circ} \mathrm{C}$ overnight or for at $25^{\circ} \mathrm{C}$ for 
three hours. Proteins were detected using the enhanced chemiluminescent detection system (Thermo).

\subsubsection{Lung tumor tissues from patients}

Tumor tissues along with corresponding adjacent normal tissues from non small cell lung carcinoma (NSCLC) patients were acquired from the James Graham Brown Cancer Center Bio-Repository at University of Louisville following an approved IRB protocol. Frozen tissue samples were resuspended in tissue protein extraction reagent (Thermo Fisher) supplemented with protease inhibitors (Complete, Roche; Indianapolis, IN) and phosphatase inhibitors (PhosSTOP, Roche). Following homogenization and centrifugation, protein concentration was determined by BCA assay (Thermo Fisher). Thirty micrograms of total protein were electrophoresed in 4-12\% Bis/Tris gels (Bio-Rad; Hercules, CA). PON2 was detected by western blot.

\subsubsection{Immunoprecipitation}

A549 cells $\left(1 \times 10^{8}\right)$ were collected and washed once with $1 \times$ PBS. Cells were resuspended in cell lysis buffer containing $12 \mathrm{mM}$ HEPES (pH 7.5), 1.7 mM Tris$\mathrm{HCl}\left(\mathrm{pH}\right.$ 7.5), $100 \mathrm{mM} \mathrm{KCl}, 140 \mathrm{mM}$ mannitol, $23 \mathrm{mM}$ sucrose, $2 \mathrm{mM} \mathrm{KH}_{2} \mathrm{PO}_{4}, 1$ $\mathrm{mM} \mathrm{MgCl} 2,0.67 \mathrm{mM}$ EGTA, and 0.6 mM EDTA supplemented with protease inhibitors (Complete; Roche Diagnostics, Indianapolis, IN). The cells were broken open by a dounce homogenizer. The whole cell lysate was centrifuged at $300 \mathrm{xg}$ for 10 minutes at $40 \mathrm{C}$ to remove large cellular debris and nuclei. The 
remaining supernatant was centrifuged at $10,000 \times \mathrm{g}$ for 10 minutes at $4^{\circ} \mathrm{C}$, and the resulting mitochondria-enriched pellet was resuspended in mitochondrial lysis buffer containing $50 \mathrm{mM}$ Tris (pH7.5), $150 \mathrm{mM} \mathrm{NaCl}, 0.05 \%$ SDS, $1 \% \mathrm{NP}-40$ and protease inhibitors. The mitochondrial lysate was acquired by passing through a syringe with a needle (Gauge 30) 10 times to break open mitochondria.

Following centrifuged at $16,000 \times \mathrm{g}$ for 10 minutes at $4^{\circ} \mathrm{C}$, the protein concentration of the supernatant was determined by a BCA assay. Mitochondrial lysate (4 mg) was incubated with $20 \mu \mathrm{l}$ anti-Rabbit Ig IP agarose beads (50\% slurry, TrueBlot ${ }^{\circledR}$, Rockland; Limerick, PA) at $4^{\circ} \mathrm{C}$ for 30 minutes to preclear nonspecific protein interaction. Precleared mitochondrial lysate was split into two equal parts. One part was untouched and the other part was incubated with $5 \mu \mathrm{l}$ anti-PON2 antibodies (Abcam) overnight at $4^{\circ} \mathrm{C}$. Then anti-Rabbit Ig IP agarose beads ( $50 \mu \mathrm{l}$ of $50 \%$ bead slurry) were added to the mixture of protein lysate and antibodies and incubated with gentle rocking for 3 hours at $4^{\circ} \mathrm{C}$. Following centrifugation at $16,000 \times \mathrm{g}$ for 2 minutes at $40 \mathrm{C}$, the beads were washed 3 times with $500 \mu \mathrm{l}$ of mitochondrial lysis buffer. The beads with bound proteins were sent to the Genome Center at University of California, Davis for proteomic analysis.

\subsubsection{Proteome data analysis}

The resulting. msf files from Proteome Discoverer were loaded into Scaffold Q+S v4.3.2. Scaffold was used to calculate the false discovery rate using the peptide and protein prophet algorithms. The results were annotated with mouse gene 
ontology information from the Gene Ontology Annotations Database. Heat maps were compiled in the open-source statistical programming language R. Proteome output as Log2 fold change of control was analyzed with the heatmap.2 function of the gplots package, using the methods described by Key et al. Pearson's correlation coefficient was used to measure distance, and the Ward method (ward.D) was the agglomeration method for row and column clustering.

\subsubsection{Statistical analysis}

Statistical analysis was performed using the student t-test and the two way analysis of variance (ANOVA). A $P$ value $<0.05$ was considered significant. 


\subsection{RESULTS}

4.3.1. PON2 expression is enhanced in human lung tumor tissues and oncogenically transformed HBE cells.

It has been shown that PON2 upregulation in some cancer cells, including lung cancer cell lines, enables cancer cells to become resistant to conventional therapeutic drugs (79). To determine whether PON2 expression is enhanced in human lung cancer, we examined PON2 protein levels in tumor tissues of nonsmall cell lung carcinoma (NSCLC) patients by western blot. Among eleven samples from patients, we found that PON2 was overexpressed in eight of lung cancer tissues compared with corresponding adjacent normal tissues, whereas its expression was slightly decreased in three of them (Figure 4.1A). As Rastransformed HBE displayed higher levels of apoptosis compared with their untransformed counterparts upon C12 treatment (Figures 3.1A-B), PON2 expression was also increased in transformed HBE cells (Figure 4.1C). These observations provide more evidence that oncogenic transformation enhances PON2 expression. 
A

$\frac{1}{\mathrm{TN}} \frac{2}{\mathrm{~T} N} \frac{3}{\mathrm{TN}} \frac{4}{\mathrm{~T} N} \frac{5}{\mathrm{~T} N} \frac{6}{\mathrm{~T} N} \frac{7}{\mathrm{~T} N} \frac{8}{\mathrm{~T} N} \frac{9}{\mathrm{~T} N} \frac{10}{\mathrm{~T} N} \frac{11}{\mathrm{TN}}$

B

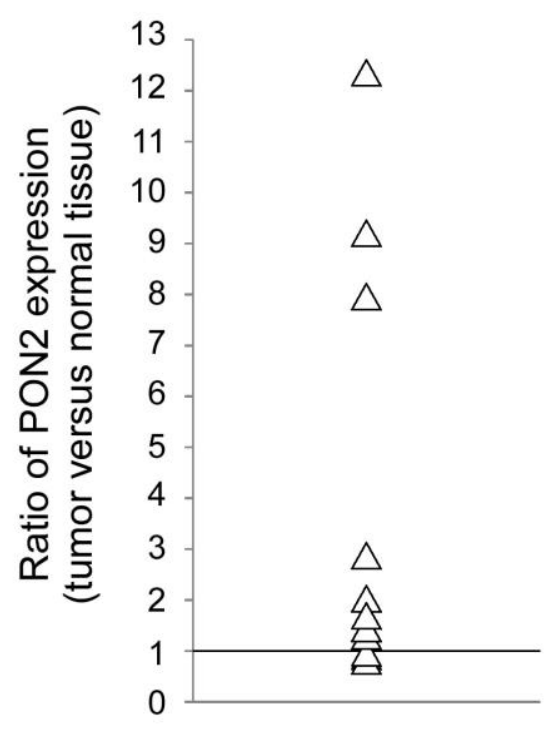

C

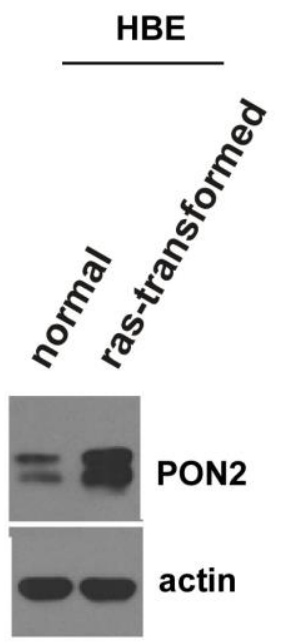


Figure 4.1. PON2 expression is enhanced in human lung tumor tissues and oncogenically transformed HBE cells. (A) Expression of PON2 in NSCLC tissue specimens and corresponding adjacent normal tissues from 11 patients were evaluated by western blot. Samples $1-4,6,8,9,11$ were from adenocarcinoma patients, whereas samples $5,7,10$ were from squamous cell carcinoma patients. T, tumor; N, normal. (B) The intensities of bands in (A) were quantified using ImageJ software $(\mathrm{NIH})$. To normalize loading variation, the relative levels of PON2 were calculated by dividing the PON2 value into the corresponding value for actin. The data were shown as a ratio of PON2 levels in a tumor tissue sample versus its corresponding normal tissue, and the value bigger than 1 indicates that PON2 expression is increased in tumor tissues. Differential expression of PON2 in tumor versus normal tissues is significant with the value of "P" smaller than 0.01 as calculated by student's paired t test. (C) The expression of PON2 and PON3 in primary HBE cells and their transformed counterparts was determined by western blot. 
4.3.2. PON2 blocks chemotherapeutic drug-triggered apoptosis in NSCLC cells.

To examine the role of PON2 in NSCLC cells' apoptotic responses, we investigated the prospective involvement of endogenous PON2 in cytotoxicity of chemotherapeutic drugs. We employed shRNA to stably reduce PON2 expression in human NSCLC cell lines A549 and NCl-H1299, and endogenous PON2 levels were markedly decreased (Figures 4.2A and 4.2D). Increased cell death and caspase-3/7 activation observed in PON2-deficient cells in response to the conventional apoptotic stimuli actinomycin D (Figures 4.2B-4.2E-F). This is in agreement with previous studies, and the anti-apoptotic effects of PON2 is thought to be associated with anti-oxidant activities of PON2 $(78,79)$. 
A

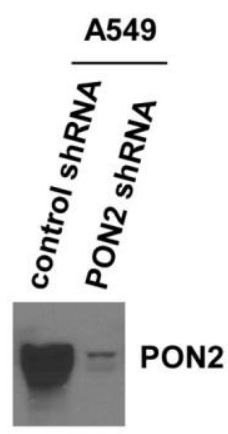

actin

D

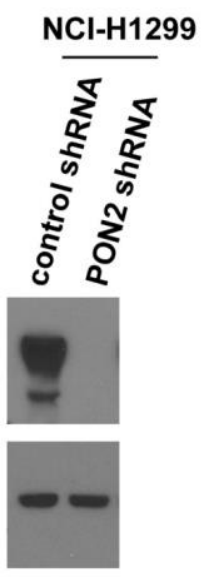

B

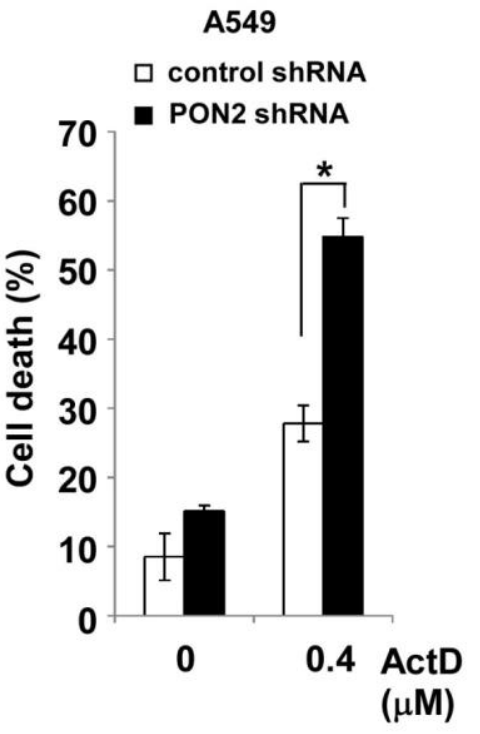

E

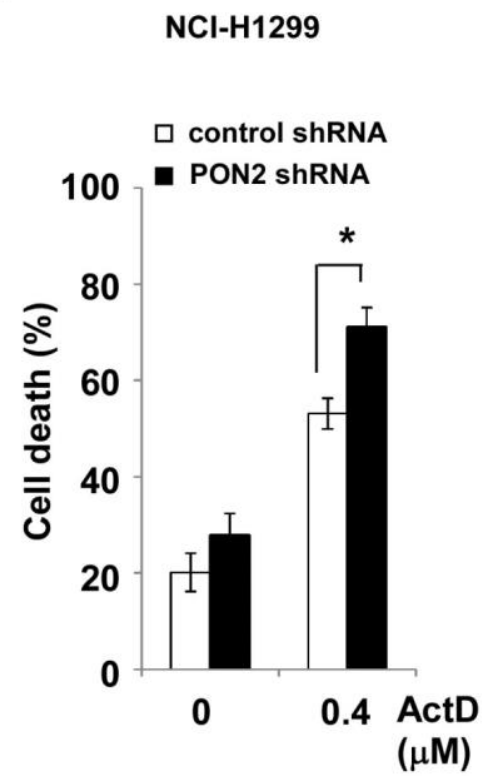

C

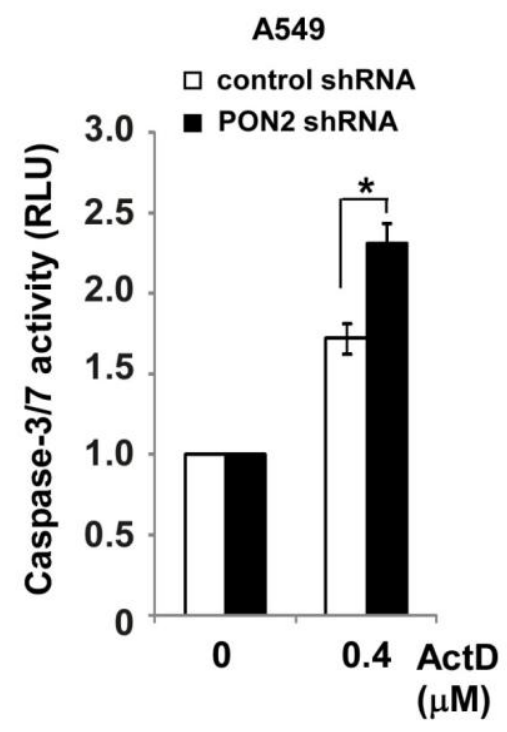

F

$\square$ control shRNA

- PON2 shRNA

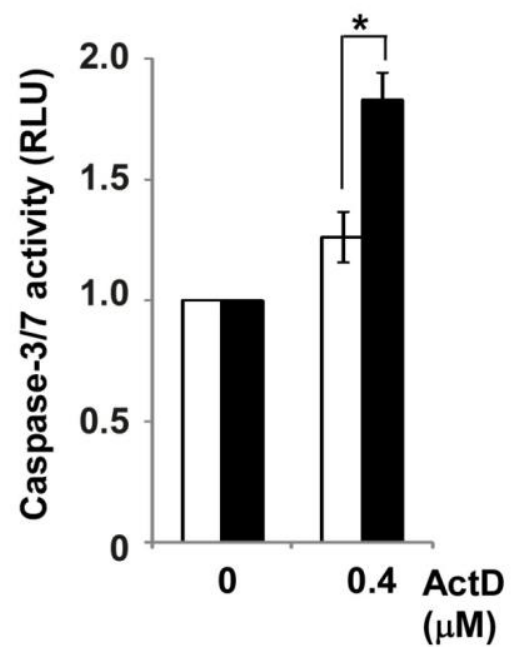


Figure 4.2. PON2 blocks chemotherapeutic drug-triggered apoptosis.

(A) PON2 expression in A549 cells was stably reduced by shRNA, and PON2 expression was determined by western blot. (B) The viability of A549 was measured 32 hours after ActD treatment. (C) ActD induced less cell death in A549 cells with reduced PON2 expression than control vector cells 48 hours after treatment. (D) Stable reduction of PON2 expression in $\mathrm{NCl}-\mathrm{H} 1299$ cells was evaluated by western blot. (E) $\mathrm{NCl}-\mathrm{H} 1299$ cell viability was assessed 24 hours following ActD exposure. (F) Less apoptosis was detected in $\mathrm{NCl}-\mathrm{H} 1299$ cells with reduced PON2 expression upon ActD treatment for 24 hours. "ActD"; actinomycin $\mathrm{D}$. All data shown are mean \pm standard deviation of three independent experiments. Asterisks indicate $P$ values of $<0.05\left(^{*}\right)$ by Student's unpaired t test. 
4.3.3. PON2 is required in $\mathrm{C} 12$ cytotoxicity in human NSCLC cells. Overexpression of PON2 promotes cytotoxicity of C12 in non-transformed MEF and HEK293T cells (138), but the role of endogenous PON2 in C12-induced apoptotic signaling is unclear. To further explore the mechanism of $\mathrm{C} 12$ triggered apoptosis, we investigated the prospective involvement of endogenous PON2 in C12 cytotoxicity in NSCLC cells. Treatment with C12 elicited less cell death and caspase-3/7 activation in A549 and $\mathrm{NCl}-\mathrm{H} 1299$ cells lacking PON2 expression (Figure 4.3). The distinct roles of PON2 in apoptosis of NSCLC cells induced by $\mathrm{C} 12$ and therapeutic drugs provide more evidence that a novel form of apoptosis is triggered by $\mathrm{C} 12$ (Figure 2.10). 
A

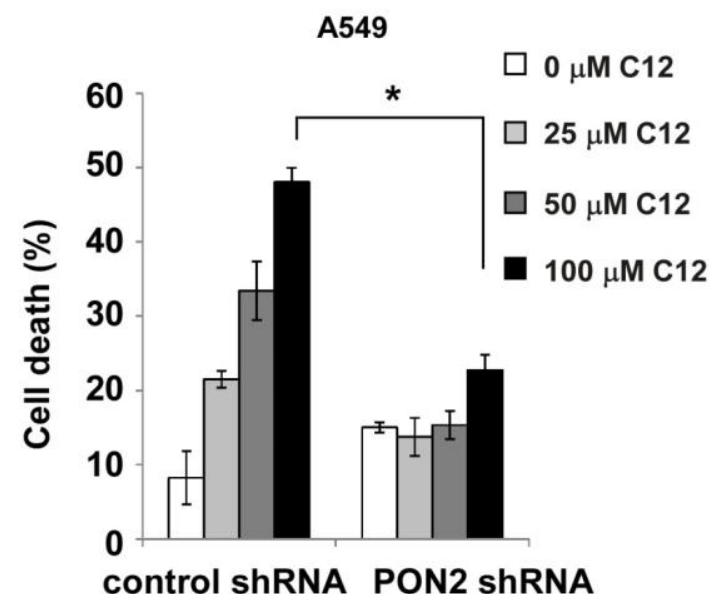

C

NCl-H1299

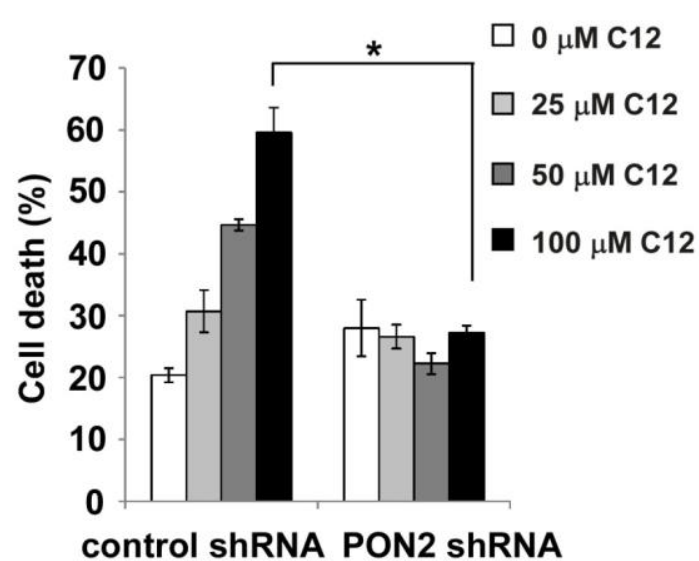

B

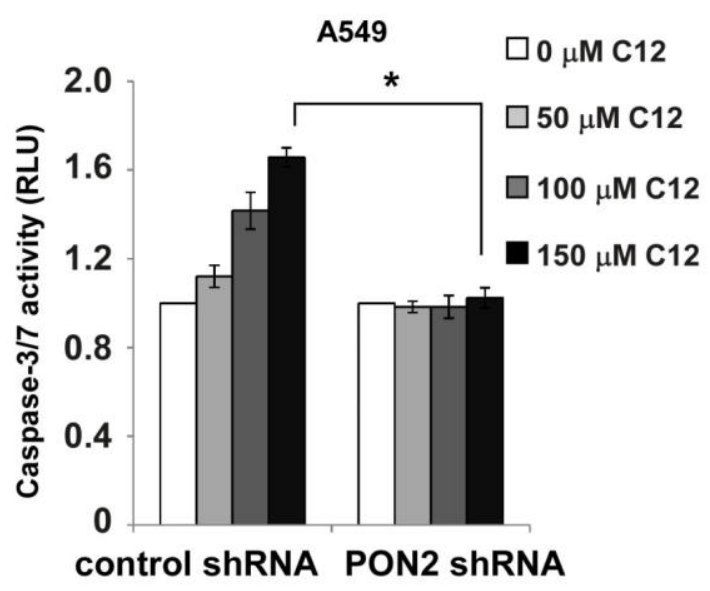

D

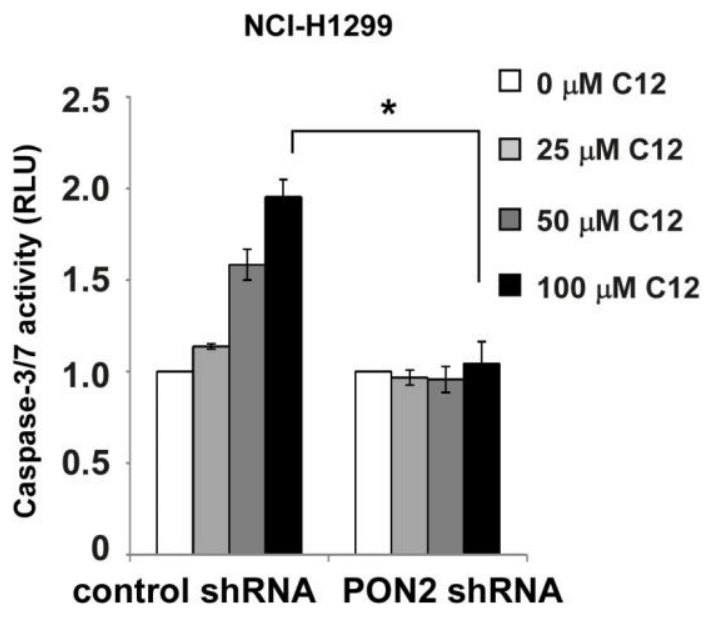


Figure 4.3. PON2 is required in C12's cytotoxicity on NSCLC cells (A) C12 induced less cell death in A549 cells with reduced PON2 expression than control vector cells 32 hours after treatment. (B) Upon treatment with different doses of $\mathrm{C} 12$ for 32 hours, less caspase-3/7 activation was detected in cells with reduced PON2 expression. (C) $\mathrm{C} 12$ induced less cell death in $\mathrm{NCl}-\mathrm{H} 1299$ cells with reduced PON2 following 24-hour treatment. (D) Less apoptosis was detected in $\mathrm{NCl}-\mathrm{H} 1299$ cells with reduced PON2 expression upon $\mathrm{C} 12$ treatment. All data shown are mean \pm standard deviation of three independent experiments. Asterisks indicate P values of $<0.05\left(^{*}\right)$ or $<0.01$ $\left.{ }^{* *}\right)$ by Student's unpaired $t$ test. 
4.3.4. Murine PON2 sensitizes human lung tumor cells with reduced endogenous PON2 expression to $\mathrm{C} 12$.

To validate the role of PON2 in mediating C12-induced apoptosis, we stably overexpressed murine PON2 cDNA in A549 cells deficient in PON2 expression by retroviral infection (Figure 4.4A). Upon treatment with C12, we observed more cell death and caspase-3/7 activation in PON2-deficient A549 cells overexpressing murine PON2 compared to vector control and parental cells (Figure 4.4B). Similarly, stable overexpression of murine PON3 in PON2knockdown $\mathrm{NCl}-\mathrm{H} 1299$ cells was confirmed by western blot (Figure 4.4C). We found that $\mathrm{C} 12$ induced more cell death and elicited higher caspase-3/7 activation in PON2-knockdown NCl-H1299 cells expressing mouse (Figure 4.4D). 
A Human PON2 shRNA

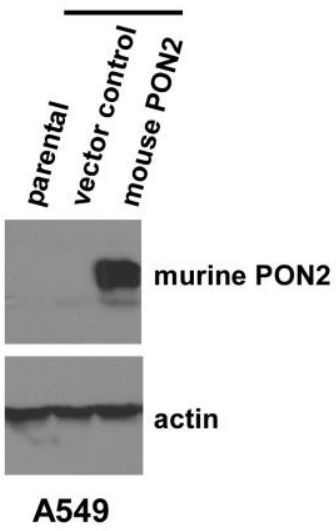

C Human PON2 shRNA

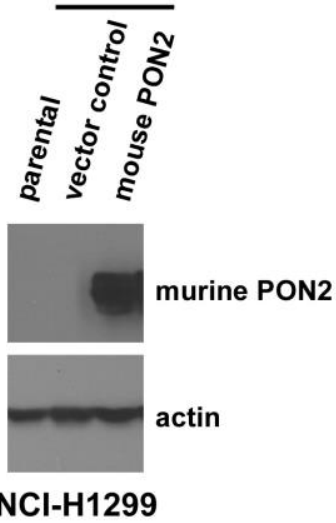

B

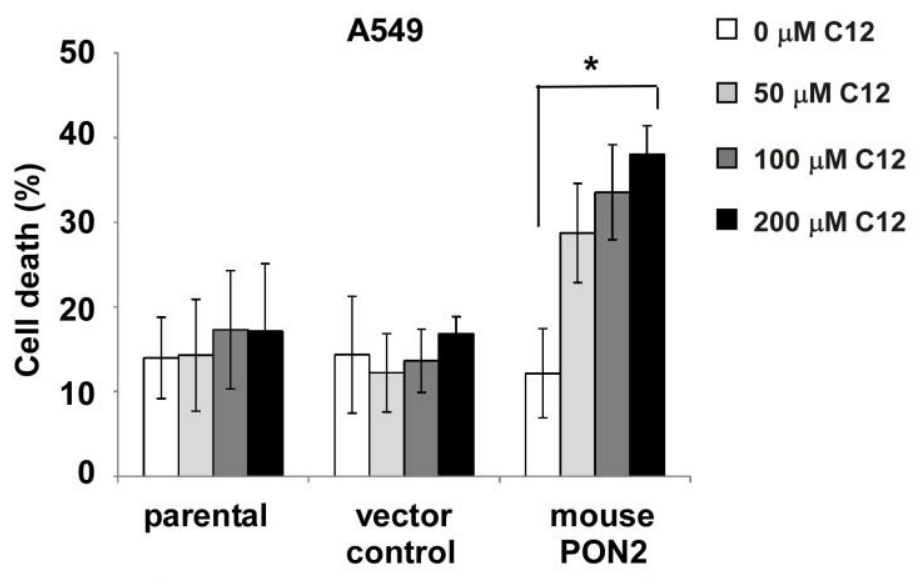

Human PON2 shRNA

D

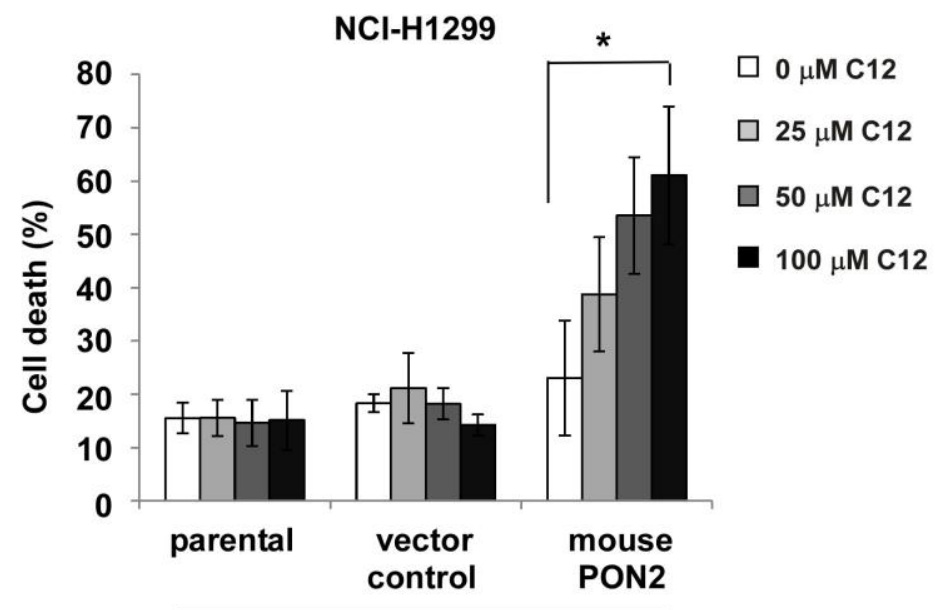

Human PON2 shRNA 
Figure 4.4. Murine PON2 sensitizes human lung tumor cells with reduced endogenous PON2 expression to C12. (A) Murine PON2 cDNA was stably overexpressed in A549 cells with reduced PON2 expression by retroviral infection. Expression levels of PON2 were determined by western blot. (B) After treating with different doses of $\mathrm{C} 12$ for 24 hours, more cell death were detected in PON2-knockdown A549 cells with increased mouse PON2 expression comparing to vector control and parental cells. (C) Stable overexpression of murine PON3 in PON2-knockdown NCl-H1299 cells was examined by western blot. (D) $\mathrm{C} 12$ induced more cell death in PON2-knockdown NCl-H1299 cells expressing mouse PON2 after 24 hours treatment. All data shown are mean \pm standard deviation of three independent experiments. Asterisks indicate $P$ values of $<$ $0.05\left(^{*}\right)$ by Student's unpaired $t$ test. 
4.3.5. PON2 is essential for C12-triggered cell death in HEK-293T and HBE cells.

To further explore the involvement of PON2 in apoptotic signaling in nontransformed cells, endogenous PON2 expression was stably reduced in Human Embryonic Kidney-293T (HEK-293T) cells, whose viability was assessed upon treatment with $\mathrm{C} 12$ (Figures 4.5A-B). Reducing PON2 expression in HEK-293 cells enabled cells resistant to C12. Furthermore, we stably decreased PON2 expression in immortalized human bronchial epithelial (HBE) cells (180) and measured cell viability upon C12 exposure (Figures 4.5C-D). Reducing PON2 expression in HBE cells de-sensitized cells to C12. Overall, these data provide evidence that PON2 plays a similar role in apoptotic signaling in both transformed and non-transformed cells. 
A

HEK-293T

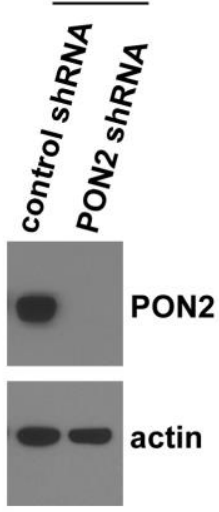

C

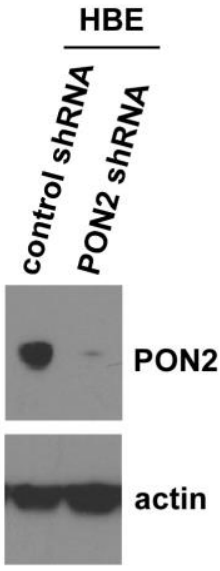

B

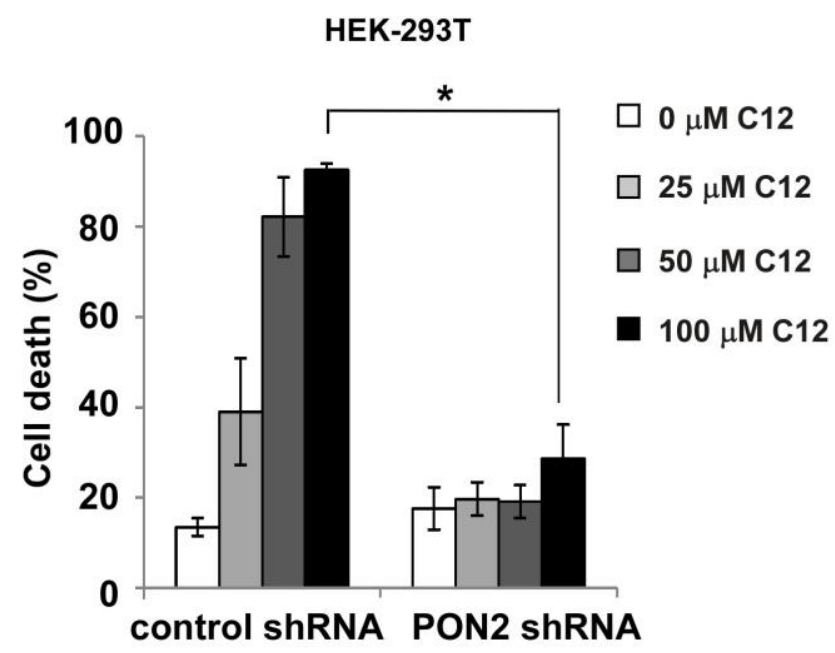

D

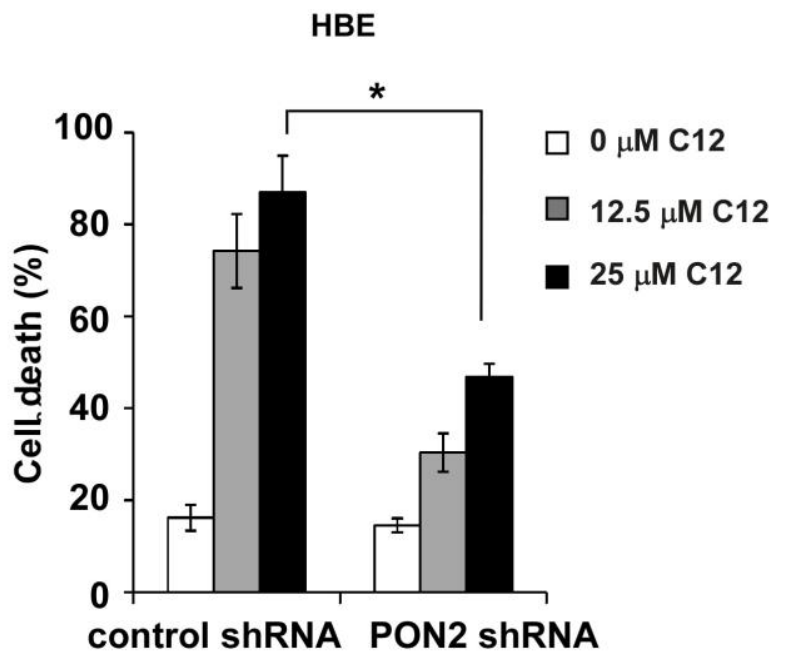


Figure 4.5. PON2 is essential for C12-triggered cell death in HEK-293T and HBE cells. (A) PON2 expression was stably reduced in HEK-293Tcells. (B) The viability of HEK-293Tcells was measured 24 hours after C12 treatment. (C) Stable decrease of PON2 expression in human bronchial epithelial (HBE) cells was determined by western blot. (D)The viability of HBE was evaluated 24 hours following $\mathrm{C} 12$ exposure. Mean \pm standard deviation for three independent experiments are shown. For all the data, ${ }^{*}, \mathrm{P}<0.05$, Student's unpaired $t$ test. 
4.3.6. PON2 is essential for human lung tumor cell proliferation but not nontransformed cell proliferation.

During the process of generating $\mathrm{A} 549$ and $\mathrm{NCl}-\mathrm{H} 1299$ cells lacking PON2 expression, we observed that those cells proliferated much slower than their vector control counterparts. To determine whether or not PON2 is essential for human lung tumor cell proliferation, we measured the proliferation of A549 cells and $\mathrm{NCl}-\mathrm{H} 1299$ expressing PON2 shRNA or the empty vector control. We found that proliferation of $\mathrm{NCl}-\mathrm{H} 1299$ and $\mathrm{A} 549$ cells with reduced PON2 expression was slower compared to that of their empty vector expressing counterparts (Figure 4.6A-B), implicating a role of PON2 in lung tumor cell proliferation. To determine whether or not PON2 is involved in the proliferation of non-transformed cells, we measured the proliferation of HEK-293T and HBE expressing PON2 shRNA or the empty vector control. It was found that HEK293T and HBE cells lacking PON2 expression grew at the same rate as their counterparts expressing the empty vector (Figure 4.6C-D), indicating that PON2 is not involved in non-transformed cell proliferation. Overall, these data indicate that PON2 mediates apoptosis independently of its function to modulate cell proliferation. 
A

A549

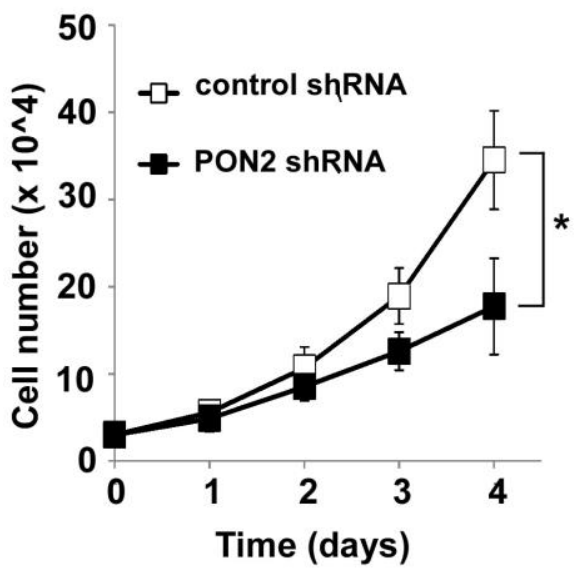

C

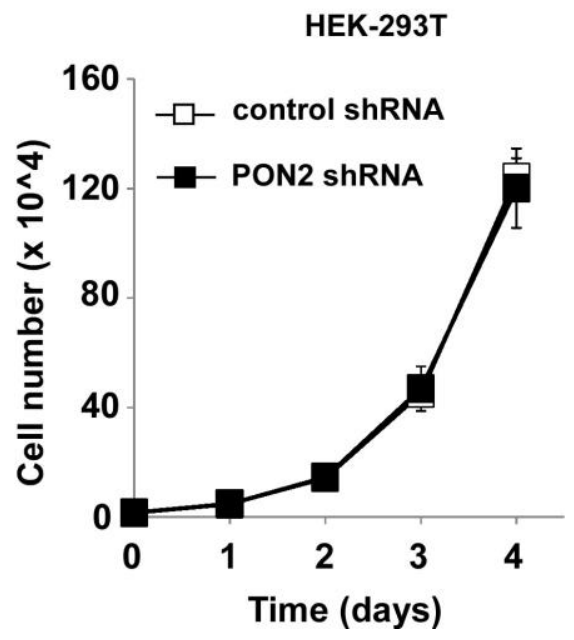

B

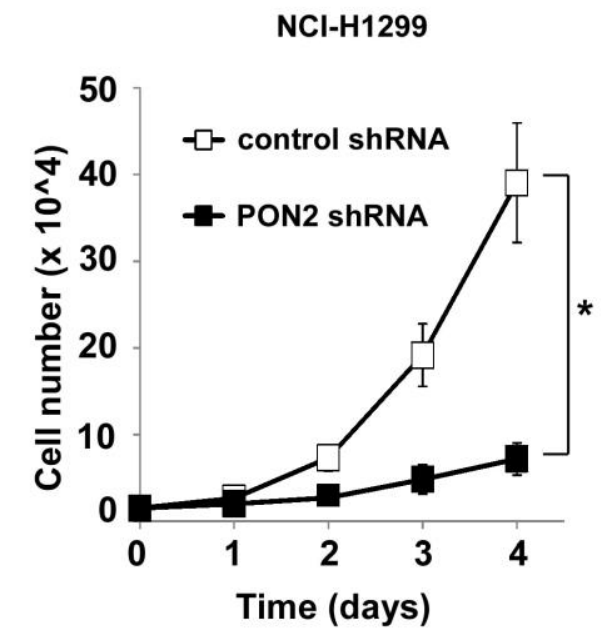

D

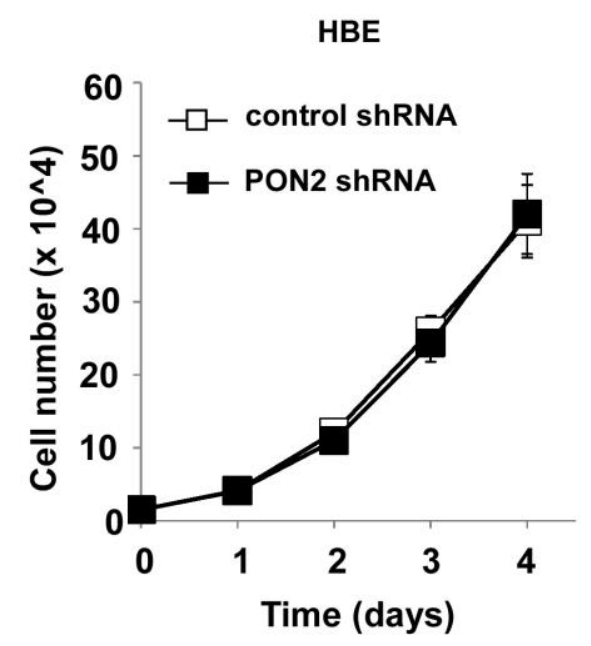


Figure 4.6. PON2 is essential for human lung tumor cell proliferation but not non-transformed cell proliferation. The proliferation of A549 cells (A), $\mathrm{NCl}-$ H1299 cells (B), HEK-293T cells (C) and HBE cells (D) expressing PON2 shRNA or the empty vector control was measured. Proliferation of $\mathrm{NCl}-\mathrm{H} 1299$ and A549 cells with reduced PON2 expression was slower compared with their counterparts expressing the empty vector. HEK-293T cells (C) and HBE cells with reduced PON2 expression proliferated at the same rate as their vector control counterparts. All data shown are mean \pm standard deviation of three independent experiments. Asterisks indicate $\mathrm{P}$ values of $<0.05\left(^{*}\right)$ by Student's unpaired t test. 
4.3.7. Deficiency in PON2 expression induces $\mathrm{G} 1$ cell cycle arrest of $A 549$ cells.

To investigate the effects of deficient PON2 expression, we performed cell cycle analysis to determine the cell cycle profile of A549 cells expressing PON2 shRNA or the empty vector. We found that deficiency in PON2 expression induces cell cycle arrest at G1 phase in A549 cells (Figure 4.7). 
A
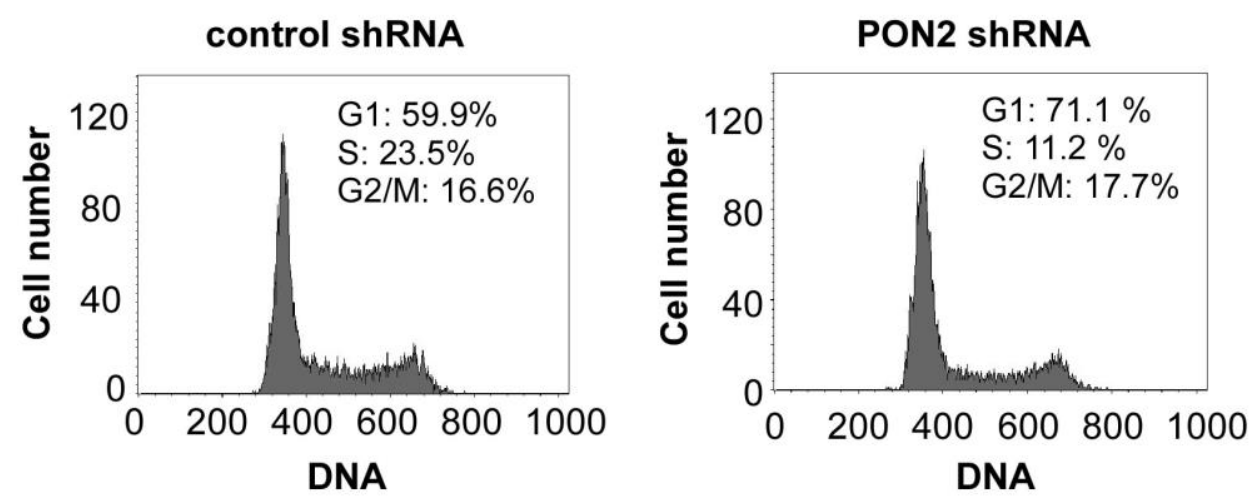

B

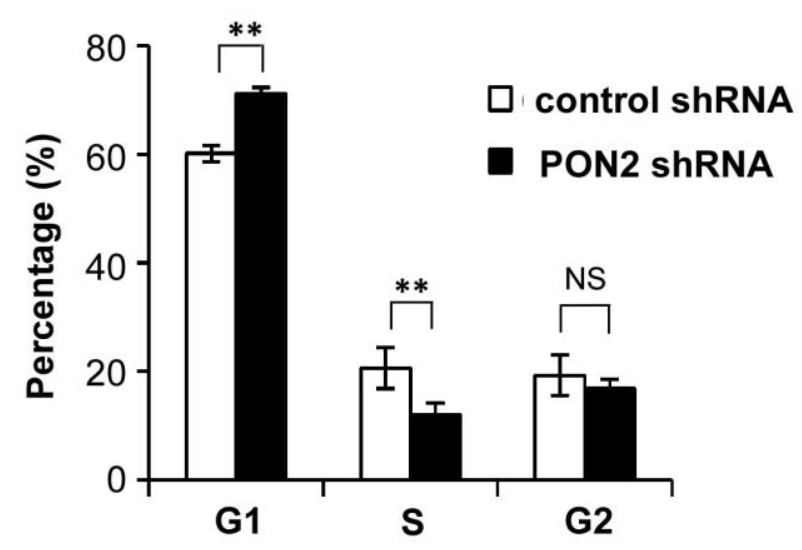

Figure 4.7. Deficiency in PON2 expression induces G1 cell cycle arrest of A549 cells. (A) Cell cycle profiles of A549 cells expressing PON2 shRNA or the empty vector control were determined. (B) Summary of the data shown in (A). Reducing PON2 expression caused higher percentage of the cells in G1 phase of cell cycle. All data shown are mean \pm standard deviation of three independent experiments. Asterisks indicate $\mathrm{P}$ values of $\left.<0.01{ }^{* *}\right)$ by Student's unpaired t test. "ns", no significance. 
4.3.8. PON2 interacts with components of mitochondrial electron transport chain. Since PON2 is known to be localized on mitochondrial inner membrane and probably cleave oxidized mitochondrial lipids $(73,181)$, we carried out a proteomics study to identify mitochondrial proteins associated with PON2 (Figure 4.8A). Mitochondria were isolated from A549 cells by a biochemical fractionation approach, and proteins bound to PON2 were acquired by immunoprecipitation using antibodies against PON2. Twelve mitochondrial proteins were discovered in protein complexes containing PON2, and several potential PON2-binding proteins are components of mitochondrial electron transport chain. Among them, a component of Complex II, succinate dehydrogenase complex flavoprotein subunit $A(S D H A)$, displayed highest specific binding to PON2 (Figure 4.8B-C). These data suggest that PON2 interacts with components of mitochondrial electron transport chain in NSCLC cells. 
A

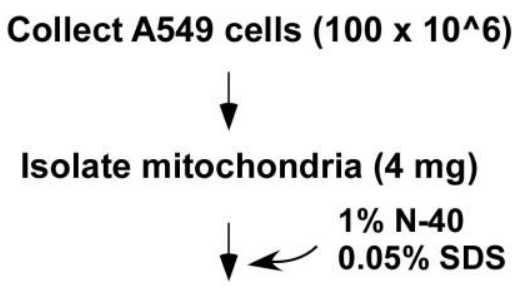

Generate mitochondria lysate<smiles>CCCCC[C@H](CCCC)OS(=O)(=O)O</smiles>

immunoprecipitation<smiles>[3H][3H]</smiles>

Proteomics analysis
B

\section{Succinate dehydrogenase}

tRNA (adenine(58)-N(1))-methyltransferase

Elongation factor Ts

Aldehyde dehydrogenase

Tricarboxylate transport protein

Methylcrotonoyl-CoA carboxylase subunit alpha

Glutamate dehydrogenase 1

Sulfide:quinone oxidoreductase

Calcium-binding mitochondrial carrier protein Aralar2

ATP synthase subunit delta

Acyl-coenzyme A thioesterase 9

$28 \mathrm{~S}$ ribosomal protein S34
C

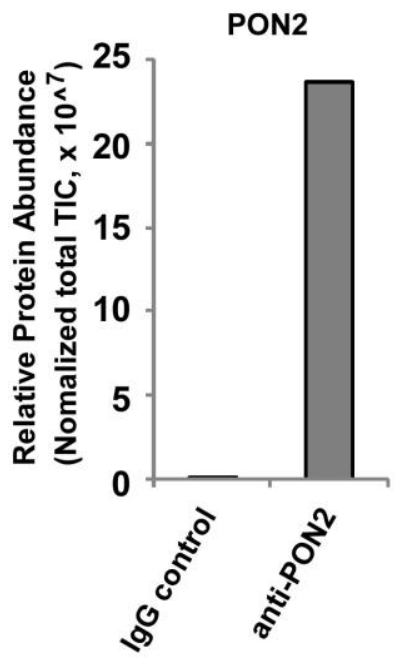

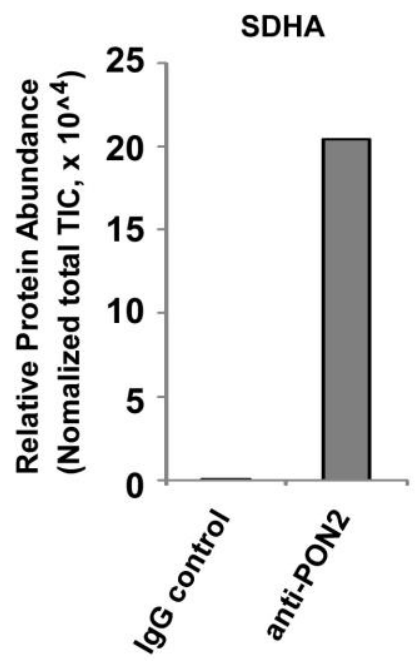

D

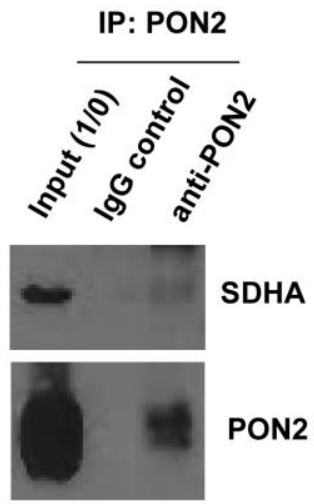


Figure 4.8. PON2 interacts with components of mitochondrial electron transport chain. (A) The depiction of immunoprecipitation study to identify mitochondrial proteins associated with PON2. (B) Twelve mitochondrial proteins were found in protein complexes containing PON2. (C) Relative abundance of PON2 and SDHA was evaluated by calculating normalized total TIC in a proteomics study. (D) The binding between PON2 and SDHA was detected by immunoprecipitation. 


\subsection{DISCUSSION}

Despite recent progress, the detailed mechanisms underlying the initiation and development of non-small NSCLC are still missing. Understanding of these mechanisms is essential for successful prevention, diagnosis and treatment of NSCLC. Here we report that PON2 is overexpressed in tumor tissues of NSCLC patients compared with corresponding adjacent normal tissues. Importantly, enhanced PON2 expression in NSCLC cells is essential for their proliferation, as reducing PON2 expression in NSCLC cells results in cell cycle arrest at the G1 phase and markedly slower proliferation. It is conceivable that enhanced PON2 expression in NSCLC cells sustain rapid cell proliferation, probably by influencing mitochondrial bioenergetics. Previous research of PON2 expression in tumor cells largely focuses on its effects on conferring resistance to chemotherapeutic drugs $(78,79,95)$. Our data for the first time demonstrate a critical role of PON2 in NSCLC cell proliferation.

As a lactonase/arylesterase with anti-oxidant properties, PON2 expression is elevated under a variety of physiological and pathological conditions. In mouse peritoneal macrophages, oxidative stress promotes PON2 expression at both mRNA and protein levels, but decreases PON3 expression (182). Similarly, enhanced reactive oxygen species (ROS) production in vascular cells evokes oxidative stress, which in turn elevates PON2 expression (183). During monocytes differentiation into macrophages, PON2 expression is increased through a mechanism dependent on activating nicotinamide-adenine- 
dinucleotide-phosphate (NADPH)-oxidase, which is partially modulated by the transcription factor AP-1 (184). A common theme arisen from these studies is that PON2 expression increase may represent a compensatory signaling cascade against the enhanced cellular oxidative stress, which is in agreement with the well-established anti-oxidative activity of PON2 $(73,78,179,181,183$, 185).

The level of oxidative stress within cells greatly influences many important cellular functions of mammalian cells (186). Emerging evidence supports the idea that a persistent and excessive production of intracellular ROS is involved in the initiation and progression of cancer (187-189). ROS function as cellular messengers in signaling cascades of cancer cells to initiate tumorigenesis and promote tumor development, consistent with their activity to evoke DNA damage and subsequent tumorigenic genetic mutations. Therefore, it is conceivable that PON2 expression may be elevated in cancer cells with high levels of oxidative stress to provide protection against ROS-induced damages. Indeed, enhanced PON2 mRNA levels have been observed in a variety of tumors through microarray studies (161-164). Subsequent experiments using tumor samples combined from patients or healthy people further validate the increased PON2 expression at the protein levels in some of those tumors (78). In this study, we directly compare PON2 expression at protein level in human NSCLC tumor tissues with neighboring normal tissues from the same patient (Figure 4.1). Our data provide unequivocal evidence that PON2 are overexpressed in NSCLC, 
which is critical for NSCLC cell proliferation but not for their untransformed counterparts (Figure 4.6). Although the signaling pathway utilized by NSCLC cells to achieve an increase in PON2 expression is still unclear, the underlying mechanisms is likely associated with elevated oxidative stress in cancer cells.

Rapidly proliferating tumor cells is known to consume high levels of glucose and produce lactate as major glucose-derived carbon in the presence of oxygen (termed the Warburg effect), which was originally thought to be caused by irreversible damages to mitochondrial oxidative metabolism and a subsequent compensatory increase in glycolysis (190). However, many highly proliferating tumor cells do not exhibit dysfunction in mitochondrial oxidative metabolism after careful studies $(191,192)$. Furthermore, oncogenic transformation of human mesenchymal stem cells promotes mitochondrial bioenergetics (193), and mitochondrial metabolism is essential for Kras-evoked tumor cell proliferation and tumorigenesis $(194,195)$. These findings demonstrate a critical role of mitochondrial oxidative metabolism in tumor cell proliferation. The observation of reduced electron transport chain activities and oxygen consumption in PON2deficient mice provides evidence that PON2 plays a critical role in maintaining mitochondrial respiratory chain $(73,196)$. Thus, a possible scenario is that overexpressed PON2 functions in NSCLC cells to promote mitochondrial oxidative metabolism to meet the metabolic demand for rapid proliferation of cells. 
Our proteomics studies demonstrate that PON2 likely interacts with components of mitochondrial electron transport chain (Figure 4.8). Among them, SDHA, a component of Complex II, displayed highest specific binding to PON2. In addition, PON2 also interacts with several subunits of the water-soluble catalytic sector (F1) of adenosine triphosphate (ATP) (Figure 4.8). Currently it remains unknown which component of electron transport chain functionally interacts with PON2, but our study clearly reveals an important function of PON2 in NSCLC cell proliferation. Future studies will focus on elucidating the detailed molecular mechanism of PON2 enhancing NSCLC cell proliferation. 


\section{CHAPTER V \\ CONCLUSION AND FUTURE DIRECTIONS}

\subsection{Summary and Conclusions}

Pseudomonas aeruginosa produces N-(3-oxododecanoyl)-homoserine lactone (C12) as a quorum-sensing molecule for bacteria-bacteria communication. C12 is known to influence many aspects of human host cell physiology, including induction of cell death. Further to this, $\mathrm{C} 12$ has been reported to induce apoptosis in various types of tumor cells. However, the detailed molecular mechanism of C12-triggerred tumor cell apoptosis remains unclear. Additionally, it is completely unknown whether $\mathrm{C} 12$ possesses any potential therapeutic effects in vivo. To clarify cell death signaling induced by $\mathrm{C} 12$, we examined mouse embryonic fibroblasts (MEFs) deficient in "initiator" caspases or "effector" caspases. Our data has indicated that $\mathrm{C} 12$ selectively induces the mitochondriadependent intrinsic apoptotic pathway by quickly triggering mitochondrial outer membrane permeabilization (MOMP). Importantly, the activities of $\mathrm{C} 12$ to permeabilize mitochondria is independent of activation of both "initiator" and "effector" caspases. Furthermore, C12 directly induces MOMP in vitro. Our studies have also indicated that, unlike most apoptotic inducers, C12 evokes a novel form of apoptosis in tumor cells independent of both pro- and anti-apoptotic $\mathrm{Bcl}-2$ proteins. We have also determined that $\mathrm{C} 12$ inhibits tumor 
growth in animals regardless of either pro- or anti-apoptotic Bcl-2 proteins. Furthermore, we have demonstrated that unlike conventional chemotherapeutics, C12 requires paraoxonase 2 (PON2), a lactonase/arylesterase with anti-oxidant properties, to exert its cytotoxicity on tumor cells. Our recent studies have found that PON2 is overexpressed in tumor tissues of non-small cell lung cancer (NSCLC) patients compared with corresponding adjacent normal tissues. Importantly, enhanced PON2 expression in NSCLC cells is essential for their proliferation, as reducing PON2 expression in NSCLC cells results in cell cycle arrest at the G1 phase and markedly slower proliferation. In contrast, altering PON2 expression fails to influence the proliferation of untransformed human epithelial cells, including bronchial epithelial cells. Using proteomics and biochemical approaches, we have shown that PON2 in NSCLC cells specifically interacts with succinate dehydrogenase complex flavoprotein subunit A (SDHA), a component of Complex II of mitochondrial electron transport chain. Overall, our studies have discovered a mitochondrial apoptotic-signaling pathway triggered by $\mathrm{C} 12$, in which $\mathrm{C} 12$ or its metabolite(s) acts to permeabilize mitochondria, leading to activation of apoptosis. Moreover, our results demonstrate that $\mathrm{C} 12$ inhibits tumor growth independent of both pro- and antiapoptotic Bcl-2 proteins via the induction of unique apoptotic-signaling mediated by PON2 in tumor cells. Enhanced PON2 expression in NSCLC cells might promote mitochondrial bioenergetics to sustain the rapid proliferation of NSCLC cells. 


\subsection{Future Perspectives}

We will investigate the mechanism of apoptosis mediated by PON2/C12 interaction. $\mathrm{C} 12$ is preferentially toxic to cells expressing PON2. PON2 is known to function as a lactonase to cleave $\mathrm{C} 12$. We postulate that PON2 hydrolyzes C12 in cells, generating a pro-apoptotic molecule to induce Bcl-2 proteinindependent apoptosis.

We will also Investigate the mechanism of PON2 in modulating lung tumor cell proliferation.PON2 is overexpressed in tumor tissues from NSCLC patients. PON2 expression is important for the proliferation of NSCLC cells but not for their untransformed counterparts. Therefore, we propose that enhanced PON2 expression in NSCLC cells promotes mitochondrial bioenergetics which subsequently generates enough ATP and metabolites to sustain the rapid NSCLC cell proliferation. 


\section{REFERENCES}

1. Cory S and Adams JM (2002) The Bcl2 family: regulators of the cellular life-or-death switch. Nat. Rev. Cancer 2:647-656

2. Hardwick JM and Youle RJ (2009) SnapShot: BCL-2 proteins. Cell 138:404, 404

3. Adams JM and Cory S (2007) Bcl-2-regulated apoptosis: mechanism and therapeutic potential. Curr. Opin. Immunol. 19:488-496

4. Fesik SW (2005) Promoting apoptosis as a strategy for cancer drug discovery. Nat. Rev. Cancer 5:876-885

5. Chipuk JE and Green DR (2008) How do BCL-2 proteins induce mitochondrial outer membrane permeabilization? Trends Cell Biol. 18:157-164

6. Irie $Y$ and Parsek MR (2008) Quorum sensing and microbial biofilms. Curr. Top. Microbiol. Immunol. 322:67-84

7. Zhao G, Neely AM, Schwarzer C, Lu H, Whitt AG, Stivers NS, Burlison JA, White C, Machen TE and Li C (2016) N-(3-oxo-acyl) homoserine lactone inhibits tumor growth independent of Bcl-2 proteins. Oncotarget.

8. Schwarzer C, Fu Z, Morita T, Whitt AG, Neely AM, Li C and Machen TE (2015) Paraoxonase 2 serves a proapopotic function in mouse and human cells in response to the Pseudomonas aeruginosa quorumsensing molecule $\mathrm{N}$-(3-Oxododecanoyl)-homoserine lactone. J. Biol. Chem. 290:7247-7258

9. Reed JC (1999) Dysregulation of apoptosis in cancer. J. Clin. Oncol. $17: 2941-2953$

10. Vaux DL and Korsmeyer SJ (1999) Cell death in development. Cell 96:245-254

11. Ziegler $U$ and Groscurth $P$ (2004) Morphological features of cell death. News Physiol Sci. 19:124-128

12. Fuchs $\mathrm{Y}$ and Steller $\mathrm{H}$ (2011) Programmed cell death in animal development and disease. Cell 147:742-758 
13. Cory S, Huang DC and Adams JM (2003) The Bcl-2 family: roles in cell survival and oncogenesis. Oncogene 22:8590-8607

14. Tibbetts MD, Zheng $L$ and Lenardo MJ (2003) The death effector domain protein family: regulators of cellular homeostasis. Nat. Immunol. 4:404-409

15. Jenner $P$ (2003) Oxidative stress in Parkinson's disease. Ann. Neurol. 53 Suppl 3:S26-S36

16. Upham BL and Wagner JG (2001) Toxicant-induced oxidative stress in cancer. Toxicol. Sci. 64:1-3

17. Petros AM, Olejniczak ET and Fesik SW (2004) Structural biology of the Bcl-2 family of proteins. Biochim. Biophys. Acta 1644:83-94

18. Petros AM, Olejniczak ET and Fesik SW (2004) Structural biology of the Bcl-2 family of proteins. Biochim. Biophys. Acta 1644:83-94

19. Fadeel B and Orrenius S (2005) Apoptosis: a basic biological phenomenon with wide-ranging implications in human disease. J. Intern. Med. 258:479-517

20. Ellis RE, Yuan JY and Horvitz HR (1991) Mechanisms and functions of cell death. Annu. Rev. Cell Biol. 7:663-698

21. Xu D, Li Y, Arcaro M, Lackey M and Bergmann A (2005) The CARDcarrying caspase Dronc is essential for most, but not all, developmental cell death in Drosophila. Development 132:21252134

22. Gelbard HA, Boustany RM and Schor NF (1997) Apoptosis in development and disease of the nervous system: II. Apoptosis in childhood neurologic disease. Pediatr. Neurol. 16:93-97

23. Gilbert SF (2003) Opening Darwin's black box: teaching evolution through developmental genetics. Nat. Rev. Genet. 4:735-741

24. Fairweather D, Kaya Z, Shellam GR, Lawson CM and Rose NR (2001) From infection to autoimmunity. J. Autoimmun. 16:175-186

25. Renehan AG, Booth C and Potten CS (2001) What is apoptosis, and why is it important? BMJ 322:1536-1538

26. King C, llic A, Koelsch K and Sarvetnick N (2004) Homeostatic expansion of $T$ cells during immune insufficiency generates autoimmunity. Cell 117:265-277 
27. Coultas $L$ and Strasser A (2003) The role of the Bcl-2 protein family in cancer. Semin. Cancer Biol. 13:115-123

28. Henry-Mowatt J, Dive C, Martinou JC and James D (2004) Role of mitochondrial membrane permeabilization in apoptosis and cancer. Oncogene 23:2850-2860

29. Srinivasula SM, Datta P, Fan XJ, Fernandes-Alnemri T, Huang $Z$ and Alnemri ES (2000) Molecular determinants of the caspasepromoting activity of Smac/DIABLO and its role in the death receptor pathway. J. Biol. Chem. 275:36152-36157

30. Wajant $H(2002)$ The Fas signaling pathway: more than a paradigm. Science 296:1635-1636

31. Boldin MP, Varfolomeev EE, Pancer Z, Mett IL, Camonis JH and Wallach $D$ (1995) A novel protein that interacts with the death domain of Fas/APO1 contains a sequence motif related to the death domain. J. Biol. Chem. 270:7795-7798

32. Chinnaiyan AM, O'Rourke K, Tewari M and Dixit VM (1995) FADD, a novel death domain-containing protein, interacts with the death domain of Fas and initiates apoptosis. Cell 81:505-512

33. Wang J, Chun HJ, Wong W, Spencer DM and Lenardo MJ (2001) Caspase-10 is an initiator caspase in death receptor signaling. Proc. Natl. Acad. Sci. U. S. A 98:13884-13888

34. Bao Q and Shi $Y$ (2007) Apoptosome: a platform for the activation of initiator caspases. Cell Death. Differ. 14:56-65

35. Ashkenazi A (2002) Targeting death and decoy receptors of the tumournecrosis factor superfamily. Nat. Rev. Cancer 2:420-430

36. Fulda S and Debatin KM (2006) Extrinsic versus intrinsic apoptosis pathways in anticancer chemotherapy. Oncogene 25:4798-4811

37. Boatright KM and Salvesen GS (2003) Mechanisms of caspase activation. Curr. Opin. Cell Biol. 15:725-731

38. Saraste A and Pulkki K (2000) Morphologic and biochemical hallmarks of apoptosis. Cardiovasc. Res. 45:528-537

39. Shi Y (2004) Caspase activation: revisiting the induced proximity model. Cell 117:855-858 
40. Lamkanfi M, Festjens N, Declercq W, Vanden Berghe T and Vandenabeele P (2007) Caspases in cell survival, proliferation and differentiation. Cell Death. Differ. 14:44-55

41. Knezevich S, Ludkovski O, Salski C, Lestou V, Chhanabhai M, Lam W, Klasa R, Connors JM, Dyer MJ, Gascoyne RD and Horsman DE (2005) Concurrent translocation of BCL2 and MYC with a single immunoglobulin locus in high-grade B-cell lymphomas. Leukemia 19:659-663

42. Youle RJ and Strasser A (2008) The BCL-2 protein family: opposing activities that mediate cell death. Nat. Rev. Mol. Cell Biol. 9:47-59

43. Tsujimoto $Y(2003)$ Cell death regulation by the Bcl-2 protein family in the mitochondria. J. Cell Physiol 195:158-167

44. Hsu SY, Kaipia A, McGee E, Lomeli M and Hsueh AJ (1997) Bok is a proapoptotic $\mathrm{Bcl}-2$ protein with restricted expression in reproductive tissues and heterodimerizes with selective anti-apoptotic Bcl-2 family members. Proc. Natl. Acad. Sci. U. S. A 94:12401-12406

45. Antonsson B, Montessuit S, Sanchez B and Martinou JC (2001) Bax is present as a high molecular weight oligomer/complex in the mitochondrial membrane of apoptotic cells. J. Biol. Chem. 276:11615-11623

46. Hsu YT, Wolter KG and Youle RJ (1997) Cytosol-to-membrane redistribution of $\mathrm{Bax}$ and $\mathrm{Bcl}-\mathrm{X}(\mathrm{L})$ during apoptosis. Proc. Natl. Acad. Sci. U. S. A 94:3668-3672

47. Mikhailov V, Mikhailova M, Degenhardt K, Venkatachalam MA, White E and Saikumar P (2003) Association of Bax and Bak homooligomers in mitochondria. Bax requirement for Bak reorganization and cytochrome c release. J. Biol. Chem. 278:5367-5376

48. Glick D, Barth S and Macleod KF (2010) Autophagy: cellular and molecular mechanisms. J. Pathol. 221:3-12

49. Saftig P, Beertsen W and Eskelinen EL (2008) LAMP-2: a control step for phagosome and autophagosome maturation. Autophagy. 4:510512

50. Glick D, Barth S and Macleod KF (2010) Autophagy: cellular and molecular mechanisms. J. Pathol. 221:3-12

51. Eskelinen EL (2008) To be or not to be? Examples of incorrect identification of autophagic compartments in conventional 
transmission electron microscopy of mammalian cells. Autophagy. 4:257-260

52. Kroemer G, El-Deiry WS, Golstein P, Peter ME, Vaux D, Vandenabeele P, Zhivotovsky B, Blagosklonny MV, Malorni W, Knight RA, Piacentini M, Nagata S and Melino G (2005) Classification of cell death: recommendations of the Nomenclature Committee on Cell Death. Cell Death. Differ. 12 Suppl 2:1463-1467

53. Edinger AL and Thompson CB (2004) Death by design: apoptosis, necrosis and autophagy. Curr. Opin. Cell Biol. 16:663-669

54. Festjens $N$, Vanden Berghe $T$ and Vandenabeele $P$ (2006) Necrosis, a well-orchestrated form of cell demise: signalling cascades, important mediators and concomitant immune response. Biochim. Biophys. Acta 1757:1371-1387

55. Zong WX and Thompson CB (2006) Necrotic death as a cell fate. Genes Dev. 20:1-15

56. Yuan J (2006) Divergence from a dedicated cellular suicide mechanism: exploring the evolution of cell death. Mol. Cell 23:1-12

57. Zitvogel L, Casares N, Pequignot MO, Chaput N, Albert ML and Kroemer G (2004) Immune response against dying tumor cells. Adv. Immunol. 84:131-179

58. Miller MB and Bassler BL (2001) Quorum sensing in bacteria. Annu. Rev. Microbiol. 55:165-199

59. Schuster M and Greenberg EP (2006) A network of networks: quorumsensing gene regulation in Pseudomonas aeruginosa. Int. J. Med. Microbiol. 296:73-81

60. Kravchenko VV and Kaufmann GF (2013) Bacterial inhibition of inflammatory responses via TLR-independent mechanisms. Cell Microbiol. 15:527-536

61. Telford G, Wheeler D, Williams P, Tomkins PT, Appleby P, Sewell H, Stewart GS, Bycroft BW and Pritchard DI (1998) The Pseudomonas aeruginosa quorum-sensing signal molecule N-(3oxododecanoyl)-L-homoserine lactone has immunomodulatory activity. Infect. Immun. 66:36-42

62. Kravchenko VV, Kaufmann GF, Mathison JC, Scott DA, Katz AZ, Grauer DC, Lehmann M, Meijler MM, Janda KD and Ulevitch RJ (2008) Modulation of gene expression via disruption of NF-kappaB signaling by a bacterial small molecule. Science 321:259-263 
63. Valentine CD, Zhang H, Phuan PW, Nguyen J, Verkman AS and Haggie PM (2014) Small molecule screen yields inhibitors of Pseudomonas homoserine lactone-induced host responses. Cell Microbiol. 16:114

64. Grabiner MA, Fu Z, Wu T, Barry KC, Schwarzer C and Machen TE (2014) Pseudomonas aeruginosa quorum-sensing molecule homoserine lactone modulates inflammatory signaling through PERK and elF2alpha. J. Immunol. 193:1459-1467

65. Schwarzer C, Fu Z, Patanwala M, Hum L, Lopez-Guzman M, Illek B, Kong W, Lynch SV and Machen TE (2012) Pseudomonas aeruginosa biofilm-associated homoserine lactone $\mathrm{C} 12$ rapidly activates apoptosis in airway epithelia. Cell Microbiol. 14:698-709

66. Li L, Hooi D, Chhabra SR, Pritchard D and Shaw PE (2004) Bacterial Nacylhomoserine lactone-induced apoptosis in breast carcinoma cells correlated with down-modulation of STAT3. Oncogene 23:4894-4902

67. Shiner EK, Terentyev D, Bryan A, Sennoune S, Martinez-Zaguilan R, Li G, Gyorke S, Williams SC and Rumbaugh KP (2006) Pseudomonas aeruginosa autoinducer modulates host cell responses through calcium signalling. Cell Microbiol. 8:1601-1610

68. Valentine CD, Anderson MO, Papa FR and Haggie PM (2013) X-box binding protein 1 (XBP1s) is a critical determinant of Pseudomonas aeruginosa homoserine lactone-mediated apoptosis. PLoS. Pathog. 9:e1003576

69. Oliver CM, Schaefer AL, Greenberg EP and Sufrin JR (2009) Microwave synthesis and evaluation of phenacylhomoserine lactones as anticancer compounds that minimally activate quorum sensing pathways in Pseudomonas aeruginosa. J. Med. Chem. 52:15691575

70. Kumar AS, Bryan JN and Kumar SR (2014) Bacterial quorum sensing molecule N-3-oxo-dodecanoyl-L-homoserine lactone causes direct cytotoxicity and reduced cell motility in human pancreatic carcinoma cells. PLoS. One. 9:e106480

71. Dolnick R, Wu Q, Angelino NJ, Stephanie LV, Chow KC, Sufrin JR and Dolnick BJ (2005) Enhancement of 5-fluorouracil sensitivity by an rTS signaling mimic in $\mathrm{H} 630$ colon cancer cells. Cancer Res. 65:5917-5924

72. Schweikert EM, Amort J, Wilgenbus P, Forstermann U, Teiber JF and Horke S (2012) Paraoxonases-2 and -3 Are Important Defense 
Enzymes against Pseudomonas aeruginosa Virulence Factors due to Their Anti-Oxidative and Anti-Inflammatory Properties. J. Lipids 2012:352857

73. Devarajan A, Bourquard N, Hama S, Navab M, Grijalva VR, Morvardi S, Clarke CF, Vergnes L, Reue K, Teiber JF and Reddy ST (2011)

Paraoxonase 2 deficiency alters mitochondrial function and exacerbates the development of atherosclerosis. Antioxid. Redox. Signal. 14:341-351

74. Horke S, Xiao J, Schutz EM, Kramer GL, Wilgenbus P, Witte I, Selbach M and Teiber JF (2015) Novel Paraoxonase 2-Dependent Mechanism Mediating the Biological Effects of the Pseudomonas aeruginosa Quorum-Sensing Molecule N-(3-Oxo-Dodecanoyl)-I-Homoserine Lactone. Infect. Immun. 83:3369-3380

75. Chun CK, Ozer EA, Welsh MJ, Zabner J and Greenberg EP (2004) Inactivation of a Pseudomonas aeruginosa quorum-sensing signal by human airway epithelia. Proc. Natl. Acad. Sci. U. S. A 101:35873590

76. Draganov DI, Teiber JF, Speelman A, Osawa Y, Sunahara R and La Du BN (2005) Human paraoxonases (PON1, PON2, and PON3) are lactonases with overlapping and distinct substrate specificities. J. Lipid Res. 46:1239-1247

77. Stoltz DA, Ozer EA, Ng CJ, Yu JM, Reddy ST, Lusis AJ, Bourquard N, Parsek MR, Zabner J and Shih DM (2007) Paraoxonase-2 deficiency enhances Pseudomonas aeruginosa quorum sensing in murine tracheal epithelia. Am. J. Physiol Lung Cell Mol. Physiol 292:L852-L860

78. Witte I, Altenhofer S, Wilgenbus P, Amort J, Clement AM, Pautz A, Li H, Forstermann $U$ and Horke $S$ (2011) Beyond reduction of atherosclerosis: PON2 provides apoptosis resistance and stabilizes tumor cells. Cell Death. Dis. 2:e112

79. Witte I, Foerstermann U, Devarajan A, Reddy ST and Horke S (2012) Protectors or Traitors: The Roles of PON2 and PON3 in Atherosclerosis and Cancer. J. Lipids 2012:342806

80. Cross A, Allen JR, Burke J, Ducel G, Harris A, John J, Johnson D, Lew M, MacMillan B, Meers P and . (1983) Nosocomial infections due to Pseudomonas aeruginosa: review of recent trends. Rev. Infect. Dis. 5 Suppl 5:S837-S845

81. Gaspar MC, Couet W, Olivier JC, Pais AA and Sousa JJ (2013) Pseudomonas aeruginosa infection in cystic fibrosis lung disease 
and new perspectives of treatment: a review. Eur. J. Clin. Microbiol. Infect. Dis. 32:1231-1252

82. Hughes DT and Sperandio V (2008) Inter-kingdom signalling: communication between bacteria and their hosts. Nat. Rev. Microbiol. 6:111-120

83. Shiner EK, Rumbaugh KP and Williams SC (2005) Inter-kingdom signaling: deciphering the language of acyl homoserine lactones. FEMS Microbiol. Rev. 29:935-947

84. Rumbaugh KP (2007) Convergence of hormones and autoinducers at the host/pathogen interface. Anal. Bioanal. Chem. 387:425-435

85. Chambers CE, Visser MB, Schwab U and Sokol PA (2005) Identification of $\mathrm{N}$-acylhomoserine lactones in mucopurulent respiratory secretions from cystic fibrosis patients. FEMS Microbiol. Lett. 244:297-304

86. Charlton TS, de NR, Netting A, Kumar N, Hentzer M, Givskov M and Kjelleberg S (2000) A novel and sensitive method for the quantification of N-3-oxoacyl homoserine lactones using gas chromatography-mass spectrometry: application to a model bacterial biofilm. Environ. Microbiol. 2:530-541

87. Fu Z, Bettega K, Carroll S, Buchholz KR and Machen TE (2007) Role of $\mathrm{Ca} 2+$ in responses of airway epithelia to Pseudomonas aeruginosa, flagellin, ATP, and thapsigargin. Am. J. Physiol Lung Cell Mol. Physiol 292:L353-L364

88. Schwarzer C, Wong S, Shi J, Matthes E, Illek B, lanowski JP, Arant RJ, Isacoff E, Vais H, Foskett JK, Maiellaro I, Hofer AM and Machen TE (2010) Pseudomonas aeruginosa Homoserine lactone activates store-operated CAMP and cystic fibrosis transmembrane regulatordependent $\mathrm{Cl}$ - secretion by human airway epithelia. J. Biol. Chem. 285:34850-34863

89. Taguchi R, Tanaka S, Joe GH, Maseda H, Nomura N, Ohnishi J, Ishizuka $\mathrm{S}$, Shimizu $\mathrm{H}$ and Miyazaki $\mathrm{H}$ (2014) Mucin 3 is involved in intestinal epithelial cell apoptosis via N-(3-oxododecanoyl)-Lhomoserine lactone-induced suppression of Akt phosphorylation. Am. J. Physiol Cell Physiol 307:C162-C168

90. Valentine CD, Anderson MO, Papa FR and Haggie PM (2013) X-box binding protein 1 (XBP1s) is a critical determinant of Pseudomonas aeruginosa homoserine lactone-mediated apoptosis. PLoS. Pathog. 9:e1003576 
91. Kravchenko VV, Kaufmann GF, Mathison JC, Scott DA, Katz AZ, Wood MR, Brogan AP, Lehmann M, Mee JM, Iwata K, Pan Q, Fearns C, Knaus UG, Meijler MM, Janda KD and Ulevitch RJ (2006) N-(3-oxoacyl)homoserine lactones signal cell activation through a mechanism distinct from the canonical pathogen-associated molecular pattern recognition receptor pathways. J. Biol. Chem. $281: 28822-28830$

92. Li J and Yuan J (2008) Caspases in apoptosis and beyond. Oncogene 27:6194-6206

93. Schwarzer C, Fu Z, Shuai S, Babbar S, Zhao G, Li C and Machen TE (2014) Pseudomonas aeruginosa homoserine lactone triggers apoptosis and Bak/Bax-independent release of mitochondrial cytochrome C in fibroblasts. Cell Microbiol. 16:1094-1104

94. Jacobi CA, Schiffner F, Henkel M, Waibel M, Stork B, Daubrawa M, Eberl L, Gregor M and Wesselborg S (2009) Effects of bacterial N-acyl homoserine lactones on human Jurkat T lymphocytes-OdDHL induces apoptosis via the mitochondrial pathway. Int. J. Med. Microbiol. 299:509-519

95. Zhao G, Neely AM, Schwarzer C, Lu H, Whitt AG, Stivers NS, Burlison JA, White C, Machen TE and Li C (2016) N-(3-oxo-acyl) homoserine lactone inhibits tumor growth independent of Bcl-2 proteins. Oncotarget.

96. Horikawa M, Tateda K, Tuzuki E, Ishii Y, Ueda C, Takabatake T, Miyairi S, Yamaguchi K and Ishiguro M (2006) Synthesis of Pseudomonas quorum-sensing autoinducer analogs and structural entities required for induction of apoptosis in macrophages. Bioorg. Med. Chem. Lett. 16:2130-2133

97. Tateda K, Ishii Y, Horikawa M, Matsumoto T, Miyairi S, Pechere JC, Standiford TJ, Ishiguro M and Yamaguchi K (2003) The Pseudomonas aeruginosa autoinducer N-3-oxododecanoyl homoserine lactone accelerates apoptosis in macrophages and neutrophils. Infect. Immun. 71:5785-5793

98. Schwarzer C, Fu Z, Shuai S, Babbar S, Zhao G, Li C and Machen TE (2014) Pseudomonas aeruginosa homoserine lactone triggers apoptosis and Bak/Bax-independent release of mitochondrial cytochrome C in fibroblasts. Cell Microbiol. 16:1094-1104

99. Zhao G, Lu H and Li C (2015) Proapoptotic activities of protein disulfide isomerase (PDI) and PDIA3 protein, a role of the Bcl-2 protein Bak. J. Biol. Chem. 290:8949-8963 
100. Goldstein JC, Munoz-Pinedo C, Ricci JE, Adams SR, Kelekar A, Schuler M, Tsien RY and Green DR (2005) Cytochrome $c$ is released in a single step during apoptosis. Cell Death. Differ. 12:453-462

101. Goldstein JC, Waterhouse NJ, Juin P, Evan GI and Green DR (2000) The coordinate release of cytochrome $c$ during apoptosis is rapid, complete and kinetically invariant. Nat. Cell Biol. 2:156-162

102. Wang X, Olberding KE, White $C$ and Li C (2011) Bcl-2 proteins regulate ER membrane permeability to luminal proteins during ER stressinduced apoptosis. Cell Death. Differ. 18:38-47

103. Danial NN and Korsmeyer SJ (2004) Cell death: critical control points. Cell 116:205-219

104. Lakhani SA, Masud A, Kuida K, Porter GA, Jr., Booth CJ, Mehal WZ, Inayat I and Flavell RA (2006) Caspases 3 and 7: key mediators of mitochondrial events of apoptosis. Science 311:847-851

105. Newmeyer DD and Ferguson-Miller S (2003) Mitochondria: releasing power for life and unleashing the machineries of death. Cell $112: 481-490$

106. Tait SW and Green DR (2010) Mitochondria and cell death: outer membrane permeabilization and beyond. Nat. Rev. Mol. Cell Biol. $11: 621-632$

107. Schwarzer C, Ravishankar B, Patanwala M, Shuai S, Fu Z, Illek B, Fischer $\mathrm{H}$ and Machen TE (2014) Thapsigargin blocks Pseudomonas aeruginosa homoserine lactone-induced apoptosis in airway epithelia. Am. J. Physiol Cell Physiol 306:C844-C855

108. Beisner DR, Ch'en IL, Kolla RV, Hoffmann A and Hedrick SM (2005) Cutting edge: innate immunity conferred by $B$ cells is regulated by caspase-8. J. Immunol. 175:3469-3473

109. Karbowski M and Youle RJ (2003) Dynamics of mitochondrial morphology in healthy cells and during apoptosis. Cell Death. Differ. 10:870-880

110. Dagda RK, Cherra SJ, III, Kulich SM, Tandon A, Park D and Chu CT (2009) Loss of PINK1 function promotes mitophagy through effects on oxidative stress and mitochondrial fission. J. Biol. Chem. 284:13843-13855

111. Boya P, Andreau K, Poncet D, Zamzami N, Perfettini JL, Metivier D, Ojcius DM, Jaattela M and Kroemer G (2003) Lysosomal membrane permeabilization induces cell death in a mitochondriondependent fashion. J. Exp. Med. 197:1323-1334 
112. Kurz T, Gustafsson B and Brunk UT (2006) Intralysosomal iron chelation protects against oxidative stress-induced cellular damage. FEBS $\mathrm{J}$. 273:3106-3117

113. Berndt C, Kurz T, Selenius M, Fernandes AP, Edgren MR and Brunk UT (2010) Chelation of lysosomal iron protects against ionizing radiation. Biochem. J. 432:295-301

114. Schwarzer C, Fu Z, Morita T, Whitt AG, Neely AM, Li C and Machen TE (2015) Paraoxonase 2 serves a proapopotic function in mouse and human cells in response to the Pseudomonas aeruginosa quorumsensing molecule $\mathrm{N}$-(3-Oxododecanoyl)-homoserine lactone. J. Biol. Chem. 290:7247-7258

115. Horke S, Xiao J, Schutz EM, Kramer GL, Wilgenbus P, Witte I, Selbach M and Teiber JF (2015) Novel Paraoxonase 2-Dependent Mechanism Mediating the Biological Effects of the Pseudomonas aeruginosa Quorum-Sensing Molecule N-(3-Oxo-Dodecanoyl)-L-Homoserine Lactone. Infect. Immun. 83:3369-3380

116. Wei MC, Zong WX, Cheng EH, Lindsten T, Panoutsakopoulou V, Ross AJ, Roth KA, MacGregor GR, Thompson CB and Korsmeyer SJ (2001) Proapoptotic BAX and BAK: a requisite gateway to mitochondrial dysfunction and death. Science 292:727-730

117. Zong WX, Li C, Hatzivassiliou G, Lindsten T, Yu QC, Yuan J and Thompson CB (2003) Bax and Bak can localize to the endoplasmic reticulum to initiate apoptosis. J. Cell Biol. 162:59-69

118. Hagmann H, Kuczkowski A, Ruehl M, Lamkemeyer T, Brodesser S, Horke S, Dryer S, Schermer B, Benzing T and Brinkkoetter PT (2014) Breaking the chain at the membrane: paraoxonase 2 counteracts lipid peroxidation at the plasma membrane. FASEB J. 28:17691779

119. Rosenblat M, Coleman R, Reddy ST and Aviram M (2009) Paraoxonase 2 attenuates macrophage triglyceride accumulation via inhibition of diacylglycerol acyltransferase 1. J. Lipid Res. 50:870-879

120. Meilin E, Aviram M and Hayek T (2010) Paraoxonase 2 (PON2) decreases high glucose-induced macrophage triglycerides (TG) accumulation, via inhibition of NADPH-oxidase and DGAT1 activity: studies in PON2-deficient mice. Atherosclerosis 208:390-395

121. Evan GI and Vousden KH (2001) Proliferation, cell cycle and apoptosis in cancer. Nature 411:342-348 
122. Yip KW and Reed JC (2008) Bcl-2 family proteins and cancer. Oncogene 27:6398-6406

123. Williams MM and Cook RS (2015) Bcl-2 family proteins in breast development and cancer: could Mcl-1 targeting overcome therapeutic resistance? Oncotarget. 6:3519-3530

124. Ertel F, Nguyen M, Roulston A and Shore GC (2013) Programming cancer cells for high expression levels of Mcl1. EMBO Rep. 14:328-336

125. Sturm I, Petrowsky H, Volz R, Lorenz M, Radetzki S, Hillebrand T, Wolff G, Hauptmann S, Dorken B and Daniel PT (2001) Analysis of p53/BAX/p16(ink4a/CDKN2) in esophageal squamous cell carcinoma: high BAX and p16(ink4a/CDKN2) identifies patients with good prognosis. J. Clin. Oncol. 19:2272-2281

126. Sturm I, Petrowsky H, Volz R, Lorenz M, Radetzki S, Hillebrand T, Wolff G, Hauptmann S, Dorken B and Daniel PT (2001) Analysis of p53/BAX/p16(ink4a/CDKN2) in esophageal squamous cell carcinoma: high BAX and p16(ink4a/CDKN2) identifies patients with good prognosis. J. Clin. Oncol. 19:2272-2281

127. Tai YT, Lee S, Niloff E, Weisman C, Strobel T and Cannistra SA (1998) BAX protein expression and clinical outcome in epithelial ovarian cancer. J. Clin. Oncol. 16:2583-2590

128. Hallgren $O$, Gustafsson L, Irjala $H$, Selivanova $G$, Orrenius $S$ and Svanborg C (2006) HAMLET triggers apoptosis but tumor cell death is independent of caspases, Bcl-2 and p53. Apoptosis. $11: 221-233$

129. Anether G, Tinhofer I, Senfter M and Greil R (2003) Tetrocarcin-A-induced ER stress mediates apoptosis in B-CLL cells via a Bcl-2-independent pathway. Blood 101:4561-4568

130. Yue W, Zheng X, Lin Y, Yang CS, Xu Q, Carpizo D, Huang H, DiPaola RS and Tan XL (2015) Metformin combined with aspirin significantly inhibit pancreatic cancer cell growth in vitro and in vivo by suppressing anti-apoptotic proteins $\mathrm{Mcl}-1$ and $\mathrm{Bcl}-2$. Oncotarget. 6:21208-21224

131. Eno CO, Zhao G, Venkatanarayan A, Wang B, Flores ER and Li C (2013) Noxa couples lysosomal membrane permeabilization and apoptosis during oxidative stress. Free Radic. Biol. Med. 65:26-37

132. Giard DJ, Aaronson SA, Todaro GJ, Arnstein P, Kersey JH, Dosik H and Parks WP (1973) In vitro cultivation of human tumors: 
establishment of cell lines derived from a series of solid tumors. J. Natl. Cancer Inst. 51:1417-1423

133. Huang H, Shah K, Bradbury NA, Li C and White C (2014) Mcl-1 promotes lung cancer cell migration by directly interacting with VDAC to increase mitochondrial $\mathrm{Ca} 2+$ uptake and reactive oxygen species generation. Cell Death. Dis. 5:e1482

134. Zhao G, Lu H and Li C (2015) Proapoptotic activities of protein disulfide isomerase (PDI) and PDIA3 protein, a role of the Bcl-2 protein Bak. J. Biol. Chem. 290:8949-8963

135. Wang $C$ and Youle RJ (2012) Predominant requirement of Bax for apoptosis in HCT116 cells is determined by Mcl-1's inhibitory effect on Bak. Oncogene 31:3177-3189

136. Zhao G, Wang J, Wang X, Chen S, Zhao Y, Gu F, Xu A and Wu L (2011) Mutagenicity of PFOA in mammalian cells: role of mitochondriadependent reactive oxygen species. Environ. Sci. Technol. 45:1638-1644

137. Zhao G, Zhu Y, Eno CO, Liu Y, Deleeuw L, Burlison JA, Chaires JB, Trent JO and Li C (2014) Activation of the proapoptotic Bcl-2 protein Bax by a small molecule induces tumor cell apoptosis. Mol. Cell Biol. 34:1198-1207

138. Schwarzer C, Fu Z, Morita T, Whitt AG, Neely AM, Li C and Machen TE (2015) Paraoxonase 2 serves a proapopotic function in mouse and human cells in response to the Pseudomonas aeruginosa quorumsensing molecule $\mathrm{N}$-(3-Oxododecanoyl)-homoserine lactone. J. Biol. Chem. 290:7247-7258

139. Lu H, Lu Q, Zheng Y and Li Q (2012) Notch signaling promotes the corneal epithelium wound healing. Mol. Vis. 18:403-411

140. Chai H, Hazawa M, Shirai N, Igarashi J, Takahashi K, Hosokawa Y, Suga $\mathrm{H}$ and Kashiwakura I (2012) Functional properties of synthetic Nacyl-L-homoserine lactone analogs of quorum-sensing gramnegative bacteria on the growth of human oral squamous carcinoma cells. Invest New Drugs 30:157-163

141. Soejima K, Fang W and Rollins BJ (2003) DNA methyltransferase 3b contributes to oncogenic transformation induced by SV40T antigen and activated Ras. Oncogene 22:4723-4733

142. Serrano M, Lin AW, McCurrach ME, Beach D and Lowe SW (1997) Oncogenic ras provokes premature cell senescence associated with accumulation of p53 and p16INK4a. Cell 88:593-602 
143. Azua J, Romeo P, Serrano M, Martinez TD and Azua J, Jr. (1997) Prognostic value from DNA quantification by static cytometry in breast cancer. Anal. Quant. Cytol. Histol. 19:80-86

144. Hahn WC, Counter CM, Lundberg AS, Beijersbergen RL, Brooks MW and Weinberg RA (1999) Creation of human tumour cells with defined genetic elements. Nature 400:464-468

145. Kelly PN and Strasser A (2011) The role of Bcl-2 and its pro-survival relatives in tumourigenesis and cancer therapy. Cell Death. Differ. 18:1414-1424

146. Huang H, Shah K, Bradbury NA, Li C and White C (2014) Mcl-1 promotes lung cancer cell migration by directly interacting with VDAC to increase mitochondrial $\mathrm{Ca} 2+$ uptake and reactive oxygen species generation. Cell Death. Dis. 5:e1482

147. Vaux DL, Cory S and Adams JM (1988) Bcl-2 gene promotes haemopoietic cell survival and cooperates with c-myc to immortalize pre-B cells. Nature 335:440-442

148. Renault TT and Chipuk JE (2014) Death upon a kiss: mitochondrial outer membrane composition and organelle communication govern sensitivity to BAK/BAX-dependent apoptosis. Chem. Biol. 21:114123

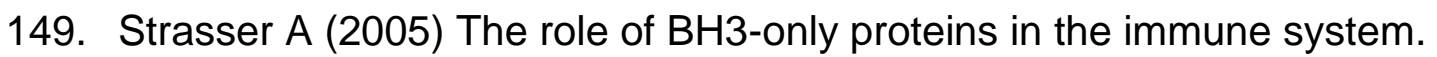
Nat. Rev. Immunol. 5:189-200

150. Eno CO, Zhao G, Venkatanarayan A, Wang B, Flores ER and Li C (2013) Noxa couples lysosomal membrane permeabilization and apoptosis during oxidative stress. Free Radic. Biol. Med. 65:26-37

151. Yu J, Wang Z, Kinzler KW, Vogelstein B and Zhang L (2003) PUMA mediates the apoptotic response to p53 in colorectal cancer cells. Proc. Natl. Acad. Sci. U. S. A 100:1931-1936

152. Straten $\mathrm{P}$ and Andersen $\mathrm{MH}$ (2010) The anti-apoptotic members of the Bcl-2 family are attractive tumor-associated antigens. Oncotarget. $1: 239-245$

153. Vogler M, Dinsdale D, Dyer MJ and Cohen GM (2009) Bcl-2 inhibitors: small molecules with a big impact on cancer therapy. Cell Death. Differ. 16:360-367

154. Mullauer FB, Kessler JH and Medema JP (2009) Betulinic acid induces cytochrome $\mathrm{c}$ release and apoptosis in a Bax/Bak-independent, 
permeability transition pore dependent fashion. Apoptosis. 14:191202

155. Wan KF, Chan SL, Sukumaran SK, Lee MC and Yu VC (2008)

Chelerythrine induces apoptosis through a Bax/Bak-independent mitochondrial mechanism. J. Biol. Chem. 283:8423-8433

156. Leanza L, Henry B, Sassi N, Zoratti M, Chandy KG, Gulbins E and Szabo I (2012) Inhibitors of mitochondrial Kv1.3 channels induce Bax/Bakindependent death of cancer cells. EMBO Mol. Med. 4:577-593

157. Zhu Y, Eaton JW and Li C (2012) Titanium dioxide (TiO2) nanoparticles preferentially induce cell death in transformed cells in a Bak/Baxindependent fashion. PLoS. One. 7:e50607

158. Lei X, Chen Y, Du G, Yu W, Wang X, Qu H, Xia B, He H, Mao J, Zong W, Liao X, Mehrpour M, Hao X and Chen Q (2006) Gossypol induces Bax/Bak-independent activation of apoptosis and cytochrome $\mathrm{C}$ release via a conformational change in Bcl-2. FASEB J. 20:21472149

159. Harel M, Aharoni A, Gaidukov L, Brumshtein B, Khersonsky O, Meged R, Dvir H, Ravelli RB, McCarthy A, Toker L, Silman I, Sussman JL and Tawfik DS (2004) Structure and evolution of the serum paraoxonase family of detoxifying and anti-atherosclerotic enzymes. Nat. Struct. Mol. Biol. 11:412-419

160. Hanahan D and Weinberg RA (2011) Hallmarks of cancer: the next generation. Cell 144:646-674

161. Ross ME, Zhou X, Song G, Shurtleff SA, Girtman K, Williams WK, Liu HC, Mahfouz R, Raimondi SC, Lenny N, Patel A and Downing JR (2003) Classification of pediatric acute lymphoblastic leukemia by gene expression profiling. Blood 102:2951-2959

162. Ribarska T, Ingenwerth M, Goering W, Engers R and Schulz WA (2010) Epigenetic inactivation of the placentally imprinted tumor suppressor gene TFPI2 in prostate carcinoma. Cancer Genomics Proteomics. 7:51-60

163. Li Y, Li Y, Tang R, Xu H, Qiu M, Chen Q, Chen J, Fu Z, Ying K, Xie Y and Mao $Y$ (2002) Discovery and analysis of hepatocellular carcinoma genes using cDNA microarrays. J. Cancer Res. Clin. Oncol. 128:369-379

164. Frank O, Brors B, Fabarius A, Li L, Haak M, Merk S, Schwindel U, Zheng C, Muller MC, Gretz N, Hehlmann R, Hochhaus A and Seifarth W 
(2006) Gene expression signature of primary imatinib-resistant chronic myeloid leukemia patients. Leukemia 20:1400-1407

165. Shih DM and Lusis AJ (2009) The roles of PON1 and PON2 in cardiovascular disease and innate immunity. Curr. Opin. Lipidol. 20:288-292

166. Rajkovic MG, Rumora $L$ and Barisic $K$ (2011) The paraoxonase 1,2 and 3 in humans. Biochem. Med. (Zagreb. ) 21:122-130

167. Kowalska K, Socha E and Milnerowicz H (2015) Review: The role of paraoxonase in cardiovascular diseases. Ann. Clin. Lab Sci. 45:226-233

168. Primo-Parmo SL, Sorenson RC, Teiber J and La Du BN (1996) The human serum paraoxonase/arylesterase gene (PON1) is one member of a multigene family. Genomics 33:498-507

169. La Du BN (1996) Structural and functional diversity of paraoxonases. Nat. Med. 2:1186-1187

170. Mackness MI, Arrol S and Durrington PN (1991) Paraoxonase prevents accumulation of lipoperoxides in low-density lipoprotein. FEBS Lett. 286:152-154

171. Reddy ST, Wadleigh DJ, Grijalva V, Ng C, Hama S, Gangopadhyay A, Shih DM, Lusis AJ, Navab M and Fogelman AM (2001) Human paraoxonase-3 is an HDL-associated enzyme with biological activity similar to paraoxonase-1 protein but is not regulated by oxidized lipids. Arterioscler. Thromb. Vasc. Biol. 21:542-547

172. Ng CJ, Wadleigh DJ, Gangopadhyay A, Hama S, Grijalva VR, Navab M, Fogelman AM and Reddy ST (2001) Paraoxonase-2 is a ubiquitously expressed protein with antioxidant properties and is capable of preventing cell-mediated oxidative modification of low density lipoprotein. J. Biol. Chem. 276:44444-44449

173. Mochizuki H, Scherer SW, Xi T, Nickle DC, Majer M, Huizenga JJ, Tsui LC and Prochazka M (1998) Human PON2 gene at 7q21.3: cloning, multiple mRNA forms, and missense polymorphisms in the coding sequence. Gene 213:149-157

174. Witte I, Foerstermann U, Devarajan A, Reddy ST and Horke S (2012) Protectors or Traitors: The Roles of PON2 and PON3 in Atherosclerosis and Cancer. J. Lipids 2012:342806 
175. Devarajan A, Shih D and Reddy ST (2014) Inflammation, infection, cancer and all that...the role of paraoxonases. Adv. Exp. Med. Biol. 824:3341

176. Fuqua C, Parsek MR and Greenberg EP (2001) Regulation of gene expression by cell-to-cell communication: acyl-homoserine lactone quorum sensing. Annu. Rev. Genet. 35:439-468

177. Bar-Rogovsky H, Hugenmatter A and Tawfik DS (2013) The evolutionary origins of detoxifying enzymes: the mammalian serum paraoxonases (PONs) relate to bacterial homoserine lactonases. J. Biol. Chem. 288:23914-23927

178. Teiber JF, Horke S, Haines DC, Chowdhary PK, Xiao J, Kramer GL, Haley RW and Draganov DI (2008) Dominant role of paraoxonases in inactivation of the Pseudomonas aeruginosa quorum-sensing signal N-(3-oxododecanoyl)-L-homoserine lactone. Infect. Immun. 76:2512-2519

179. Altenhofer S, Witte I, Teiber JF, Wilgenbus P, Pautz A, Li H, Daiber A, Witan $\mathrm{H}$, Clement AM, Forstermann U and Horke S (2010) One enzyme, two functions: PON2 prevents mitochondrial superoxide formation and apoptosis independent from its lactonase activity. J. Biol. Chem. 285:24398-24403

180. Delgado O, Kaisani AA, Spinola M, Xie XJ, Batten KG, Minna JD, Wright WE and Shay JW (2011) Multipotent capacity of immortalized human bronchial epithelial cells. PLoS. One. 6:e22023

181. Devarajan A, Grijalva VR, Bourquard N, Meriwether D, III, Imaizumi S, Shin BC, Devaskar SU and Reddy ST (2012) Macrophage paraoxonase 2 regulates calcium homeostasis and cell survival under endoplasmic reticulum stress conditions and is sufficient to prevent the development of aggravated atherosclerosis in paraoxonase 2 deficiency/apoE-/- mice on a Western diet. Mol. Genet. Metab 107:416-427

182. Rosenblat M, Draganov D, Watson CE, Bisgaier CL, La Du BN and Aviram M (2003) Mouse macrophage paraoxonase 2 activity is increased whereas cellular paraoxonase 3 activity is decreased under oxidative stress. Arterioscler. Thromb. Vasc. Biol. 23:468-474

183. Horke $S$, Witte I, Wilgenbus $P$, Kruger M, Strand $D$ and Forstermann $U$ (2007) Paraoxonase-2 reduces oxidative stress in vascular cells and decreases endoplasmic reticulum stress-induced caspase activation. Circulation 115:2055-2064 
184. Shiner M, Fuhrman B and Aviram M (2004) Paraoxonase 2 (PON2) expression is upregulated via a reduced-nicotinamide-adeninedinucleotide-phosphate (NADPH)-oxidase-dependent mechanism during monocytes differentiation into macrophages. Free Radic. Biol. Med. 37:2052-2063

185. Bourquard N, Ng CJ and Reddy ST (2011) Impaired hepatic insulin signalling in PON2-deficient mice: a novel role for the PON2/apoE axis on the macrophage inflammatory response. Biochem. J. 436:91-100

186. Droge W (2002) Free radicals in the physiological control of cell function. Physiol Rev. 82:47-95

187. Storz $\mathrm{P}$ (2005) Reactive oxygen species in tumor progression. Front Biosci. 10:1881-1896

188. Liou GY and Storz P (2010) Reactive oxygen species in cancer. Free Radic. Res. 44:479-496

189. Gomes M, Teixeira AL, Coelho A, Araujo A and Medeiros R (2014) The role of inflammation in lung cancer. Adv. Exp. Med. Biol. 816:1-23

190. WARBURG O (1956) On the origin of cancer cells. Science 123:309-314

191. Gottlieb E and Tomlinson IP (2005) Mitochondrial tumour suppressors: a genetic and biochemical update. Nat. Rev. Cancer 5:857-866

192. Moreno-Sanchez R, Rodriguez-Enriquez S, Marin-Hernandez A and Saavedra E (2007) Energy metabolism in tumor cells. FEBS J. 274:1393-1418

193. Funes JM, Quintero M, Henderson S, Martinez D, Qureshi U, Westwood C, Clements MO, Bourboulia D, Pedley RB, Moncada S and Boshoff C (2007) Transformation of human mesenchymal stem cells increases their dependency on oxidative phosphorylation for energy production. Proc. Natl. Acad. Sci. U. S. A 104:6223-6228

194. Telang S, Nelson KK, Siow DL, Yalcin A, Thornburg JM, ImbertFernandez Y, Klarer AC, Farghaly H, Clem BF, Eaton JW and Chesney $\mathrm{J}$ (2012) Cytochrome c oxidase is activated by the oncoprotein Ras and is required for A549 lung adenocarcinoma growth. Mol. Cancer 11:60

195. Weinberg F, Hamanaka R, Wheaton WW, Weinberg S, Joseph J, Lopez M, Kalyanaraman B, Mutlu GM, Budinger GR and Chandel NS (2010) Mitochondrial metabolism and ROS generation are essential 
for Kras-mediated tumorigenicity. Proc. Natl. Acad. Sci. U. S. A 107:8788-8793

196. Ng CJ, Bourquard N, Grijalva V, Hama S, Shih DM, Navab M, Fogelman AM, Lusis AJ, Young S and Reddy ST (2006) Paraoxonase-2 deficiency aggravates atherosclerosis in mice despite lower apolipoprotein-B-containing lipoproteins: anti-atherogenic role for paraoxonase-2. J. Biol. Chem. 281:29491-29500 


\section{APPENDIX: LIST OF ABBREVIATIONS}

$\begin{array}{ll}\text { 5-FU } & \text { 5-Fluorouracil } \\ \text { Apaf-1 } & \text { Apoptotic Protease Factor 1 } \\ \text { BH } & \text { Bcl-2 homolog } \\ \text { C12 } & \text { N-(3-oxododecanoyl)-homoserine lactone } \\ \text { COQ10 } & \text { Coenzyme Q10 } \\ \text { DISC } & \text { Death Inducing Signaling Complex } \\ \text { FADD } & \text { Fas Associated Death Domain } \\ \text { FasL } & \text { Fas Ligand } \\ \text { FBS } & \text { Fetal Bovine Serum } \\ \text { IAPs } & \text { Inhibitor of Apoptosis Proteins } \\ \text { LLC } & \text { Lewis Lung Carcinoma } \\ \text { MEFs } & \text { Mouse Embryonic Fibroblasts } \\ \text { MIPs } & \text { Maximum Intensity Projections } \\ \text { MOMP } & \text { Nonondrial Outer Membrane Permeabilization } \\ \text { NHE } & \end{array}$




$\begin{array}{ll}\text { OMM } & \text { Outer Mitochondrial Membrane } \\ \text { PBS } & \text { Phosphate Buffered Saline } \\ \text { PI } & \text { Propidium lodide } \\ \text { PON2 } & \text { Paraoxonase } 2 \\ \text { ROIS } & \text { Regions of Interest } \\ \text { TLR4 } & \text { Toll-Like Receptor 4 } \\ \text { TNF } & \text { Tumor Necrosis Factor } \\ \text { TNFR } & \text { Tumor Necrosis Factor Receptor } \\ \text { TNFR1 } & \text { Tumor Necrosis Factor Receptor 1 } \\ \text { TRAIL } & \text { TNF-Related Apoptosis Inducing Ligand } \\ \text { WT } & \text { Wild Type }\end{array}$




\section{CURRICULUM VITAE}

\section{Aaron Mackallan Neely}

PRESENT ADDRESS:

TELEPHONE:

EMAIL:

PLACE OF BIRTH:

DATE OF BIRTH:

VISA STATUS:
Department of Pharmacology and Toxicology, School of Medicine, University of Louisville, 505 S Hancock St. Rm 433A Louisville, KY 40202

(469)682-5909

aaron neely@hotmail.com;

amneel04@louisville.edu

Nassau, Bahamas

April 25 1983

F1 Visa

\section{EDUCATION:}

08/2013-present: Ph.D., Department of Pharmacology and Toxicology, University of Louisville, Louisville, Kentucky

08/2013-03/2016: M.S., Pharmacology and Toxicology, University of Louisville, Louisville, Kentucky

08/2009-08/2012: M.S., Biology (Developmental Physiology), University of North Texas, Denton, Texas

8/2003-05/2007: B.S., Biology, Paul Quinn College, Dallas, Texas

PROFESSIONAL EXPERIENCE:

08/2013-present: Graduate Research assistant, Department of Pharmacology and Toxicology, University of Louisville, Louisville, Kentucky (Advisor Dr. Chi Li)

02/2012-08/2013: Recovery Analyst, Real Time Resolutions- Chase Department Dallas, Texas (Supervisor Jordan Schnieder)

06/2011-08/2012: Teacher's Assistant/Lab Instructor-Introduction to Biology I, University of North Texas, Denton, Texas

01/2011-05/2011 Teacher's Assistant/Lab Instructor-Animal Physiology, University of North Texas, Denton, Texas

01/2010-12/2010 Teacher's Assistant/Lab Instructor- Human Anatomy \& 


\begin{abstract}
Physiology I, University of North Texas, Denton, Texas
01/2009-12/2009 Teacher's Assistant/Lab Instructor- Human Anatomy and Physiology II, University of North Texas, Denton, Texas

01/2009- 05/2012: Graduate Research assistant, Department of Biology, University of North Texas, Denton, Texas (Advisor Edward Dzialowski)

09/2001-1

2/2008:

Customer Service Representative/ Personal Banker, the Royal Bank of Canada, Nassau, Bahamas (Supervisor Natasha, Huyler)

07/2000-08/2001: Office Clerk, The Private Trust Corporation (PTC), Nassau, Bahamas (Supervisor Kenya Pickersgill)
\end{abstract}

\title{
PROFESSIONAL MEMBERSHIPS:
}

2016-present: American Society for Pharmacology and Experimental Therapeutics

2016-present: $\quad$ American Society of Cell Biology

2016-present: American Society of Clinical Oncology

2016-present: $\quad$ Society of Toxicology

2016-present: Ohio Valley Society of Toxicology

2004-present: $\quad$ Phi Eta Sigma Honor Society

\section{MAJOR AWARDS AND SCHOLARSHIPS:}

04/2017:

12/2016:

The University of Louisville Graduate Student Council Travel Scholarship The American Society of Cell Biology Annual meeting Travel Award

10/2016:

The Ohio Valley Society of Toxicology 2016 Annual Meeting-

2013-2015: $\quad$ University of Louisville Integrated Program in Biomedical Sciences (IPIBS) Doctoral Fellowship

2009-2012: $\quad$ University of North Texas Physiology Graduate Assistantship, Denton, Texas University of North Texas Biology Teacher's Assistantship, Denton, Texas

The Good Neighbor Scholarship, Denton, Texas

2003-2007: International Student Scholarship, Paul Quinn College, Dallas, Texas

Track and Field Scholarship, Paul Quinn College, Dallas Texas 


\section{PUBLICATIONS:}

Aaron M. Neely, Guoping Zhao, Christian Schwarzer, Nicole S. Stivers, Aaron G. Whitt, Joseph A. Burlison, Terry E. Machen and Chi Li. N-(3-oxo-acyl)-homoserine lactone induces apoptosis exclusively through a mitochondrial pathway (submitted to Scientific Reports)

Guoping Zhao, Aaron M. Neely, Christian Schwarzer, Huayi Lu, Aaron G. Whitt, Joseph A. Burlison, Terry E. Machen and Chi Li (2016) N-(3-oxoacyl)homoserine lactone inhibits tumor growth independent of $\mathrm{Bcl}-2$ proteins. Oncotarget. 7(5): 5924-5942.

Christian Schwarzer, Zhu Fu, Takeshi Morita, Aaron G. Whitt, Aaron M. Neely, Chi Li and Terry E. Machen. (2015) Paraoxonase 2 mediates $\mathrm{N}$-(3oxododecanoyl)-homoserine lactone-triggered apoptosis in fibroblasts. Journal of Biological Chemistry. 290:8949-8963.

\section{ABSTRACTS SUBMITTED TO REGIONAL AND NATIONAL MEETINGS:}

Aaron M. Neely, Guoping Zhao, Yanglong Zhu Colins O. Eno, Yanlong, Lynn DeLeeuw, Joseph A. Burlison, Jonathan B. Chaires, John O. Trent, and Chi Li. Inducing tumor cell apoptosis by a small molecule activating Bax. The American Society of Cell Biology Annual Meeting December 2016

Neely, A.M., Zhao, G., Whitt A., Chi L., Scwarzer C., Huayi, L., Stivers, N., Burlison, J., Machen, T. and Chi Li. The bacterial quorum sensing molecule N-(3oxo-acyl) homoserine lactone inhibits tumor growth independent of Bcl-2 proteins. The Ohio Valley Society of Toxicology Meeting October 2016

Neely, A.M., Zhao, G., Whitt A., Chi L., Scwarzer C., Huayi, L., Stivers, N., Burlison, J., Machen, T. and Chi Li. The bacterial quorum sensing molecule N-(3oxo-acyl) homoserine lactone inhibits tumor growth independent of Bcl-2 proteins. Kentucky Lung Cancer Symposium October 2016

Neely, A.M., Zhao, G., Whitt A., Chi L., Scwarzer C., Huayi, L., Stivers, N., Burlison, J., Machen, T. and Chi Li. The bacterial quorum sensing molecule N-(3oxo-acyl) homoserine lactone inhibits tumor growth independent of Bcl-2 proteins. Research! Louisville October 2016

Neely, A.M., Zhao, G., Whitt A., Chi L., Scwarzer C., Huayi, L., Stivers, N., Burlison, J., Machen, T. and Chi Li. The bacterial quorum sensing molecule N-(3oxo-acyl) homoserine lactone inhibits tumor growth independent of $\mathrm{Bcl}-2$ 
proteins. Ohio Valley Society of Toxicology Student/PostDoc Summer Meeting June 2016

Neely, A.M., Zhao, G., Whitt Aaron, Chi Li, Distinct roles of paraoxonase 2 in apoptosis signaling cell proliferation. Research! Louisville September 2015

Neely, A.M., Zhao, G., Stivers, N.S., and Li, C. The Psuedomonas Aeruginosa Quorum-sensing Molecule Homoserine Lactone Triggers a Unique Mitochondrial Apoptotic Signaling Pathway. Ohio Valley Society of Toxicology Student/PostDoc Summer Meeting June 2015

Neely, A.M., Zhao, G., Stivers, N.S., and Li, C. The Psuedomonas Aeruginosa Quorum-sensing Molecule Homoserine Lactone Triggers a Unique Mitochondrial Apoptotic Signaling Pathway. University of Louisville Graduate Student Research Symposium November 2014.

Neely, A.M., Zhao, G., Stivers, N.S., and Li, C. The Psuedomonas Aeruginosa Quorum-sensing Molecule Homoserine Lactone Triggers a Unique Mitochondrial Apoptotic Signaling Pathway. James Graham Brown Cancer Retreat November 2014.

Neely, A.M., Zhao, G., Stivers, N.S., and Li, C. The Psuedomonas Aeruginosa Quorum-sensing Molecule Homoserine Lactone Triggers a Unique Mitochondrial Apoptotic Signaling Pathway. Research! Louisville October 2014.

\section{TECHNICAL SKILLS:}

\section{CELL AND MOLECULAR BIOLOGY TECHNIQUES:}

Crispr/Cas9, Enzyme Linked Immunosorbent Assay (ELISA), Biochemical Fractionation, Spectrophotometry, Proteomics, Polymerase chain reaction (PCR), reverse transcriptase PCR (rtPCR), quantitative real time PCR (qPCR), molecular cloning, mitochondrial purification, western blot analysis, flow cytometry analysis, immunoprecipitation, immunofluorescence analysis, Terminal Uridine Nick-End Labeling (TUNEL), retroviral infection, microscopy, cell culture.

\section{ANIMAL HANDLING TECHNIQUES:}

Humane handling and restraint 
Measurement of Metabolic Rates (Oxygen Consumption): Chicken (Gallus gallus), Alligator and Muscovy ducks

Mice: Blood collection, Anesthesia administration, Euthanasia, Injections, Tumor Transplantation

\section{COMPUTER SKILLS:}

Microsoft office (MS word, MS Excel, MS power point), Adobe Photoshop,

Canvas, EndNote 9, Reference Manager. 